Aus der Abteilung Allgemeinchirurgie

(Prof. Dr. med. H. Becker)

Im Zentrum Chirurgie

der Medizinischen Fakultät der Universität Göttingen

\title{
Therapie breitbasiger Adenome und der pT1-low-risk-Karzinome des Rektums
}

Ein Vergleich der radikalchirurgischen Verfahren mit der konventionellen Abtragung und der transanalen endoskopischen Mikrochirurgie

\author{
INAUGURAL-DISSERTATION \\ zur Erlangung des Doktorgrades \\ der Medizinischen Fakultät \\ der Georg-August-Universität zu Göttingen
}

vorgelegt von

Malte Süß

aus

Oldenburg

Göttingen 2005 
Dekan: $\quad$ Prof. Dr. med. W. Brück

I. Berichterstatter: $\quad$ PD Dr. med. C. Langer

II. Berichterstatter/in: Prof. Dr. med. G. Ramadori

III. Berichterstatter/in:

Tag der mündlichen Prüfung: 30. Mai 2005 


\section{Inhaltsverzeichnis}

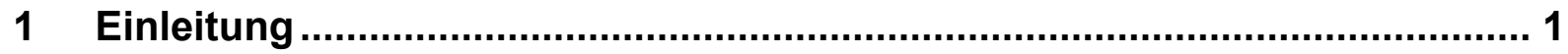

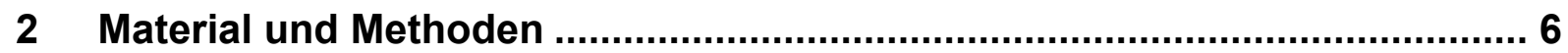

2.1 Zusammensetzung des Patientenkollektivs ………............................. 6

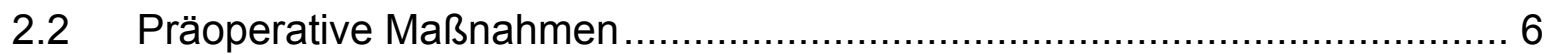

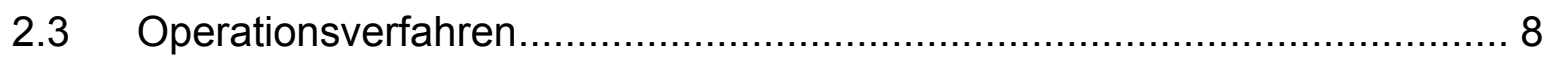

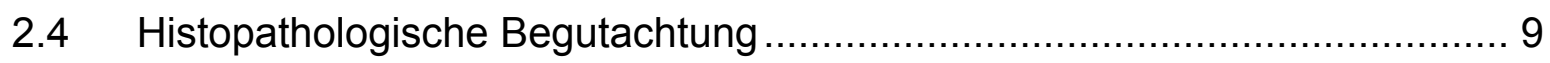

2.5 Nachuntersuchung und Follow up ...................................................... 10

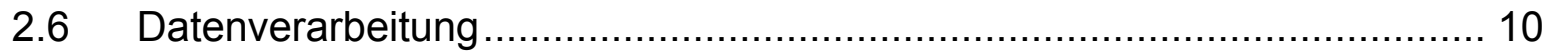

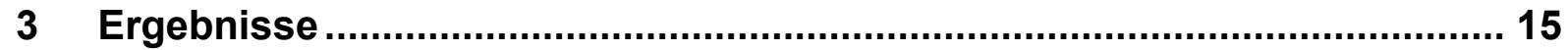

3.1 Zusammensetzung des Kollektivs ................................................ 15

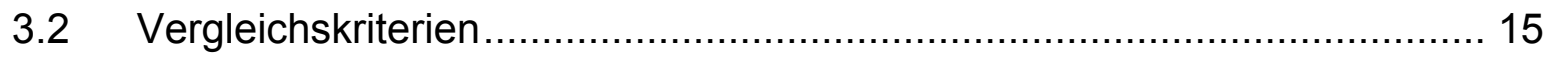

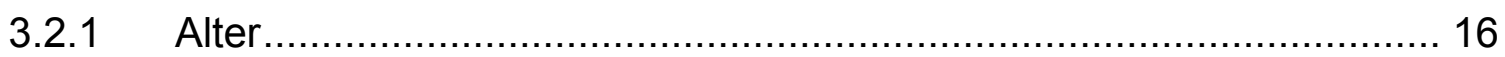

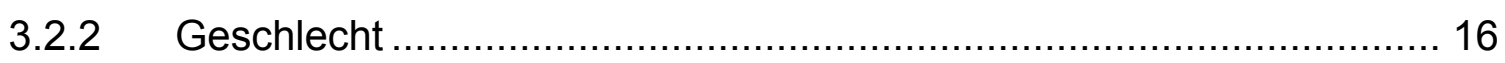

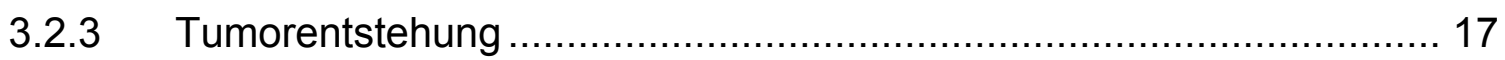

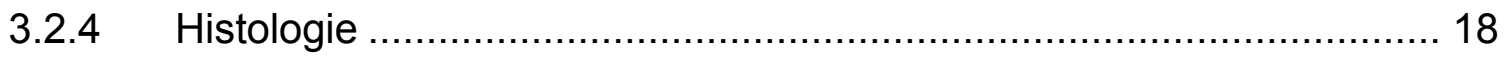

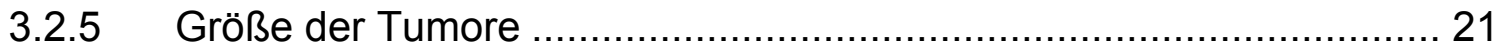

3.2.6 Höhenlokalisation der Tumore ab ano ………............................ 22

3.2.7 Tumorlokalisation bezogen auf die Zirkumferenz des Rektums.......... 23

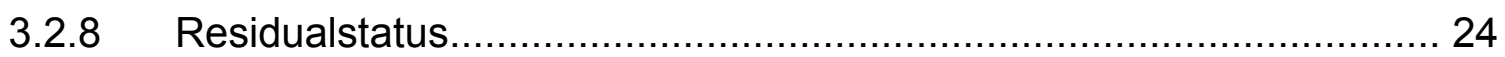

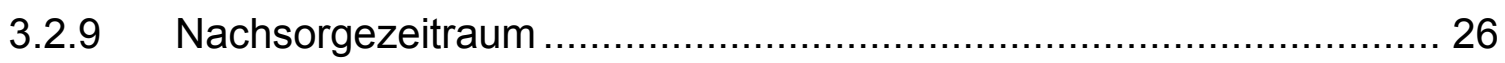

3.2.10 Zusammenfassung der Vergleichskriterien .................................... 27

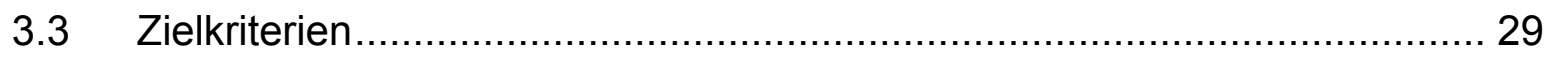

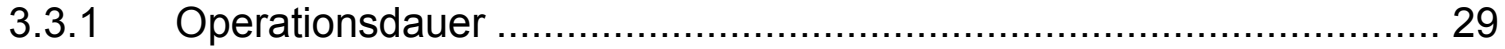

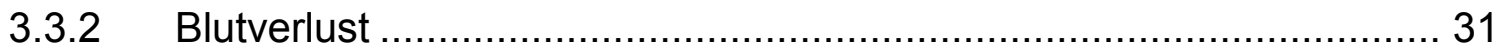

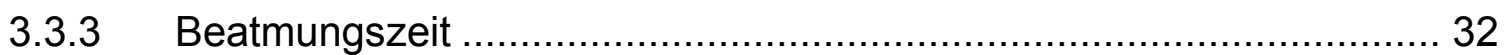

3.3.4 Postoperativer stationärer Aufenthalt ............................................ 33

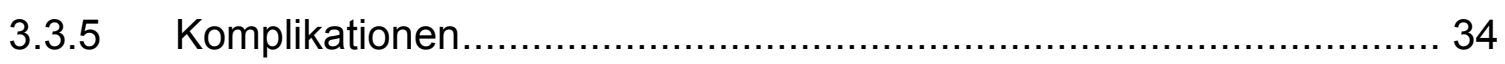

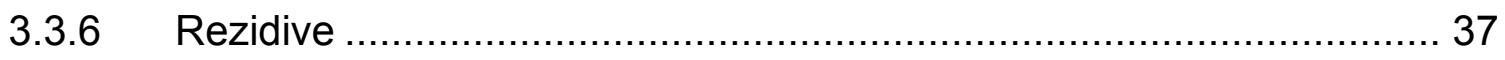

3.2.7 Zusammenfassung der Zielkriterien ........................................... 41

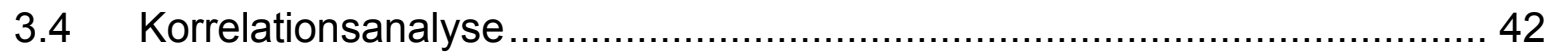

3.4.1 Einflussfaktoren auf die Komplikationsrate .................................. 42

3.4.2 Einflussfaktoren auf den Residualstatus .......................................... 43

3.4.3 Einflussfaktoren auf die Rezidivrate ............................................. 44

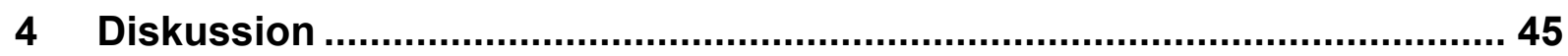

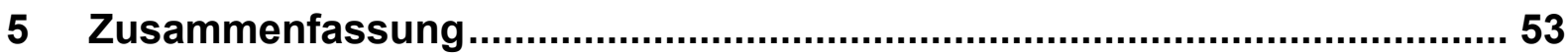

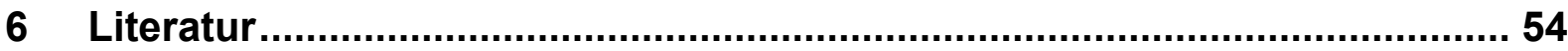




\section{Verzeichnis der Tabellen}

Tabelle 1: Kriterien lokaler Resektabilität …………......................................... 7

Tabelle 2: Nachsorgeschema ..................................................................... 10

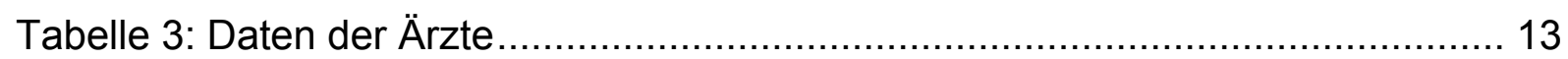

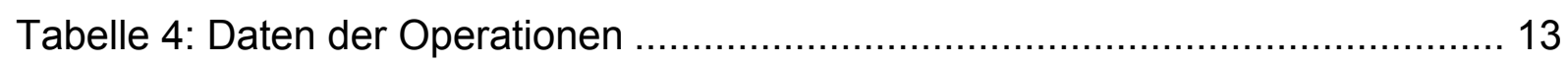

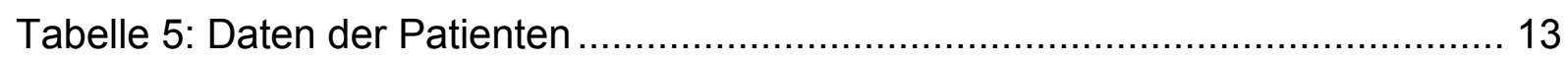

Tabelle 6: Daten der Komplikationen.............................................................. 13

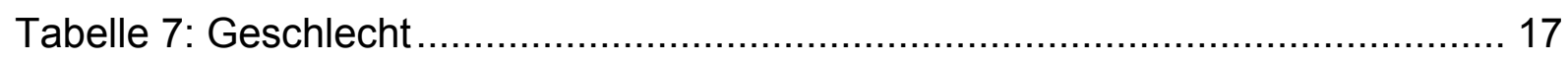

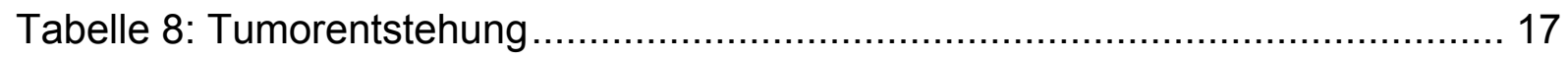

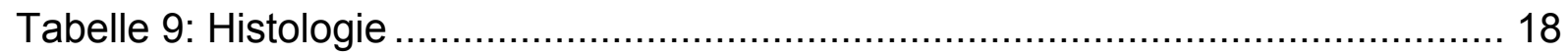

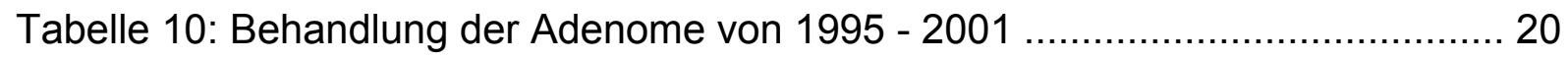

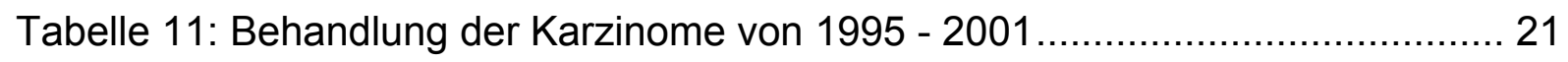

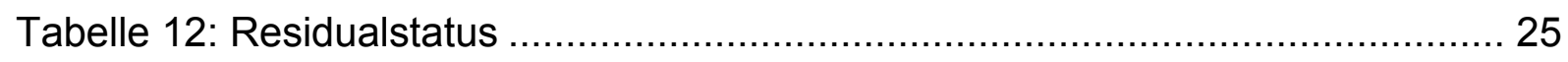

Tabelle 13: Zusammenfassung der Vergleichskriterien ........................................ 28

Tabelle 14: Bedarf an Erythrozytenkonzentraten................................................... 31

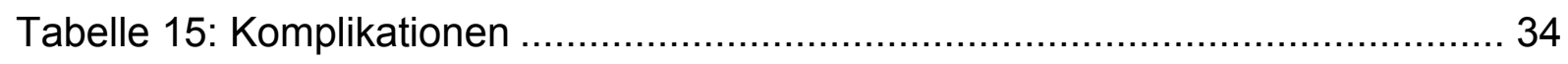

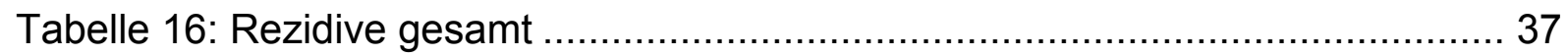

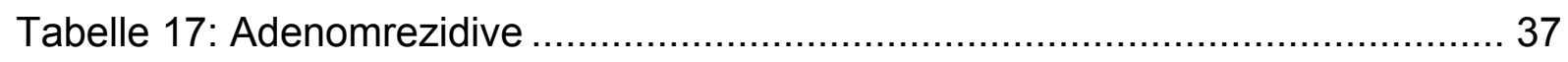

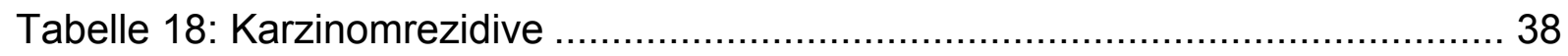

Tabelle 19: Zusammenfassung der Zielkriterien ............................................... 42

Tabelle 20: Einflussfaktoren auf die Komplikationsrate ....................................... 43

Tabelle 21: Einflussfaktoren auf den Residualstatus .......................................... 43

Tabelle 22: Einflussfaktoren auf die Rezidivrate .................................................. 44 


\section{Verzeichnis der Abbildungen}

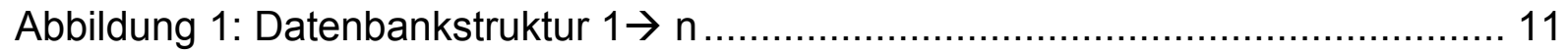

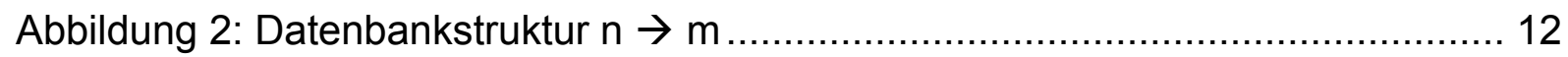

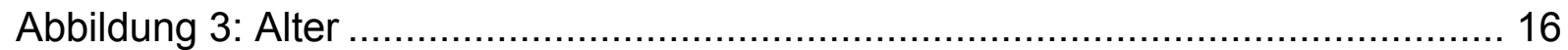

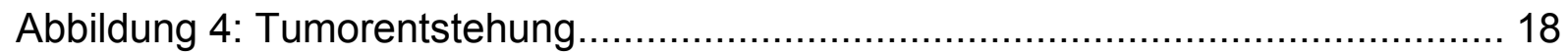

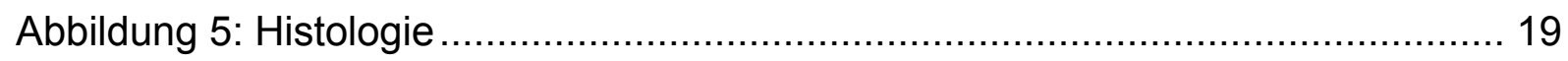

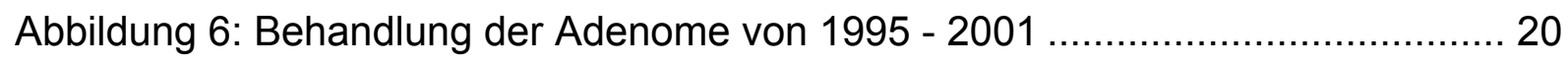

Abbildung 7: Behandlung der Karzinome von 1995 - 2001 ................................. 21

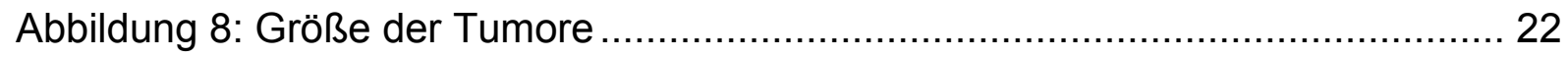

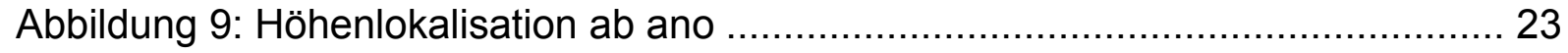

Abbildung 10: Tumorlokalisation bezogen auf die Zirkumferenz des Rektums........ 24

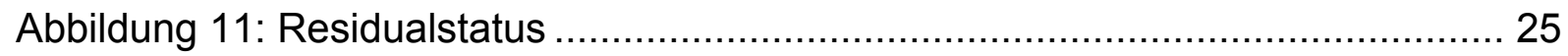

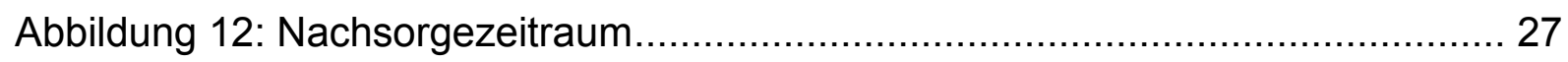

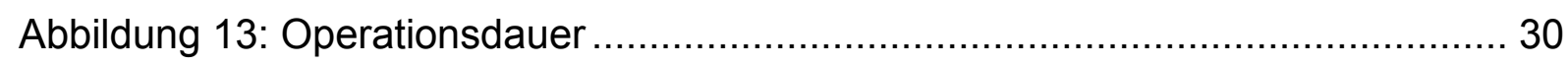

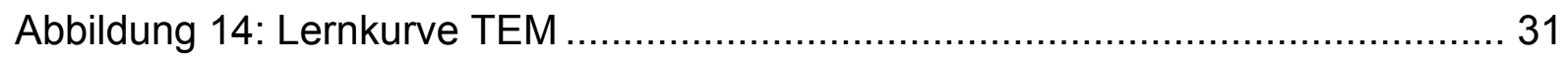

Abbildung 15: Bedarf an Erythrozytenkonzentraten .................................. 32

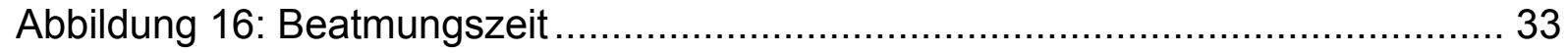

Abbildung 17: Postoperativer stationärer Aufenthalt........................................ 34

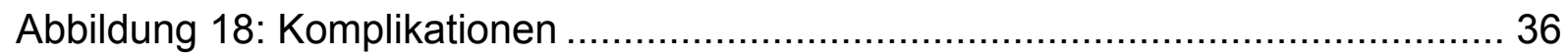

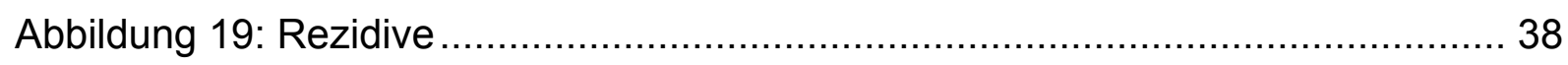

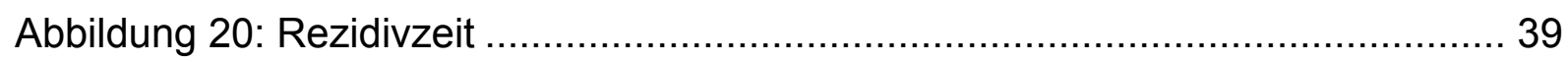




\section{$1 \quad$ Einleitung}

Tumore des Rektums zählen in den industrialisierten Staaten zu den häufigsten neoplastischen Veränderungen des Seniums (Nöthinger et Birrer 1999, Robert-KochInstitut 1999). Für das Rektumkarzinom wird im Gesundheitsbericht der Bundesrepublik Deutschland eine Inzidenz von 37/100.000/Jahr angegeben, was es zum zweithäufigsten Karzinom der Frau nach dem Brustkrebs und dem zweithäufigsten des Mannes nach dem Bronchialkarzinom macht (Schön et al. 1999). Tatsächlich liegt die Anzahl Erkrankter jedoch wesentlich höher. Die größte hierzu durchgeführte Untersuchung (Arminski et McLeand 1964) zeigte bei Autopsien an 1000 asymptomatischen Patienten in der Altersgruppe zwischen 70 und 79 Jahren in 8,3\% neoplastische Veränderungen des Rektums. Aufgrund intensivierter Vorsorgemaßnahmen und deren zunehmender Akzeptanz ist daher zukünftig mit einer steigenden Inzidenz (Mainprize et al. 1998, Midgley et Kerr. 1999), insbesondere der frühen Tumorstadien zu rechnen.

Die häufigsten Tumore des Rektums sind Adenome und Karzinome, die man nach unterschiedlichen histopathologischen Kriterien beurteilt. Adenome werden dem Wachstumsmuster nach in tubuläre, villöse und eine tubulovillöse Zwischenform unterschieden sowie dem Differenzierungsgrad folgend den Stadien 1 bis 4 zugeordnet. Zur Festlegung des Stadiums der Tumorerkrankung für Karzinome wird die sogenannte TNM-Klassifikation (Hermanek et al. 1999) verwendet, die nach der Infiltrationstiefe $(\mathrm{pT})$, dem Befall von lokoregionären Lymphknoten $(\mathrm{pN})$ sowie Fernmetastasen (pM) unterscheidet. Eine Sonderform des Karzinoms bildet hierbei das sogenannte Carcinoma in situ (Tis), das gemäß der Definition für Karzinome infiltrativ wächst, die muköse Basalmembran jedoch noch nicht überschritten hat, und damit per definitionem keine Lymphknotenmetastasierung zeigt.

Der Pathomechanismus, der zur Entstehung des Rektumkarzinoms führt, wird heute als so genannter genetic multistep process betrachtet (Boland 1993), der ohne Kenntnis der genetischen Hintergründe bereits in den achtziger Jahren postuliert wurde (Muto et al. 1975). Laut dieser nach Vogelstein und Kinzler benannten Hypothese (Vogelstein et Kinzler 2002) entwickelt sich ein Karzinom im Rahmen einer Adenom-Karzinom-Sequenz aus gesunder Schleimhaut. Initial kommt es dieser Theorie entsprechend auf der Grundlage einer Mutation des sogenannten 
Adenomatöse-Polyposis-coli (APC)-Gens zur Dysplasie der Schleimhaut. Im Verlauf weiterer Mutationen entwickelt sich hieraus ein Adenom. Der Verlust des Gens p53 in einem der Zellklone eines solchen Adenoms führt konsekutiv zu unkontrolliertem invasiven Wachstum und damit zum Karzinom. Das Zeitintervall zwischen der Entstehung eines Adenoms und dessen weitergehender Entartung zu einem Karzinom kann für den Menschen nicht exakt bestimmt werden. Schätzungen nach bedarf dieser Prozess zwischen 10 und 15 Jahren (Muto et al. 1975). Die Geschwindigkeit, mit der dieser Prozess fortschreitet, scheint von mehreren Faktoren beeinflusst $\mathrm{zu}$ werden. So konnte ein signifikanter Zusammenhang des Fortschreitens der Entdifferenzierung sowohl mit der Größe der Adenome als auch deren villöser Komponente gezeigt werden (Jørgensen et al. 1993). Die Hypothese eines kontinuierlichen Übergangs vom Adenom zum Karzinom und dessen Abhängigkeit von der Größe der Adenome wird auch durch autoptische Daten untermauert. So zeigten von 797 Polypen solche mit weniger als $1 \mathrm{~cm}$ Durchmesser keine maligne Transformation. In Adenomen von mehr als $3 \mathrm{~cm}$ Größe konnte jedoch bereits in $30 \%$ der Fälle eine karzinomatöse Entartung nachgewiesen werden (O'Brien et al. 1990, Mörschel et al. 1999).

Rektumtumore stellen aufgrund ihrer Häufigkeit und der damit verbundenen sozioökonomischen Bedeutung (Midgley et Kerr 1999), aber auch aufgrund der schlechten Zugänglichkeit und des potentiell letalen Krankheitsverlaufs ein Problemfeld chirurgischer Bemühungen dar (Kasperk et al. 2001). Als kurativer Ansatz zur Therapie von Tumoren des Enddarms stand zunächst nur die radikale Entfernung des Rektums zur Verfügung. Eine der ersten dokumentierten Operationen wird Faget zugeschrieben, der im Jahr 1739 einen Rektumtumor perineal reseziert haben soll; im Jahr 1826 veröffentlichte Lisfranc seine Erfahrungen mit 9 perinealen Tumorresektionen (Lisfranc 1826). Mit wachsendem Verständnis der Bedeutung lymphatischer Metastasierung des Rektumkarzinoms für die Prognose der Patienten wurde durch Czerny (1880) und Miles (1908) die abdominoperineale Rektumexstirpation unter Mitnahme des lokoregionären Lymphgewebes des Rektums, dem sogenannten Mesorektum, postuliert. Als vergleichbar radikales, jedoch kontinuitätserhaltendes Verfahren wurde die in der Folgezeit anteriore Rektumresektion von Dixon (1958) als Weiterentwicklung der von Reybeard (1843), Hochenegg (1900) und Weir (1901) beschriebenen Verfahren publiziert. Die 
Indikation zum Einsatz der beiden Verfahren erfolgt nach derzeitigem Kenntnisstand in Abhängigkeit von der Höhenlokalisation der Tumore und dem erzielbaren distalen Sicherheitsabstand. Hiernach wird heutzutage bei mehr als $1 \mathrm{~cm}$ distal tumorfreier Schleimhaut, gemessen am nativen nicht aufgespannten Präparat, kontinenzerhaltend, bei tiefer gelegenen Tumoren mittels Exstirpation operiert (Junginger 2004).

Beide radikalchirurgischen Ansätze entwickelten sich aufgrund niedriger Rezidivquoten von ca. 5 - 10\% (Heald et al. 1998, Heintz et al. 1997, Winde et al. 1996b, Mellgren et al. 2000, McDermott et al. 1985), zum Goldstandard der Therapie des Rektumkarzinoms. Den onkologischen Vorteilen der Radikalität steht allerdings das Problem einer hohen Morbidität und Mortalität gegenüber (Matzel et al. 2004, Sauer et al. 2004). So sind für die radikalchirurgischen Eingriffe Komplikationsraten zwischen $20 \%$ und $40 \%$ beschrieben (Stipa S et al. 2000, Winde et al. 1996a). Hinzu kommt die intra- und perioperative Letalität, die mit ca. 3\% - 4\% (Stipa et al. 2000, Salm et al. 1994), in Einzelfällen sogar mit bis zu 13\% (Kessler et al. 1993) angegeben wird. Weiterhin können postoperative Funktionsstörungen wie Harninkontinenz oder erektile Dysfunktionen bei bis zu 33\% (Kinn et Öhman 1986) sowie Stuhlinkontinenz bei bis zu 50\% (Carmona et al. 1991, Batignani et al. 1991) der Patienten auftreten. Erschwerend kommt hinzu, dass bis zu $75 \%$ der radikal operierten Patienten zumindest vorübergehend ein Stoma benötigen (Heald et al. 1998).

Aus den genannten Gründen wurden Verfahren zur lokalen Resektion der Tumore als Alternative zur radikalen Chirurgie entwickelt. Die bekanntesten hiervon sind die posterioren Zugänge nach Kraske (1887) und Mason (1976), sowie der transanale Zugang nach Parks (1970). Aufgrund der übermäßigen Traumatisierung zeigen die posterioren Zugänge jedoch Komplikationsraten von bis zu 30\% (Hermanek et Marzoli 1994), so dass beide Verfahren kaum noch Anwendung finden. Für den transanalen Zugang sind im Gegensatz hierzu zwar niedrige Komplikationsraten von etwa $5 \%$ beschrieben (Mörschel et al. 1998, Winde et al. 1996b), die Rezidivquote liegt mit bis zu 25\% (Sakamoto et al. 1991, Taylor et al. 1998) jedoch deutlich über jener der radikalen Chirurgie. Einschränkend kommt hinzu, dass der konventionellen transanalen Resektion nur Tumore des unteren Rektums zugänglich sind. 
Im Jahr 1983 stellte Buess als weitere Alternative für die lokale Resektion die so genannte transanale endoskopische Mikrochirurgie (TEM) vor (Buess et al. 1983, Buess et al. 1988a). Mit dieser Methode können Tumore des gesamten Rektums bei Komplikationsraten von ca. 5\% (Buess et al. 1988b) und einer von der Tumorgröße und -entität abhängigen Rezidivquote zwischen 4\% und 14\% (Langer et al. 2001b, Mentges et al. 1997, Saclarides 1997) operiert werden. Der Tumor wird hierbei durch ein starres Rektoskop unter Insufflation mit Gas zur Aufweitung des Rektums mittels mikrochirurgischer Instrumente, üblicherweise mit einer elektrischen Nadel, in Form einer Vollwandexzision exzidiert und der entstandene Defekt durch fortlaufende Naht verschlossen. Vorteile des so erzeugten Pneumorektums sind die herausragende Übersichtlichkeit und die Möglichkeit Tumore im gesamten Rektum zu operieren.

Alternativ zur elektrischen Nadel kann jedoch auch die Ultraschalldissektionstechnik in Form des so genannten UltraCision ${ }^{\circledR}$-Gerätes eingesetzt werden, bei dem die Energie zur Gewebsdissektion nicht thermisch, sondern kinetisch übertragen wird (Amaral 1994). Möglich ist diese Übertragung durch eine konstante Schwingung der Instrumentenklinge von $55 \mathrm{kHz}$. Durch diese Technik kann die Übersichtlichkeit im Operationsgebiet sowohl Einzelfallbeschreibungen (Kanaya et al. 1998) als auch retrospektiven Studien (Langer et al. 2001a) zufolge durch geringere Rauchentwicklung und weniger Blutungen weiter optimiert werden.

Ob und inwieweit lokale Verfahren neben der Behandlung benigner Tumore auch zur Therapie von Karzinomen des Rektums indiziert sind, ist Gegenstand aktueller Diskussionen. Eine differenzierte Therapie sollte unter Einhaltung der nötigen Radikalität den höchstmöglichen Patientenkomfort bieten. Das onkologisch definierte Maß chirurgischer Radikalität wird im Wesentlichen durch die Wahrscheinlichkeit einer Tumorinfiltration der Lymphknoten bestimmt. Eine solche Infiltration ist nur in etwa 5\% aller pT1-Karzinome (Hermanek et Gall 1986) zu erwarten. Innerhalb der pT1-Karzinome steigt diese Wahrscheinlichkeit jedoch sprunghaft von ca. $5 \%$ bei guter bis mäßiger Differenzierung (G1 - G2) auf bis zu 30\% bei schlecht differenzierten (G 3) oder entdifferenzierten (G4) Karzinomen an (Blumberg et al. 1999, Brodsky et al. 1992, Kraemer et al. 2001). Aus diesem Grund werden sowohl pT1 als auch höhergradige Karzinome dem Differenzierungsgrad nach in sogenannte low- und high-risk-Karzinome unterschieden (Hermanek et al. 1999). Neben der Infiltrationstiefe gilt auch die Infiltration von Lymphgefäßen (L1) als Risikofaktor für 
eine lokoregionäre Metastasierung. Da das Metastaserisiko bereits bei pT2-low-riskKarzinomen auf ca. $20 \%$ ansteigt (Nascimbeni et al. 2002), besteht nach Maßgabe der Deutschen Krebsgesellschaft (Junginger 2004) eine Indikation zur lokalen Therapie von Rektumtumoren aktuell nur für benigne Tumore, Carcinomata in situ und pT1-low-risk-Karzinome ohne Verdacht auf Lymphgefäßinvasion (LO) oder lokoregionäre Lymphknotenmetastasierung (NO).

Das Ziel dieser vergleichenden retrospektiven Studie ist es, die Frühergebnisse der TEM in der Therapie von Adenomen, Carcinomata in situ und pT1-low-riskKarzinomen des Rektums zu untersuchen. Hierzu soll ein Vergleich der TEM mit der radikalen Chirurgie als Goldstandard einerseits und der konventionellen Abtragung nach Parks als dem bis dato gebräuchlisten lokalen Verfahren andererseits vorgenommen werden. Für das TEM-Kollektiv soll darüber hinaus übergeprüft werden, ob der Einsatz der Ultraschalldissektionstechnologie als Alternative zur Anwendung der Hochfrequenzstromdissektion zu einer Verbesserung der Ergebnisse beitragen kann. 


\section{Material und Methoden}

\subsection{Zusammensetzung des Patientenkollektivs}

In das untersuchte Patientenkollektiv wurden alle Patienten aufgenommen, die mit der Diagnose eines

- benignen Tumors

- Carcinoma in situ (pTis)

- pT1-low-risk-Karzinoms

des Rektums im Zeitraum zwischen 1.1.1990 und 31.12.2001 in der Klinik und Poliklinik für Allgemeinchirurgie operiert wurden.

\section{Stratifizierung der Zielgruppe}

Die in der Zielgruppe enthaltenen Operationen wurden ihrer Art nach in 3 Hauptgruppen gegliedert:

- RC Radikale Chirurgie (anteriore Rektumresektion, Rektumexstirpation)

- TP Konventionelle transanale Resektion nach Parks

- TEM Transanale endoskopische Mikrochirurgie.

Die Hauptgruppe TEM wurde abhängig von der Art der verwendeten Technik weiter in Untergruppen differenziert:

- TEM-HF TEM mit Hochfrequenzstrom

- TEM-UC TEM mit Ultraschalltechnik (UltraCision $\left.{ }^{\circledR}\right)$.

\subsection{Präoperative Maßnahmen}

\section{Rektale Untersuchung}

Alle Patienten wurden präoperativ einer vollständigen klinischen Untersuchung inklusive rektal-digitaler Untersuchung zugeführt. Zur Bestimmung der Tumorlokalisation und Gewinnung einer präoperativen Biopsie wurde eine Rektoskopie mit starrem Rektoskop durchgeführt. Alle Tumore, deren Unterrand hierbei bis $16 \mathrm{~cm}$ von der Anokutanlinie gemessen wurde, galten als Rektumtumore. Als Kriterium für lokale Resektabilität galt die histopathologische Diagnose eines benignen Tumors, Carcinoma in situ oder gut bis mäßig differenzierten pT1-Karzinoms (G1-2). 


\section{Endosonografische Untersuchung}

Jeder Tumor wurde weiterhin endosonografisch auf den Grad der Tumorinvasion in die Rektumwand (ueT), sowie die Nachweisbarkeit malignitätsverdächtiger lokoregionärer Lymphknoten (ueN) hin untersucht. Hierfür wurde bis August 1999 ein 6004 Scanner (Brüel \& Kjaer, Norderstedt, Deutschland) mit einem 7-MHz Transducer und rotierender $90^{\circ}$ Sonde verwendet. Seit September 1999 wurde ein modernerer 3535 Scanner (Brüel \& Kjaer, Norderstedt, Deutschland) mit 10-MHz Transducer eingesetzt. Als lokal resektabel galten solche Tumore, bei denen kein Durchbruch der Submukosa, das heißt keine Infiltration der Tunica muscularis propria erbracht werden konnte (ueT 0 oder ueT1). Zusätzlich durfte bezüglich der lokoregionären Lymphknoten kein Anhalt für Malignität (ueN -) bestehen. Als Kriterien hierfür galten eine Größe von mehr als $1 \mathrm{~cm}$, ein scharf begrenzter Rand sowie ein homogen echoarmes Reflexmuster (Beynon et al. 1986, Hildebrandt et Feifel 1985, Hildebrandt et al. 1994).

Karzinome mit einer Infiltrationstiefe größer als ueT 1 oder Verdacht auf Lymphknoteninfiltration $(\mathrm{ueN}+$ ) wurden den nicht lokal resektablen Tumoren zugeordnet.

Die Tabelle 1 gibt einen Überblick über Tumore, die nach histopathologischem oder endosonografischem Befund als lokal resektabel beurteilt wurden.

Tabelle 1: Kriterien lokaler Resektabilität

\begin{tabular}{lllll}
\hline Biopsie & G & ueT & ueN & lokal resektabel \\
\hline benigne & & $\leq 1$ & - & Ja \\
pTis & & $\leq 1$ & - & Ja \\
Karzinom & $1-2$ & $\leq 1$ & - & Ja \\
Karzinom & $1-2$ & $\leq 1$ & + & Nein \\
Karzinom & $3-4$ & $\leq 1$ & - & Nein \\
Karzinom & $1-4$ & $>1$ & - & Nein \\
\hline
\end{tabular}

\section{Operationsvorbereitung}

Zum Ausschluss von Zweittumoren des Kolons wurde grundsätzlich eine Koloskopie oder eine Röntgenkonstrastuntersuchung mit Gastrografin durchgeführt. Präoperativ erfolgte bei allen Patienten, soweit keine Kontraindikationen bestanden, eine orale Darmspülung mit Polyethylenglycol (Oralav ${ }^{\circledR}$, Braun, Melsungen, Deutschland). Alternativ erfolgte insbesondere bei älteren und multimorbiden Patienten lediglich ein rektaler Natriumphosphateinlauf. Prophylaktisch wurde 30 min vor Operationsbeginn 
eine Singleshotantibiose mit $2 \mathrm{~g}$ Ceftriaxon (Ratiopharm, Ulm, Deutschland) sowie $0,5 \mathrm{~g}$ Metronidazol (Clont ${ }^{\circledR}$, Bayer vital $\mathrm{GmbH}$, Leverkusen, Deutschland) verabreicht.

\subsection{Operationsverfahren}

\section{Transanale Abtragung nach Parks (TP)}

Für die Durchführung der konventionellen transanalen Abtragung nach Parks wurden die Patienten in Steinschnittlage auf dem Operationstisch gelagert. Nach Vordehnung des Sphinkters und Einführen des Parkspreizers wurde die Schnittgrenze mit $1 \mathrm{~cm}$ Sicherheitsabstand um den Tumor mit der elektrischen Nadel markiert. Die Resektion des Tumors erfolgte grundsätzlich als Vollwandexzision. Bei Tumoren, die nicht in toto abgetragen werden konnten, wurde eine Abtragung in mehreren Teilstücken nach der so genannten piecemeal-Technik vorgenommen.

\section{Transanale endoskopische Mikrochirurgie (TEM)}

Für die Durchführung der TEM wurden die Patienten, der Lokalisation des Tumors entsprechend, in Seiten-, Bauch- oder Steinschnittlage so auf dem Operationstisch gelagert, dass der Tumor grundsätzlich in $6^{\circ}$ Position zu liegen kam. Nach Vordehnung des Sphinkters wurde das von Buess (Buess et al. 1983) beschriebene Instrumentarium mit starrem TEM-Rohr eingeführt und das Rektum mit $\mathrm{CO}_{2}$ insuffliert.

Die Markierung der Resektionsgrenzen mit $1 \mathrm{~cm}$ Sicherheitsabstand um den Tumor wurde grundsätzlich mit der elektrischen Nadel vorgenommen. Bis Mai 1996 wurde auch die Resektion des Tumors mit diesem Instrument (TEM-HF) durchgeführt. Seit Juni 1996 erfolgte die Resektion mit Hilfe eines Ultraschallskalpells (UltraCision ${ }^{\circledR}$, Ethicon, Sommerville, USA; TEM-UC). Bei beiden Techniken erfolgte grundsätzlich eine Vollwandexzision.

\section{Radikale Chirurgie (RC)}

Die radikalen Operationen wurden je nach Höhenlokalisation des Tumors als anteriore Rektumresektion oder Rektumexstirpation nach den Richtlinien der Deutschen Krebsgesellschaft (DKG) durchgeführt (Junginger 2004). Intraoperativ wurden nach Medianlaparatomie und Mobilisation des linken Hemikolons die A. und 
V. mesenterica inferior nach individueller Entscheidung des Operateurs gegebenenfalls unter Erhalt der A. colica sinistra ligiert. Bei Tumoren im unteren und mittleren Rektumdrittel erfolgte grundsätzlich eine totale mesorektale Exzision, im oberen Drittel eine partielle Exzision des Mesorektums bis $5 \mathrm{~cm}$ unterhalb des Tumors. Hierbei wurde ein aboraler Sicherheitsabstand von mindestens $1 \mathrm{~cm}$ zum Tumor, gemessen am nativen nicht aufgespannten Präparat, angestrebt. In Fällen, in denen ein solcher Sicherheitsabstand nicht erreichbar schien, oder intraoperativ nicht eingehalten werden konnte, wurde eine abdominoperineale Exstirpation vorgenommen. Die Deszendorektostomie erfolgte als End- zu Endanastomose in sogenannter Doublestapling-Technik mit einem handelsüblichen Klammernahtgerät (Ethicon, Norderstedt, Deutschland). Über die Notwendigkeit der Anlage protektiver Stomata wurde nach Einschätzung des Operateurs individuell entschieden.

\subsection{Histopathologische Begutachtung}

\section{Aufarbeitung der Tumore}

Alle Resektate wurden postoperativ in Formalin fixiert. Seit Juni 2000 wurden lokal resezierte Tumore vor der Aufarbeitung in frischem Zustand mit Nadeln auf Korkplatten befestigt. Die Fixierung erfolgte für 12 Stunden in Formalin, das im anschließenden Arbeitsgang über eine aufsteigende alkoholische Reihe (75\% Ethanol, 96\% Ethanol, 100\% Ethanol, Xylol) durch Paraffin ersetzt wurde. Aus dem eingesandten Gewebe wurden $5 \mu \mathrm{m}$ dicke repräsentative Schnitte angefertigt, die durch absteigende alkoholische Reihe (Xylol, 100\% Ethanol, 96\% Ethanol, 75\% Ethanol, Aqua dest.) entparaffiniert und anschließend mit Hämatoxylin/Eosin (Romeis 1989) gefärbt wurden.

\section{Klassifikation der Tumore}

Alle Tumore wurden entsprechend der TNM-Klassifikation (Hermanek et al. 1999) eingeteilt. Zusätzlich wurde für alle Tumore der Grad der Differenzierung (Grading) bestimmt. Entsprechend den von Hermanek (Hermanek et Gall 1986) vorgestellten Kriterien zur Differenzierung von Adenokarzinomen wurde eine weitergehende Unterscheidung in sogenannte low-risk- oder high-risk-Karzinome vorgenommen. Alle pT1-Karzinome mit guter oder mäßiger Differenzierung (G1 oder G2), fehlender 
Lymphknoten- (NO) und Gefäßinfiltration (LO) wurden dem entsprechend den pT1low-risk-Karzinomen zugeordnet. Schlecht differenzierte oder anaplastische pT1Karzinome (G3 oder G4) und solche mit Lymphknoten- (N1 oder N2) oder Lymphgefäßbeteiligung (L1) wurden den pT1-high-risk-Karzinomen zugeordnet.

\subsection{Nachuntersuchung und Follow up}

Für die Nachsorge der Patienten wurde das von der DKG vorgeschlagene Schema herangezogen (Junginger 2004, Schmiegel et al. 2004), welches Tabelle 2 wiedergibt.

\section{Tabelle 2: Nachsorgeschema}

\begin{tabular}{llllllll}
\hline & \multicolumn{1}{c}{ Monate } \\
\multicolumn{1}{c}{ Untersuchung } & $\mathbf{6}$ & $\mathbf{1 2}$ & $\mathbf{1 8}$ & $\mathbf{2 4}$ & $\mathbf{3 6}$ & $\mathbf{4 8}$ & $\mathbf{6 0}$ \\
\hline Anamnese, Körperliche Untersuchung & $\mathrm{X}$ & $\mathrm{X}$ & $\mathrm{X}$ & $\mathrm{X}$ & $\mathrm{X}$ & $\mathrm{X}$ & $\mathrm{X}$ \\
Rektoskopie & $\mathrm{X}$ & $\mathrm{X}$ & $\mathrm{X}$ & & & & \\
Koloskopie & & \multicolumn{7}{c}{$\mathrm{X}$} & & & $\mathrm{X}$ \\
\hline
\end{tabular}

Die Daten der Nachuntersuchungen aller Patienten wurden nach Aktenlage dokumentiert.

Alle Patienten, für die entsprechende Nachuntersuchungen an der Universitätsklinik nicht vorlagen, wurden zeitgleich mit ihren behandelnden Hausärzten angeschrieben und zu einer entsprechenden Nachuntersuchung eingeladen. Konnte bis 4 Wochen nach Abgang der Nachricht kein Untersuchungsergebnis bei den behandelnden Hausärzten erfragt werden, wurden die Patienten telefonisch kontaktiert, befragt und erneut auf die versäumten Nachsorgetermine hingewiesen. Bei fehlenden oder den Kriterien nicht entsprechenden Nachuntersuchungen wurde das Intervall zwischen der Operation und der letzten vollständig ausgeführten Nachsorgeuntersuchung als Follow up verzeichnet.

\subsection{Datenverarbeitung}

Sämtliche Daten wurden in einer relationalen Datenbank (MS Access 2000 ${ }^{\circledR}$, Microsoft, Berlin, Deutschland) erfasst. 


\section{Struktur der Daten}

Bei den erfassten Informationen handelte es sich um Angaben über behandelnde Hausärzte, Patientenstammdaten, durchgeführte Operationen und den Nachsorgestatus. Dem Problem, dass ein Hausarzt mehrere Patienten betreute, von denen einige mehrfach operiert wurden, trug die eingesetzte Datenbank insofern Rechnung, als dass 3 hierarchisch gegliederte Haupttabellen existierten, in denen die Daten der Hausärzte, Patienten und Operationen getrennt voneinander erfasst wurden. In den 3 Haupttabellen hatte jeder Datensatz, beispielsweise ein Arzt oder eine Operation eine eindeutige, automatisch vergebene Nummer (Primärschlüssel), über die der Datensatz identifiziert werden konnte. Über eine zweite Eintragung (Fremdschlüssel) konnte einem Datensatz, z.B. einem Patienten, der Datensatz einer übergeordneten Tabelle, in diesem Falle eines Hausarztes, zugewiesen werden. Die Abbildung 1 zeigt schematisch die $1 \rightarrow \mathrm{n}$ Beziehungen der 3 Haupttabellen Arzt, Patient, Operation.

\section{Abbildung 1: Datenbankstruktur $1 \rightarrow \mathrm{n}$}

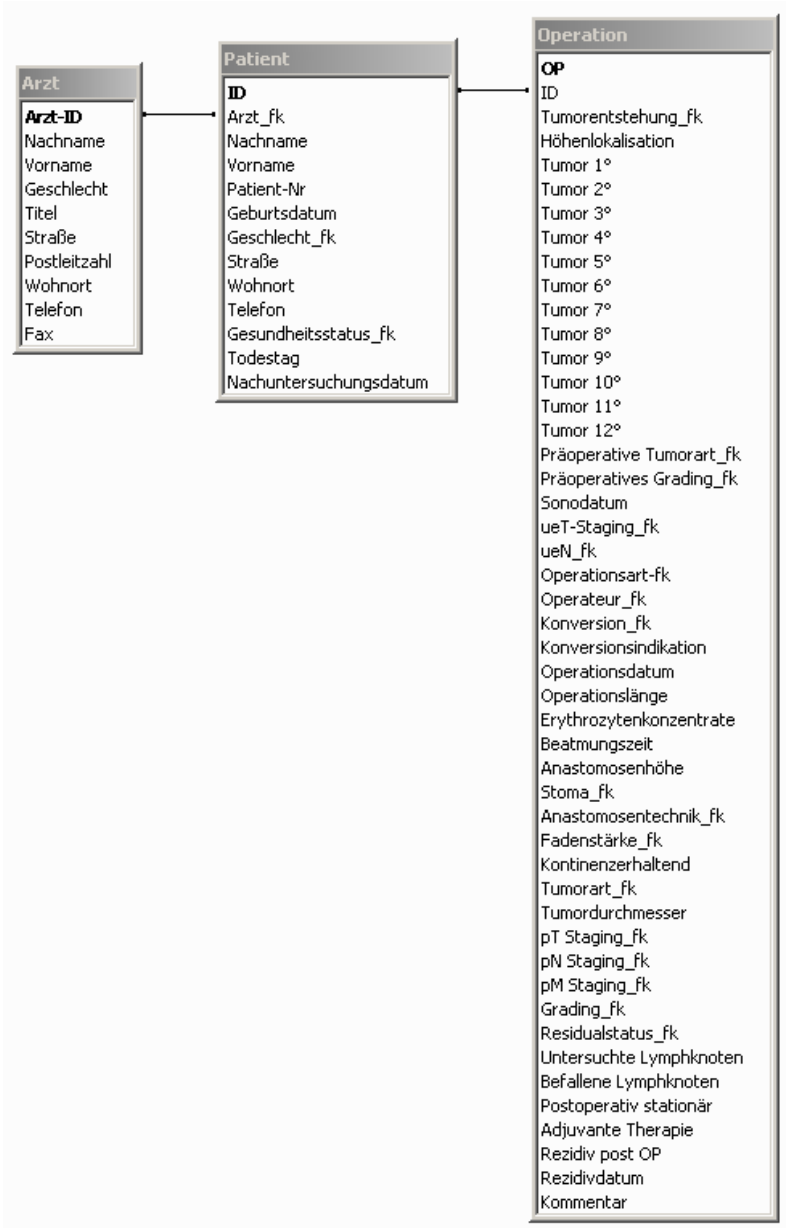


Für die Dokumentation einer Komplikation wurde eine andere logische Struktur verwendet, die es ermöglichte, jeder Operation verschiedene Komplikationen und zugleich jeder Komplikationsart mehrere Operationen zuzuordnen. Für jede Komplikation wurde der eindeutige, automatisch vergebene, Primärschlüssel in der Untertabelle Komplikation gespeichert. Die Operation, der dieses Ereignis zuzuordnen war, wurde als Fremdschlüssel gespeichert. Aus der Codetabelle Komplikationsart wurde die Art der Komplikation ebenfalls als Fremdschlüssel bezogen. Die Art der Behandlung jeder Komplikation wurde als Fremdschlüssel aus der Codetabelle Komplikationstherapie entnommen.

Die Abbildung 2 zeigt exemplarisch die sogenannte $\mathrm{n} \rightarrow \mathrm{m}$ Beziehung der Haupttabelle Operation mit den Codetabellen Komplikationsart und Komplikationstherapie und der als Verknüpfung dienenden Untertabelle Komplikation.

\section{Abbildung 2: Datenbankstruktur $n \rightarrow m$}

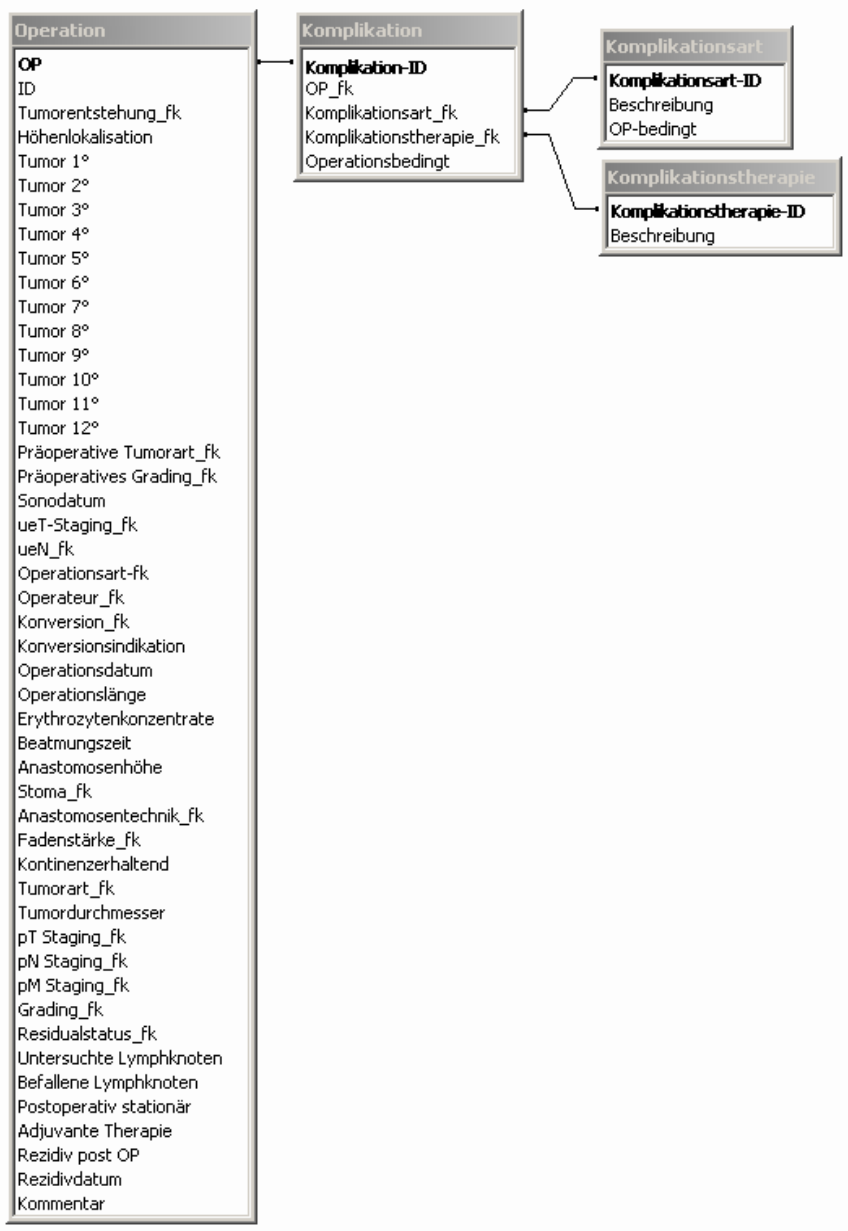




\section{Erfasste Daten}

Im Folgenden sind alle Daten, die in der verwendeten Datenbank bearbeitet wurden, zusammengefasst. Alle Tabellen sind als Tabellenentwürfe dargestellt. Hierbei gibt jede Zeile der Tabellenentwürfe eine Spalte der tatsächlichen Tabellen wieder. Jede Zeile der tatsächlichen Tabellen entspricht hierbei einem Datensatz, zum Beispiel einem Arzt. Spaltennamen, die auf "_fk“ enden, sind Fremdschlüssel, die sich auf Codetabellen beziehen.

Tabelle 3: Daten der Ärzte

\begin{tabular}{l}
\hline Spaltenname \\
\hline Arzt-ID \\
Nachname \\
Vorname \\
Geschlecht_fk \\
Titel_fk \\
Straße \\
Postleitzahl \\
Wohnort \\
Telefon \\
Fax \\
\hline
\end{tabular}

Tabelle 5: Daten der Patienten

\begin{tabular}{l}
\hline Spaltenname \\
\hline Patient-ID \\
Arzt_fk \\
Nachname \\
Vorname \\
Patientnummer \\
Geburtsdatum \\
Geschlecht_fk \\
Titel_fk \\
Straße \\
Postleitzahl \\
Wohnort \\
Telefon \\
Gesundheitsstatus_fk \\
Nachuntersuchungsdatum \\
Todestag \\
\hline
\end{tabular}

Tabelle 6: Daten der Komplikationen

\begin{tabular}{l}
\hline Spaltenname \\
\hline Komplikation-ID \\
OP_fk \\
Komplikationsart_fk \\
Behandlung_fk \\
Operationsbedingt \\
\hline
\end{tabular}

Tabelle 4: Daten der Operationen

\begin{tabular}{|c|}
\hline Spaltenname \\
\hline Operations-ID \\
\hline Patient_fk \\
\hline Tumorentstehung_fk \\
\hline Höhe \\
\hline Tumor $1^{\circ}$ \\
\hline Tumor $2^{\circ}$ \\
\hline Tumor $3^{\circ}$ \\
\hline Tumor $4^{\circ}$ \\
\hline Tumor $5^{\circ}$ \\
\hline Tumor $6^{\circ}$ \\
\hline Tumor $7^{\circ}$ \\
\hline Tumor $8^{\circ}$ \\
\hline Tumor $9^{\circ}$ \\
\hline Tumor $10^{\circ}$ \\
\hline Tumor $11^{\circ}$ \\
\hline Tumor $12^{\circ}$ \\
\hline Biopsie Tumorart_fk \\
\hline Biopsie Grading_fk \\
\hline Sonodatum \\
\hline ueT_fk \\
\hline ueN_fk \\
\hline Operationsart_fk \\
\hline Operationsdatum \\
\hline Operationslänge \\
\hline Operateur_fk \\
\hline Konversion_fk \\
\hline Konversionsindikation \\
\hline Anastomosenhöhe \\
\hline Stoma_fk \\
\hline Kontinēnzerhaltend \\
\hline Anastomosentechnik_fk \\
\hline Fadenstärke_fk \\
\hline Erythrozytenkonzentrate \\
\hline Beatmungszeit \\
\hline Postoperativ stationär \\
\hline Tumorart_fk \\
\hline Größe - \\
\hline pT-Staging_fk \\
\hline pN-Staging_fk \\
\hline Untersuchte Lymphknoten \\
\hline Befallene Lymphknoten \\
\hline pM-Staging_fk \\
\hline pM Lokalisation \\
\hline Grading_fk \\
\hline Residualstatus_fk \\
\hline Rezidiv \\
\hline Rezidivdatum \\
\hline Kommentar \\
\hline
\end{tabular}




\section{Statistische Auswertung und grafische Darstellung der Daten}

Die Auswertung erfolgte in Zusammenarbeit mit der Abteilung für medizinische Statistik unter Verwendung des Programms SAS ${ }^{\circledR}$ (Version 8.0, SAS Institute $\mathrm{GmbH}$, Heidelberg, Deutschland). Für die statistische Analyse wurden zunächst Paarvergleiche der Hauptgruppen RC und TP versus TEM und der Untergruppen TEM-HF versus TEM-UC durchgeführt. Alle Nullhypothesen wurden zweiseitig zum lokalen Niveau von $5 \%$ getestet. Bei dichotomen Zielvariablen wurde der exakte Fisher Test verwendet. Lag die Zielvariable auf einer nominalen Skala mit mehr als 2 Kategorien, wurde der $\chi^{2}$-Test durchgeführt. Bei intervall- oder rationalskalierten Zielvariablen wurde der Wilcoxon-Test angewandt. Mehrfaktorielle Analysen für dichotome Zielvariablen wurden als logistische Regression durchgeführt. Bei ordinalen Zielvariablen wurde zusätzlich das propotional-odds-model verwendet. In die Modelle der multifaktoriellen Analysen wurden nur die Faktoren aufgenommen, die schon im einfaktoriellen Design einen Einfluss zeigten.

Die grafische Darstellung der Daten wurde mit MS Excel $2000^{\circledR}$ (Microsoft, Berlin, Deutschland) vorgenommen.

Die Ergebnisse der deskriptiven Statistik nominaler Variablen werden im Text der Übersichtlichkeit halber auf eine Stelle nach dem Komma gerundet angegeben. Abweichungen von $100 \%$ in der Summierung der Einzelgruppen resultieren aus dieser Rundung. Für die Ergebnisse der deskriptiven Statistik rationaler Variablen wird die folgende Schreibweise verwendet: (Mittelwert \pm Standardabweichung [Minimum - Maximum]) 


\section{$3 \quad$ Ergebnisse}

\subsection{Zusammensetzung des Kollektivs}

\section{Anzahl der Zielgruppenpatienten und Operationen}

Es wurden Daten über 161 Patienten erhoben, an denen insgesamt 182 Operationen vorgenommen wurden.

\section{Anzahl der mehrfach operierten Patienten}

Von den 182 Operationen entfielen 145 auf jeweils einen einzelnen Patienten. An 13 Patienten wurden 2, an 1 Patienten 3 und an 2 Patienten 4 Operationen durchgeführt.

\section{Gruppenbildung}

Die 182 Operationen verteilten sich auf die untersuchten Hauptgruppen mit 27 $(14,8 \%)$ auf RC, $76(41,8 \%)$ auf TP und 79 (43,3\%) auf TEM. Innerhalb der Hauptgruppe TEM waren 45 (57,0\%) Operationen der Untergruppe TEM-HF und 34 $(43,0 \%)$ der Untergruppe TEM-UC zugehörig.

\subsection{Vergleichskriterien}

Im Folgenden werden die Kriterien zur Untersuchung der statistischen Vergleichbarkeit der Haupt- und Untergruppen nach

\subsubsection{Alter}

3.2.2 Geschlecht

3.2.3 Tumorentstehung

3.2.4 Histologie

3.2.5 Größe der Tumore

3.2.6 Höhenlokalisation des Tumors ab ano

3.2.7 Lokalisation des Tumors bezogen auf die Zirkumferenz des Rektums

3.2.8 Residualstatus

3.2.9 Nachsorgezeitraum

beschrieben. 


\subsubsection{Alter}

Das Alter der Patienten zum Zeitpunkt der Operation betrug in den Hauptgruppen für RC 67,3 \pm 12,7 [36,0 - 88,2], für TP 64,7 $\pm 17,1$ [13,0 - 90,7] und für TEM 64,7 $\pm 10,9$ [25,6 - 84,1] Jahre. In den Untergruppen wurden Werte von 66,6 $\pm 10,5[25,6-84,1]$ für TEM-HF und 62,1 $\pm 11,2$ [28,8 - 78,2] für TEM-UC bestimmt.

Im Wilcoxon-Test konnte weder für den Vergleich der Hauptgruppen noch für den Vergleich der Untergruppen ein signifikanter Altersunterschied nachgewiesen werden.

Die Abbildung 3 zeigt die Anzahl der nach dem Alter bei Operation in Zehnjahresintervallen geschichteten Patienten jeder Operationsart.

\section{Abbildung 3: Alter}

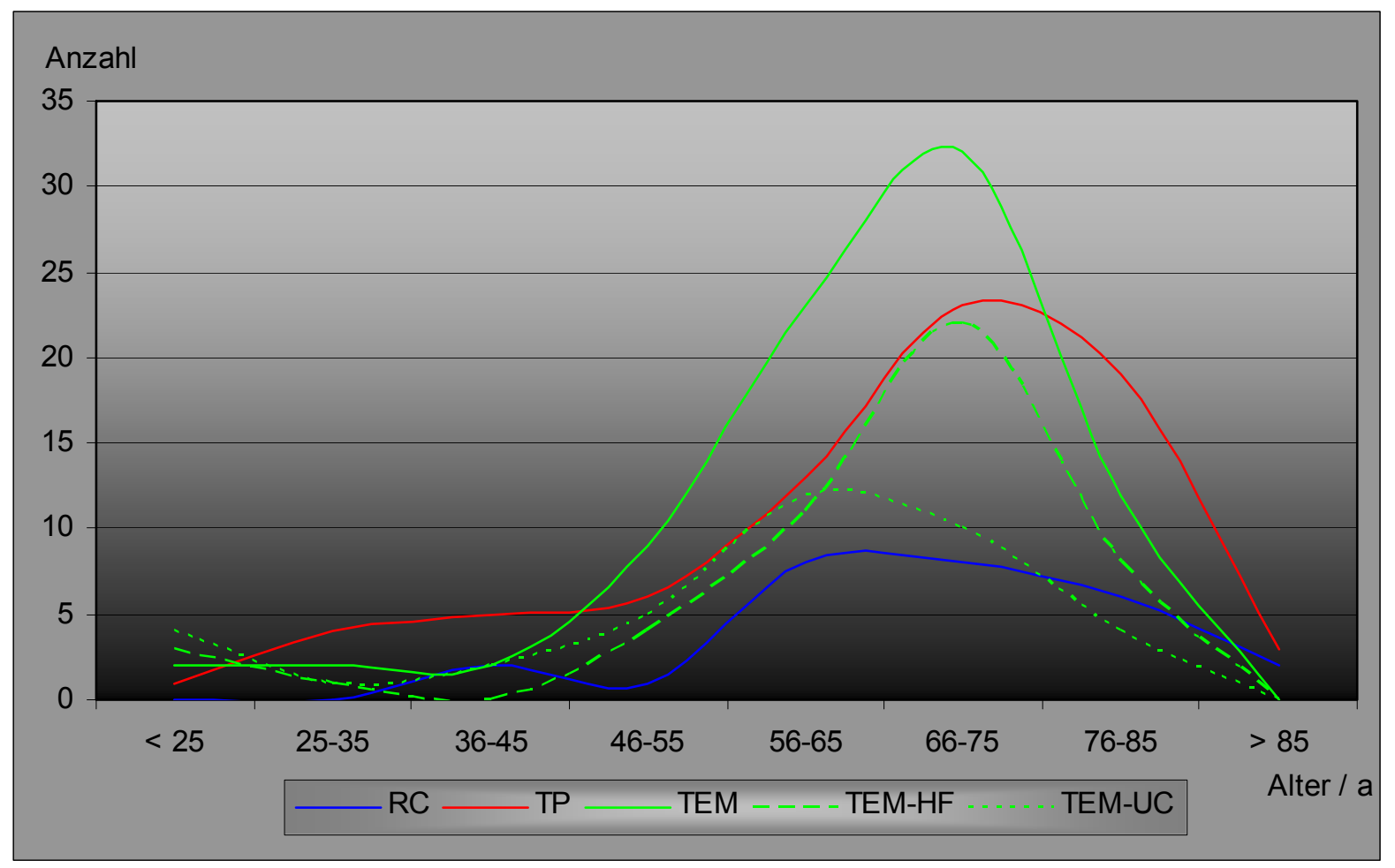

\subsubsection{Geschlecht}

Das Geschlecht der Patienten wurde nach Haupt- und Untergruppen getrennt auf ihre Anzahl und prozentuale Verteilung hin untersucht. Die Ergebnisse dieser Untersuchung sind in Tabelle 7 für jedes der Operationsverfahren einzeln sowie insgesamt zusammengefasst. 
Tabelle 7: Geschlecht

\begin{tabular}{ccc}
\hline \multicolumn{3}{c}{ Hauptgruppen } \\
\hline & Männlich & Weiblich \\
\hline RC & 15 & 12 \\
& $55,6 \%$ & $44,4 \%$ \\
TP & 27 & 49 \\
& $35,5 \%$ & $64,5 \%$ \\
TEM & 44 & 35 \\
& $55,7 \%$ & $44,3 \%$ \\
Gesamt & 86 & 96 \\
& $47,3 \%$ & $52,7 \%$ \\
\hline
\end{tabular}

\begin{tabular}{lcc}
\hline & Untergruppen \\
\hline & Männlich & Weiblich \\
\hline \multirow{2}{*}{ TEM-HF } & 28 & 17 \\
& $62,2 \%$ & $37,8 \%$ \\
TEM-UC & 16 & 18 \\
& $47,1 \%$ & $52,9 \%$ \\
Gesamt & 44 & 35 \\
& $55,7 \%$ & $44,3 \%$ \\
\hline
\end{tabular}

Der Vergleich der Hauptgruppe TEM mit RC und TP zeigte im exakten Fisher Test für TEM vs. TP mit einem $p$-Wert von 0,016 signifikant mehr Frauen innerhalb der Hauptgruppe TP. Für den Vergleich der Untergruppen TEM-HF vs. TEM-UC war kein statistisch signifikanter Unterschied nachweisbar.

\subsubsection{Tumorentstehung}

Für die Untersuchung der Tumorgenese wurde auf der Grundlage der ärztlichen Dokumentation nach Primärtumoren und Rezidiven unterschieden. Für jede Gruppe wurden hierauf basierend die Anzahl sowie der prozentuale Anteil bestimmt.

Die Verteilung auf Primärtumore und Rezidive kann der Tabelle 8 zusammenfassend entnommen werden.

Tabelle 8: Tumorentstehung

\begin{tabular}{|c|c|c|}
\hline \multicolumn{3}{|c|}{ Hauptgruppen } \\
\hline & Primär & Rezidiv \\
\hline RC & $\begin{array}{c}20 \\
74,1 \%\end{array}$ & $\begin{array}{c}7 \\
25,9 \%\end{array}$ \\
\hline TP & $\begin{array}{c}49 \\
46,5 \%\end{array}$ & $\begin{array}{c}27 \\
35,5 \%\end{array}$ \\
\hline TEM & $\begin{array}{c}67 \\
84,8 \%\end{array}$ & $\begin{array}{c}12 \\
15,2 \%\end{array}$ \\
\hline Gesamt & $\begin{array}{c}136 \\
74,7 \%\end{array}$ & $\begin{array}{c}46 \\
25,3 \%\end{array}$ \\
\hline
\end{tabular}

\begin{tabular}{lcc}
\hline & Untergruppen \\
\hline & Primär & Rezidiv \\
\hline \multirow{2}{*}{ TEM-HF } & 36 & 9 \\
& $80,0 \%$ & $20,0 \%$ \\
TEM-UC & 31 & 3 \\
& $91,2 \%$ & $8,8 \%$ \\
Gesamt & 67 & 12 \\
& $84,8 \%$ & $15,2 \%$ \\
\hline
\end{tabular}

Der Vergleich der Hauptgruppen mit dem exakten Fisher Test zeigte nur für TEM vs. TP einen signifikanten Unterschied ( $p$-Wert $=0,005)$ mit mehr Rezidiven in der Gruppe TP. Der Vergleich der Untergruppen TEM-HF und TEM-UC erbrachte keine Unterschiede. 
Die Abbildung 4 veranschaulicht den prozentualen Anteil der Primärtumore und Rezidive an der Gesamtheit aller Tumore eines Operationsverfahrens.

\section{Abbildung 4: Tumorentstehung}

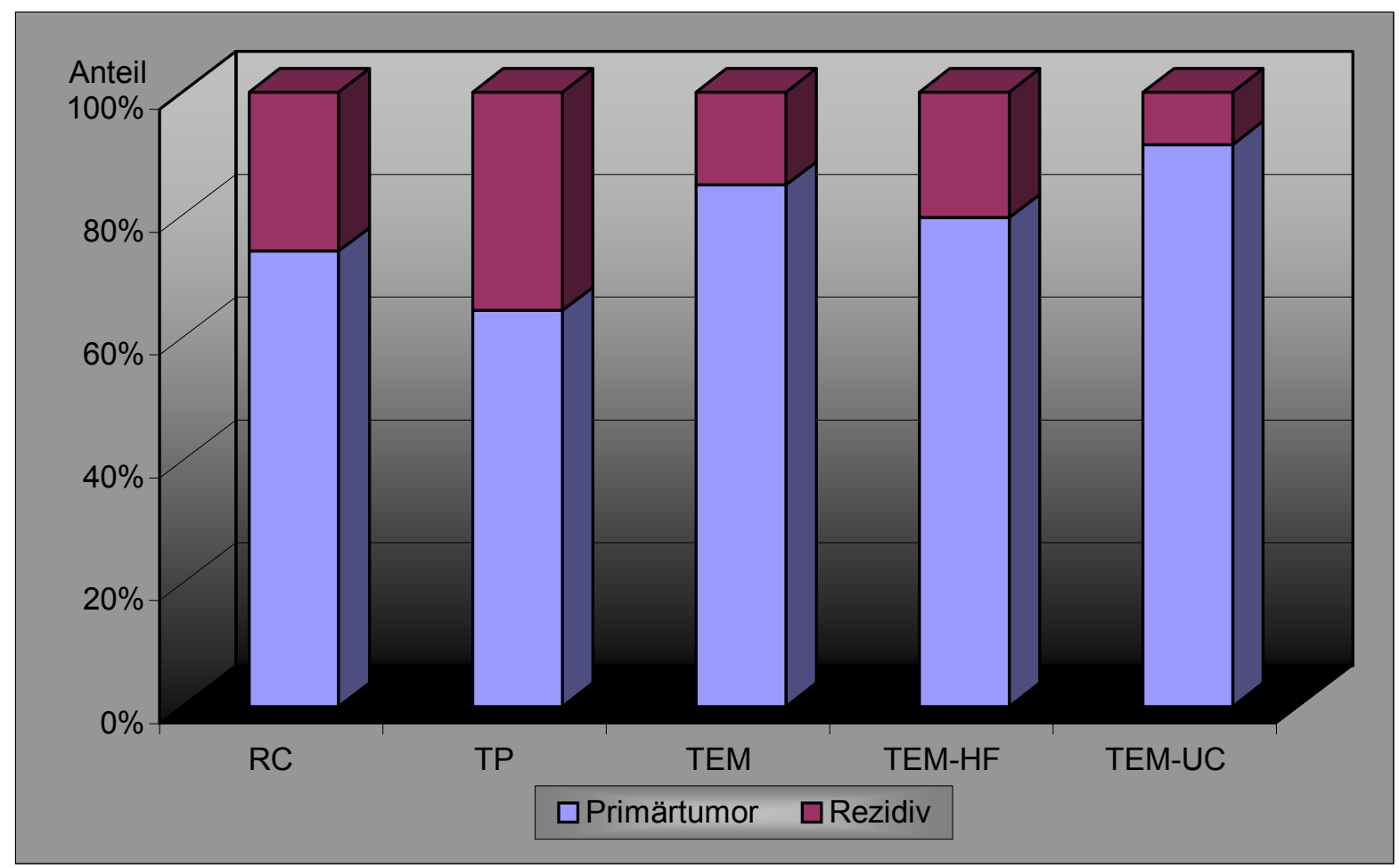

\subsubsection{Histologie}

Für den Vergleich der Histologie wurde den pathologischen Berichten die histologische Beschreibung der Tumorart, sowie das Staging gemäß der TNM Klassifikation entnommen. Die Anzahl sowie der prozentuale Anteil wurden auf der Basis dieser Daten bestimmt.

Die Tabelle 9 gibt die Verteilung der Tumorarten und des pathologischen Stagings auf die untersuchten Operationsverfahren zusammenfassend wieder.

Tabelle 9: Histologie

\begin{tabular}{|c|c|c|c|c|c|c|}
\hline \multicolumn{7}{|c|}{ Hauptgruppen } \\
\hline & \multicolumn{3}{|c|}{ Benigne } & \multicolumn{3}{|c|}{ Maligne } \\
\hline & Aden. & Sonst. & $\sum$ & pTis & $\mathrm{pT} 1$ & $\sum$ \\
\hline RC & $\begin{array}{c}8 \\
29,6 \%\end{array}$ & $\begin{array}{c}1 \\
3,7 \%\end{array}$ & $\begin{array}{c}9 \\
33,3 \%\end{array}$ & $\begin{array}{c}3 \\
11,1 \%\end{array}$ & $\begin{array}{c}15 \\
55,6 \%\end{array}$ & $\begin{array}{c}c \\
18 \\
66,7 \%\end{array}$ \\
\hline TP & $\begin{array}{c}53 \\
69,7 \%\end{array}$ & $\begin{array}{c}4 \\
5,3 \%\end{array}$ & $\begin{array}{c}57 \\
75,0 \%\end{array}$ & $\begin{array}{c}6 \\
7,9 \%\end{array}$ & $\begin{array}{c}13 \\
17,1 \%\end{array}$ & $\begin{array}{c}19 \\
25,0 \%\end{array}$ \\
\hline TEM & $\begin{array}{c}57 \\
72,2 \%\end{array}$ & $\begin{array}{c}2 \\
2,5 \%\end{array}$ & $\begin{array}{c}59 \\
74,7 \%\end{array}$ & $\begin{array}{c}8 \\
10,1 \%\end{array}$ & $\begin{array}{c}12 \\
15,2 \%\end{array}$ & $\begin{array}{c}20 \\
25,3 \%\end{array}$ \\
\hline Gesamt & $\begin{array}{c}118 \\
64,8 \%\end{array}$ & $\begin{array}{c}7 \\
3,8 \% \\
\end{array}$ & $\begin{array}{c}125 \\
68,7 \%\end{array}$ & $\begin{array}{c}17 \\
9,3 \%\end{array}$ & $\begin{array}{c}40 \\
22,0\end{array}$ & $\begin{array}{c}57 \\
31,3\end{array}$ \\
\hline
\end{tabular}




\begin{tabular}{ccccccc}
\hline \multicolumn{7}{c}{ Untergruppen } \\
\hline \multicolumn{7}{c}{ Benigne } \\
\cline { 2 - 7 } & Aden. & Sonst. & $\sum$ & pTis & pT1 & $\Sigma$ \\
\hline TEM- & 31 & 1 & 32 & 5 & 8 & 13 \\
HF & $68,9 \%$ & $2,2 \%$ & $71,1 \%$ & $11,1 \%$ & $17,8 \%$ & $28,9 \%$ \\
TEM- & 26 & 1 & 27 & 3 & 4 & 7 \\
UC & $76,5 \%$ & $2,9 \%$ & $79,4 \%$ & $8,8 \%$ & $11,8 \%$ & 21 \\
Gesamt & 57 & 2 & 59 & 8 & 12 & 20 \\
& $72,2 \%$ & $2,5 \%$ & $74,7 \%$ & $10,1 \%$ & $15,2 \%$ & $25,3 \%$ \\
\hline
\end{tabular}

Der Vergleich der Verteilung auf Adenome, Karzinome und sonstige Tumore im $\chi^{2}$ Test zeigte mit einem p-Wert von 0,001 einen signifikanten Unterschied für TEM vs. RC mit mehr Karzinomen in der Gruppe RC. Der Vergleich TEM vs. TP erbrachte ebenso wie der Vergleich der Untergruppen TEM-HF und TEM-UC keine signifikanten Unterschiede.

Die Abbildung 5 stellt den prozentualen Anteil der verschiedenen Tumorarten an der Gesamtheit aller Tumore jedes Operationsverfahrens grafisch dar.

\section{Abbildung 5: Histologie}

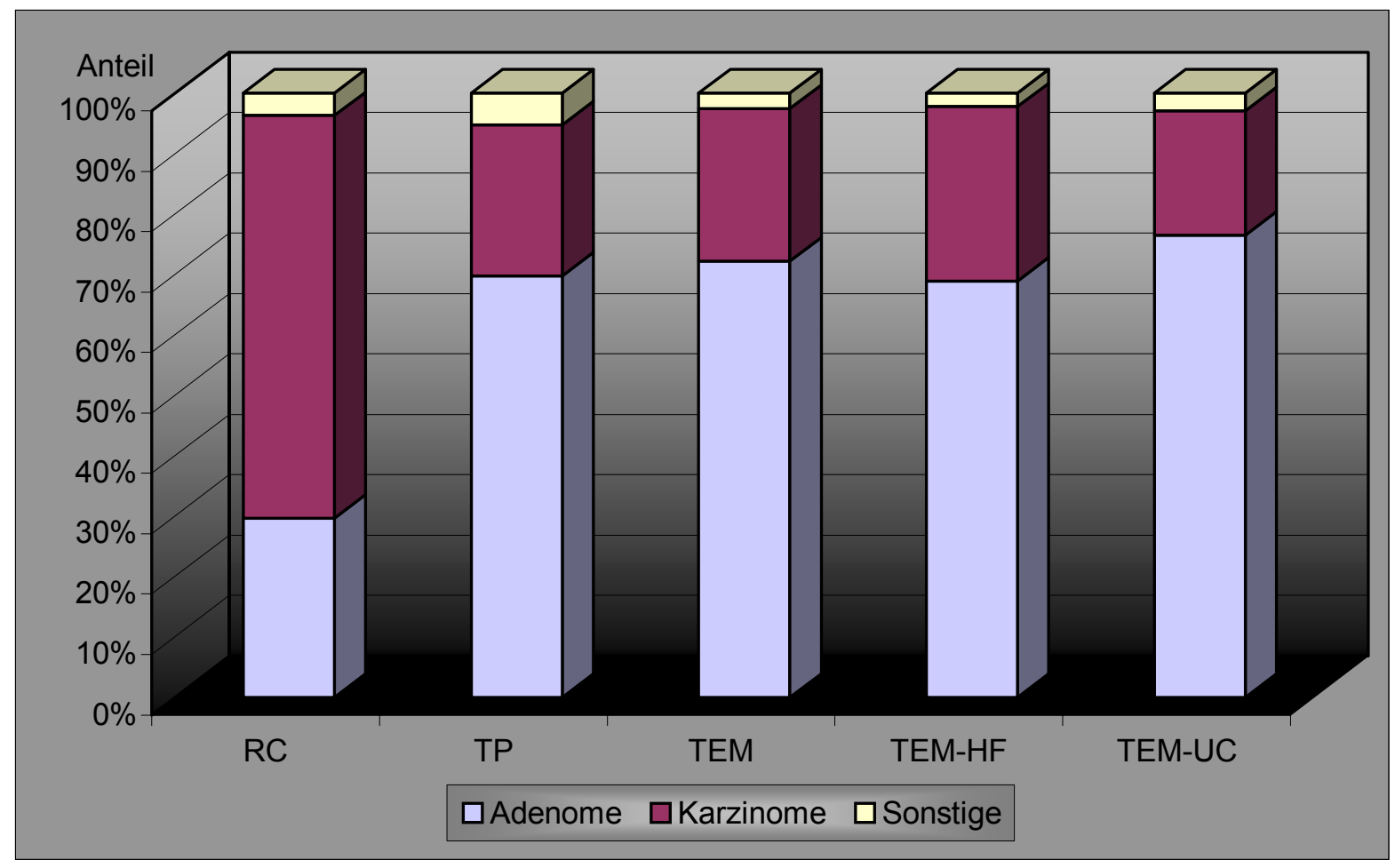


Für den Zeitraum seit Einführung der transanalen endoskopischen Mikrochirurgie wurde jeweils für Adenome und Karzinome ein Behandlungsprofil erstellt. Hierbei wurden die Jahre von 1995 bis 2001 jeweils einzeln betrachtet und die innerhalb des jeweiligen Jahres aufgetretenen Tumore nach der Art des verwendeten Operationsverfahrens auf der Zeitachse abgetragen.

Eine Übersicht über die Anzahl aller Adenome, die innerhalb des angegebenen Zeitraumes operiert wurden, kann synoptisch der Tabelle 10 und grafisch der Abbildung 6 entnommen werden.

Tabelle 10: Behandlung der Adenome von 1995 - 2001

\begin{tabular}{cccccccc}
\hline & $\mathbf{1 9 9 5}$ & $\mathbf{1 9 9 6}$ & $\mathbf{1 9 9 7}$ & $\mathbf{1 9 9 8}$ & $\mathbf{1 9 9 9}$ & $\mathbf{2 0 0 0}$ & $\mathbf{2 0 0 1}$ \\
\hline Gesamt & 9 & 17 & 16 & 11 & 21 & 17 & 6 \\
RC & 0 & 1 & 0 & 0 & 2 & 1 & 1 \\
TP & 5 & 13 & 10 & 4 & 1 & 4 & 1 \\
TEM & 4 & 3 & 6 & 7 & 18 & 12 & 4 \\
\hline
\end{tabular}

\section{Abbildung 6: Behandlung der Adenome von 1995 - 2001}

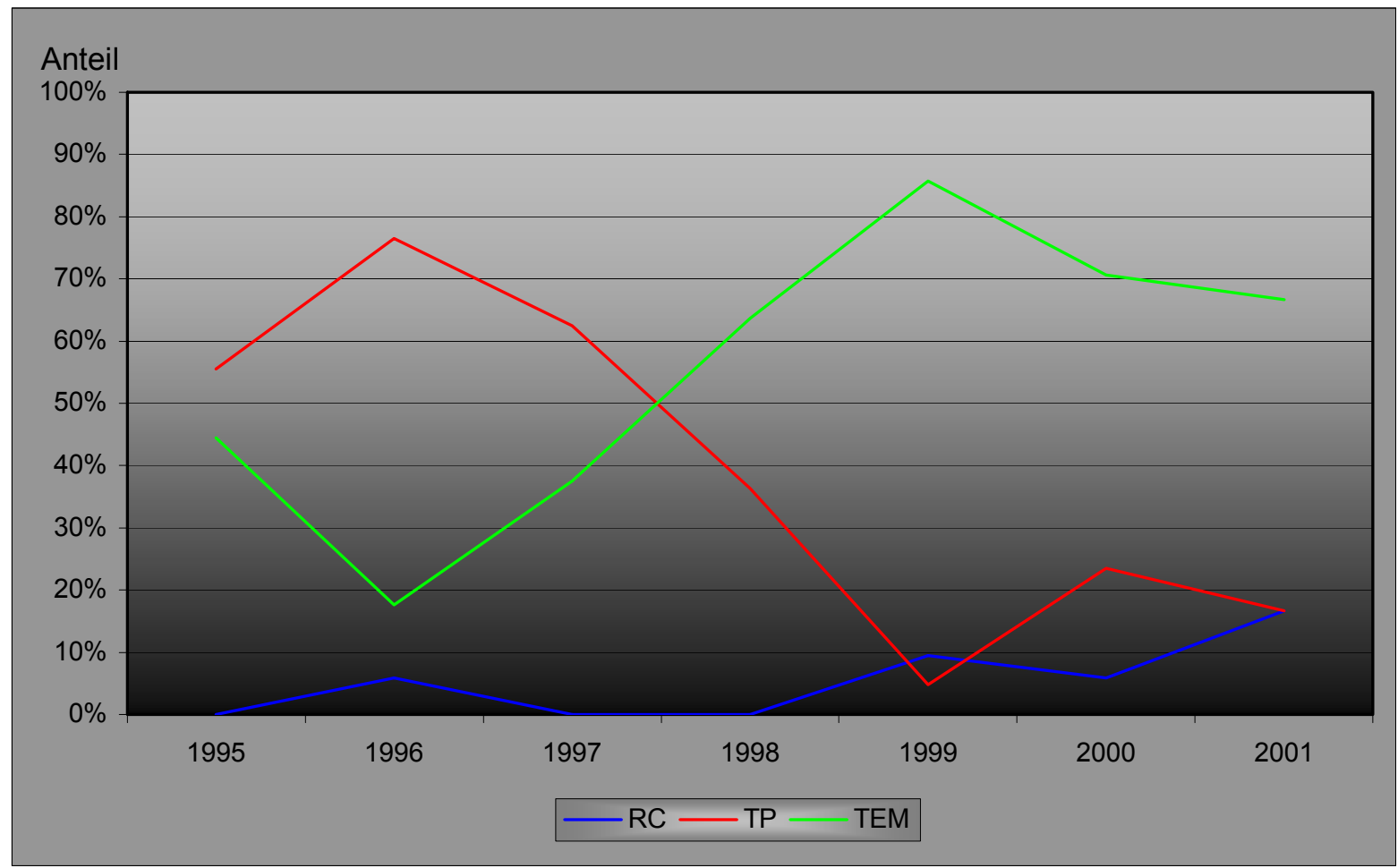

Die tabellarische und grafische Übersicht über den Einsatz der Operationsverfahren bei Karzinomen ist in der Tabelle 11 respektive in der Abbildung 7 wiedergegeben. 
Tabelle 11: Behandlung der Karzinome von 1995 - 2001

\begin{tabular}{cccccccc}
\hline & $\mathbf{1 9 9 5}$ & $\mathbf{1 9 9 6}$ & $\mathbf{1 9 9 7}$ & $\mathbf{1 9 9 8}$ & $\mathbf{1 9 9 9}$ & $\mathbf{2 0 0 0}$ & $\mathbf{2 0 0 1}$ \\
\hline Gesamt & 9 & 9 & 11 & 7 & 10 & 7 & 3 \\
RC & 6 & 3 & 5 & 1 & 4 & 0 & 0 \\
TP & 2 & 4 & 6 & 1 & 0 & 2 & 0 \\
TEM & 1 & 2 & 0 & 5 & 6 & 5 & 3 \\
\hline
\end{tabular}

\section{Abbildung 7: Behandlung der Karzinome von 1995 - 2001}

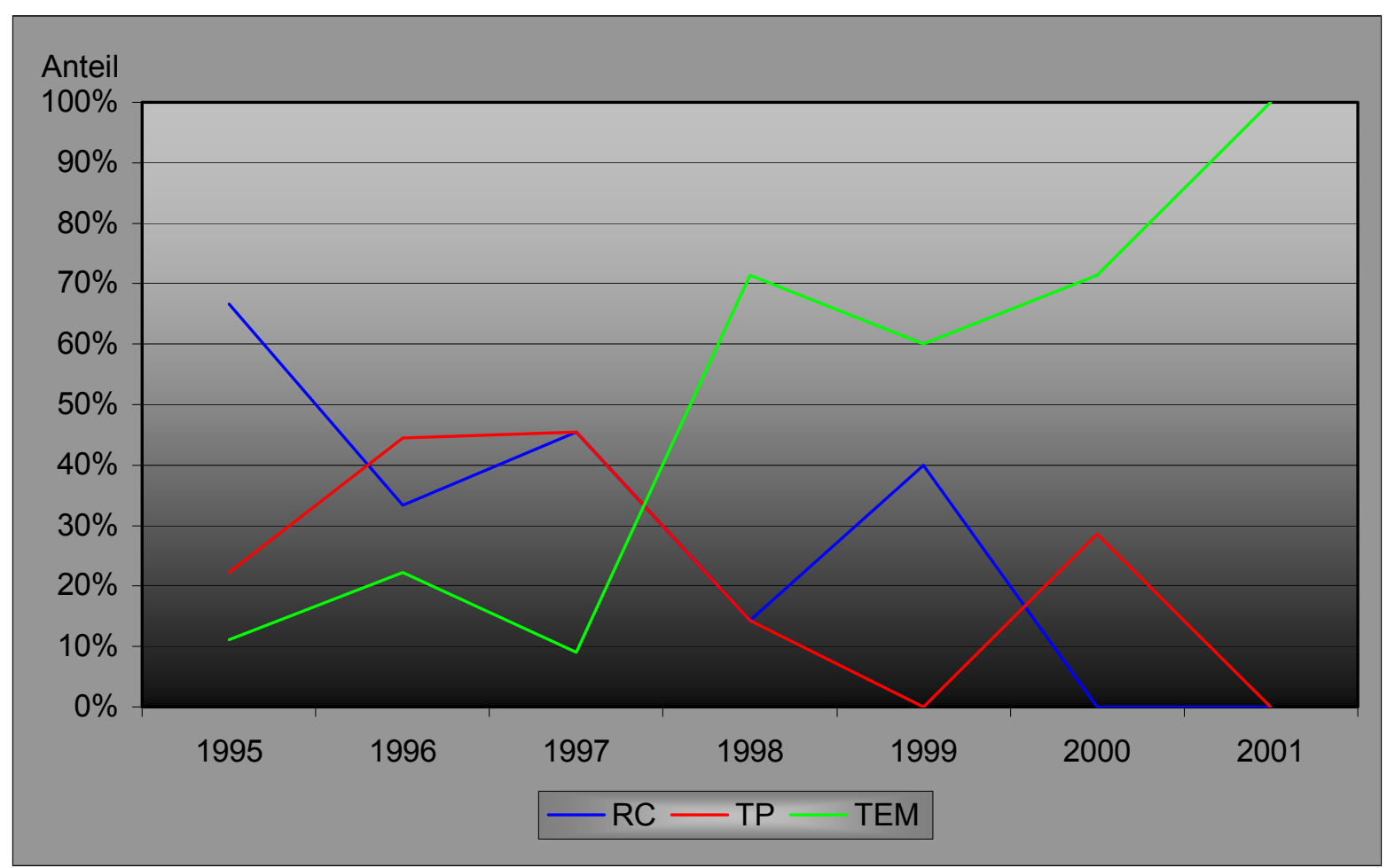

\subsubsection{Größe der Tumore}

Für die Untersuchung der Tumorgröße wurde den pathologischen Berichten der jeweils größte Durchmesser jedes resezierten Tumors entnommen. Die Tumore mit dem kleinsten Durchmesser wurden in der Gruppe TP mit 2,2 $\pm 1,5[0,1-8] \mathrm{cm}$ gefolgt von der Gruppe RC mit 3,2 cm $\pm 2,3[0,2-9] \mathrm{cm}$ verzeichnet. Die größten Tumore fanden sich in der Gruppe der TEM mit 3,4 $\pm 1,5[0,5-7] \mathrm{cm}$. Für die Untergruppe TEM-HF wurden Werte von 3,4 $\pm 1,5[0,5-7] \mathrm{cm}$ und für TEM-UC von $3,4 \pm 1,5[1-7] \mathrm{cm}$ verzeichnet.

Die Vergleichbarkeitsprüfung mit dem Wilcoxon-Test ergab für TEM vs. TP einen signifikanten Unterschied $(p$-Wert $=0,001)$ mit größeren Tumoren innerhalb der 
Gruppe TEM. Für die Vergleiche TEM vs. RC und TEM-HF vs. TEM-UC konnte kein signifikanter Unterschied nachgewiesen werden.

Die Abbildung 8 zeigt den prozentualen Anteil der nach Zentimeter Durchmesser kategorisierten Tumore für jede Gruppe, bezogen auf die Gesamtzahl aller untersuchter Tumore.

\section{Abbildung 8: Größe der Tumore}

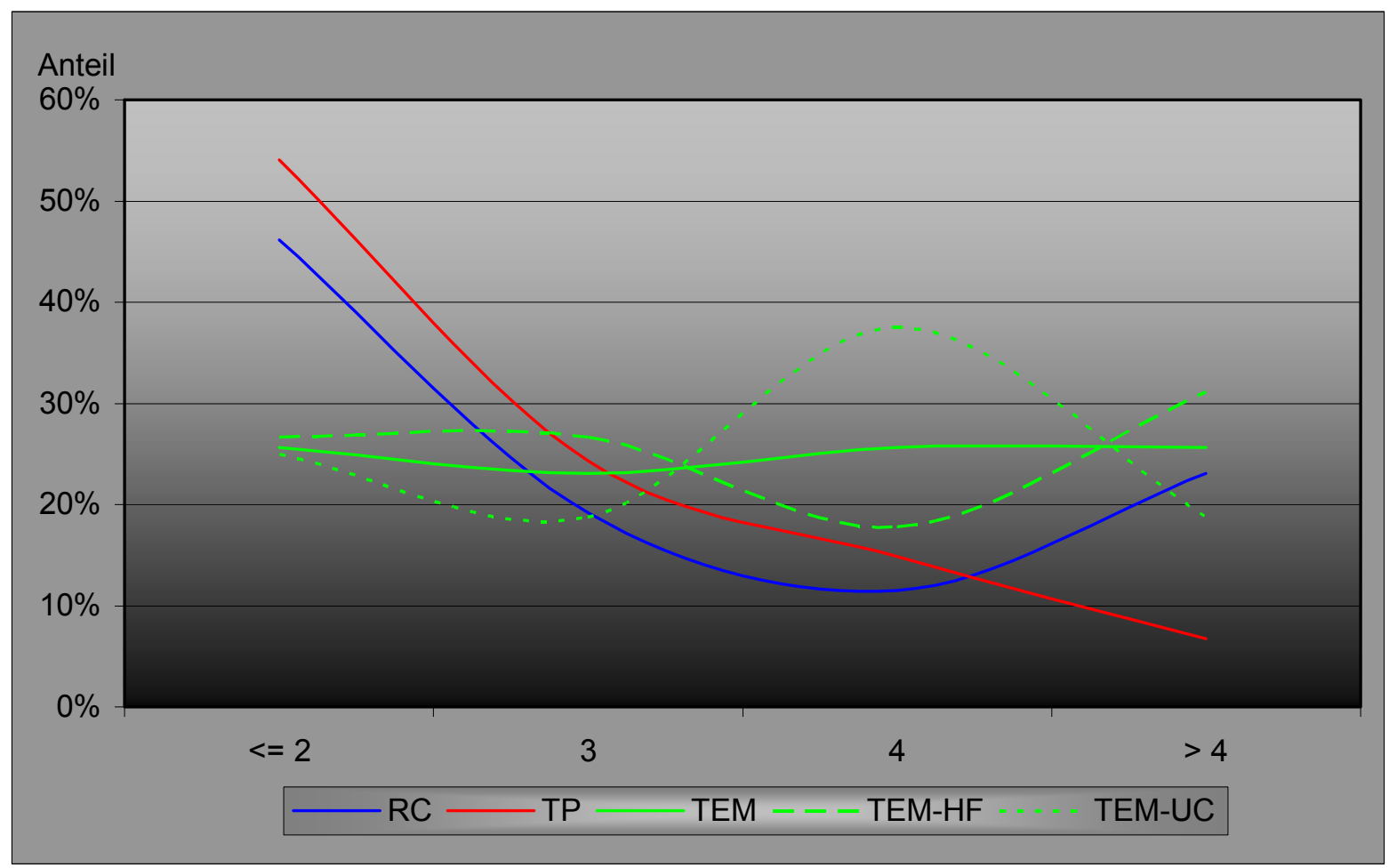

\subsubsection{Höhenlokalisation der Tumore ab ano}

Die Höhenlokalisation der Tumore, gemessen von der Anokutanlinie, wurde den Untersuchungsberichten der Rektoskopie entnommen. In der Gruppe RC waren die Tumore bei 9,5 \pm 3,9 [4 - 20] cm lokalisiert. Für die Gruppe TP wurde 5,7 \pm 2,2 [1 15] $\mathrm{cm}$ als durchschnittliche Höhe berechnet. Innerhalb der Hauptgruppe TEM lagen die Tumore bei 7,0 cm $\pm 2,8$ [4 - 20] cm. Für die Untergruppen TEM-HF und TEM-UC wurden 7,0 $\pm 2,8[4-16] \mathrm{cm}$ und 6,0 $\pm 3,2[4-20] \mathrm{cm}$ bestimmt.

Der Vergleich der absoluten Werte der Höhenlokalisationen zwischen den Hauptgruppen mit dem Wilcoxon-Test zeigte signifikante Unterschiede sowohl für TEM vs. RC ( $p$-Wert =0,009), als auch für TEM vs. TP ( $p$-Wert =0,003). Hierbei lagen die Tumore der Gruppe TEM höher als die von TP und niedriger als die von 
RC. Auch der Vergleich der Untergruppen TEM-HF mit TEM-UC zeigte mit einem pWert von 0,001 einen signifikanten Unterschied, wobei die Tumore der Gruppe TEMHF höher als die der Gruppe TEM-UC lagen.

Bei der Untersuchung der Verteilung der Tumore, kategorisiert nach unterem, mittlerem und oberem Rektumdrittel, die als $\leq 5 \mathrm{~cm},>5-<12 \mathrm{~cm}$ und $\geq 12 \mathrm{~cm}$ definiert wurden, ergaben sich im $\chi^{2}$-Test weder für die Hauptgruppen noch für die Untergruppenvergleiche signifikante Unterschiede.

Die Abbildung 9 zeigt den prozentualen Anteil aller Tumore einer Gruppe bezogen auf die Rektumdrittel.

\section{Abbildung 9: Höhenlokalisation ab ano}

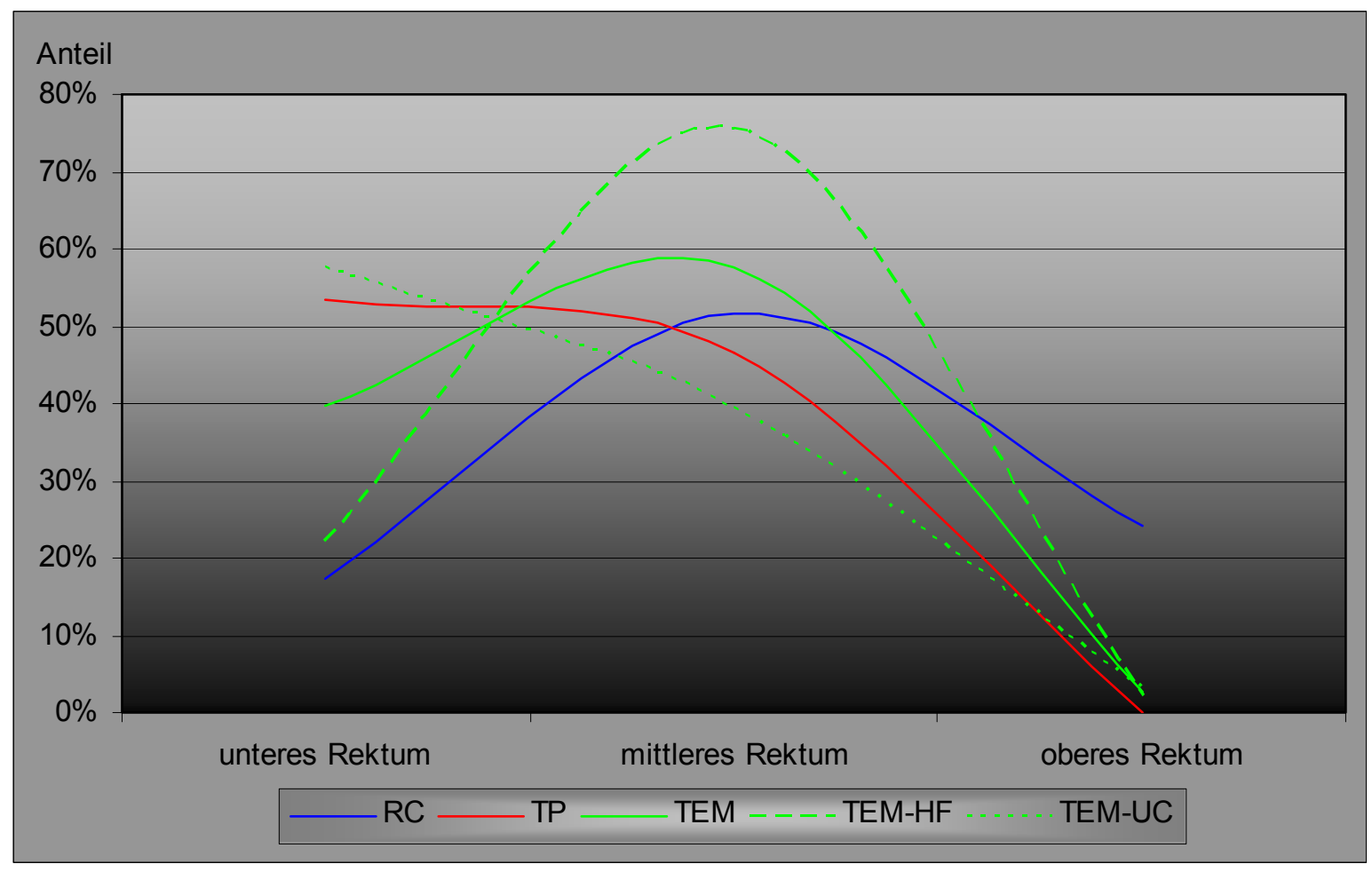

\subsubsection{Tumorlokalisation bezogen auf die Zirkumferenz des Rektums}

Die Verteilung der Tumore in Steinschnittlage wurde in $30^{\circ}$ Schritten untergliedert und im Uhrzeigersinn auf die Summe aller Operationen der jeweiligen Gruppe bezogen betrachtet.

Das Vorliegen oder Fehlen von Tumormasse wurde für jedes $30^{\circ}$-Segment des Rektums mittels des exakten Fisher Tests für die 3 Hauptgruppen verglichen. Hierbei konnte für keines der Segmente ein statistisch signifikanter Unterschied 
nachgewiesen werden. Der Vergleich der Untergruppen TEM-HF vs. TEM-UC erbrachte lediglich in 9 Uhr Steinschnittlage einen signifikanten p-Wert von 0,019.

Die Abbildung 10 zeigt die Verteilung der Tumore auf die Zirkumferenz des Rektums angegeben als den prozentualen Anteil aller Operationen für die 3 Hauptgruppen einzeln sowie für alle Operationsverfahren gemeinsam.

\section{Abbildung 10: Tumorlokalisation bezogen auf die Zirkumferenz des Rektums}

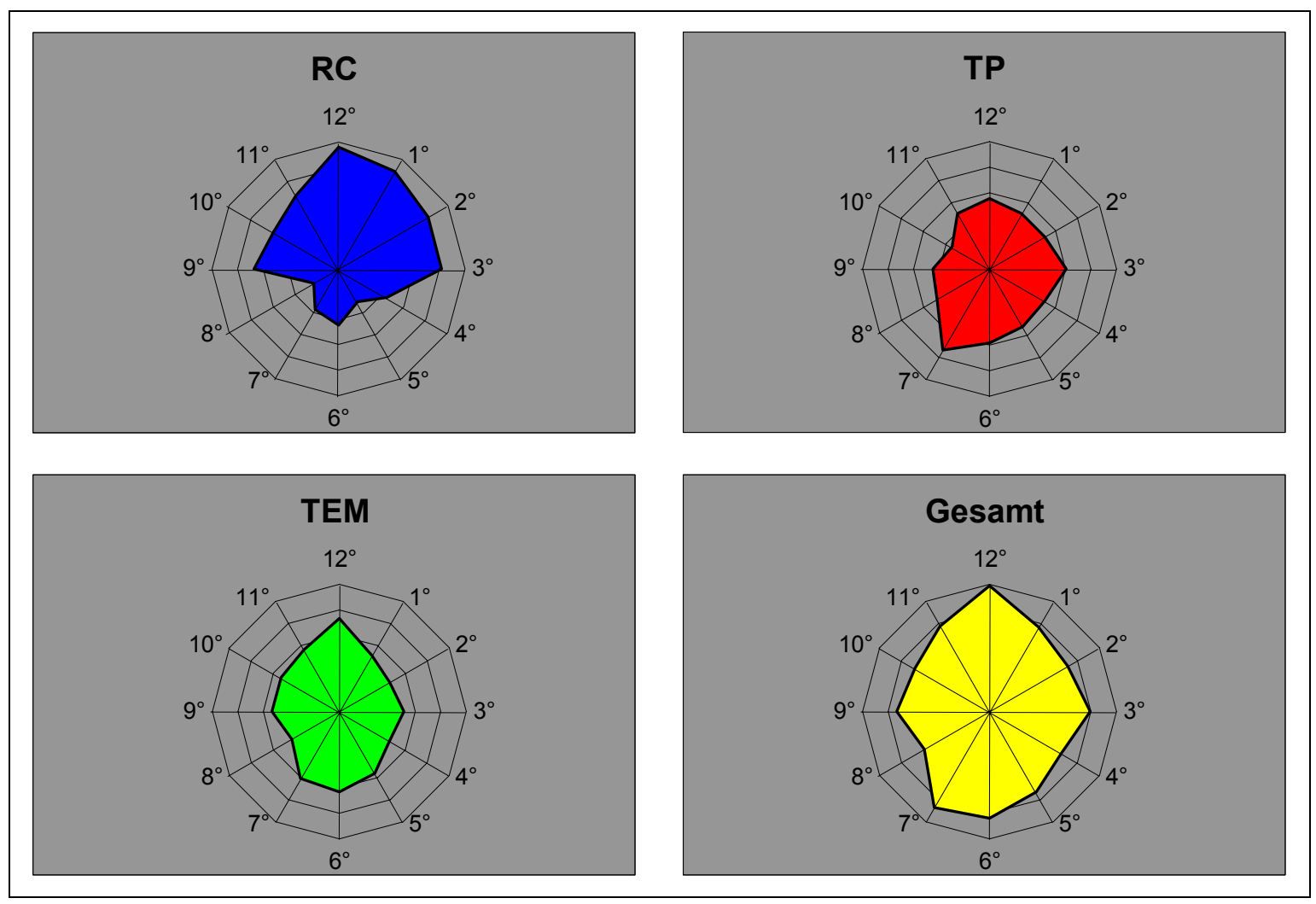

\subsubsection{Residualstatus}

Der Residualstatus wurde den pathologischen Berichten entnommen. Bei Nachweis von Adenombestandteilen in den Resektionsrändern wurde auch bei exzidierten Karzinomen ein R1-Status vermerkt.

Die Verteilung des Residualstatus, bezogen auf die Haupt- und Untergruppen ist, zur besseren Übersicht in absoluten Zahlen und prozentualem Anteil für jede der Gruppen in der Tabelle 12 wiedergegeben. 
Tabelle 12: Residualstatus

\begin{tabular}{|c|c|c|c|}
\hline \multicolumn{4}{|c|}{ Hauptgruppen } \\
\hline & R0 & R1 & $\mathbf{R x}$ \\
\hline RC & $\begin{array}{c}27 \\
100 \%\end{array}$ & $\begin{array}{c}0 \\
0 \%\end{array}$ & $\begin{array}{c}0 \\
0 \%\end{array}$ \\
\hline TP & $\begin{array}{c}36 \\
47,4 \%\end{array}$ & $\begin{array}{c}28 \\
36,8 \%\end{array}$ & $\begin{array}{c}12 \\
15,8 \%\end{array}$ \\
\hline TEM & $\begin{array}{c}60 \\
75,9 \%\end{array}$ & $\begin{array}{c}15 \\
19,0 \%\end{array}$ & $\begin{array}{c}4 \\
5,1 \%\end{array}$ \\
\hline Gesamt & $\begin{array}{c}123 \\
67,6 \%\end{array}$ & $\begin{array}{c}43 \\
23,6 \%\end{array}$ & $\begin{array}{c}16 \\
8,8 \%\end{array}$ \\
\hline
\end{tabular}

\begin{tabular}{cccc}
\hline \multicolumn{4}{c}{ Untergruppen } \\
\hline & R0 & R1 & Rx \\
\hline TEM-HF & 37 & 7 & 1 \\
& $82,2 \%$ & $15,6 \%$ & $2,2 \%$ \\
TEM-UC & 23 & 8 & 3 \\
& $67,7 \%$ & $23,5 \%$ & $8,8 \%$ \\
Gesamt & 60 & 15 & 4 \\
\hline
\end{tabular}

Der $\chi^{2}$-Test zeigte sowohl für den Vergleich der Hauptgruppen TEM vs. TP mit einem p-Wert von 0,001 als auch für den Vergleich TEM vs. RC mit einem p-Wert von 0,019 statistisch signifikante Unterschiede. Die Hauptgruppe TEM liegt damit statistisch nachweisbar mit einem niedrigeren Anteil von fraglich vollständigen $(R x)$ oder sicher unvollständigen (R1) Resektionen zwischen der Gruppe TP und der Gruppe RC. Der Vergleich der Untergruppen TEM-HF und TEM-UC zeigte keinen signifikanten Unterschied.

Die Abbildung 11 stellt die prozentualen Anteile der Resektionsergebnisse Rx, R0, R1 den Operationsverfahren nach gegliedert grafisch dar.

\section{Abbildung 11: Residualstatus}

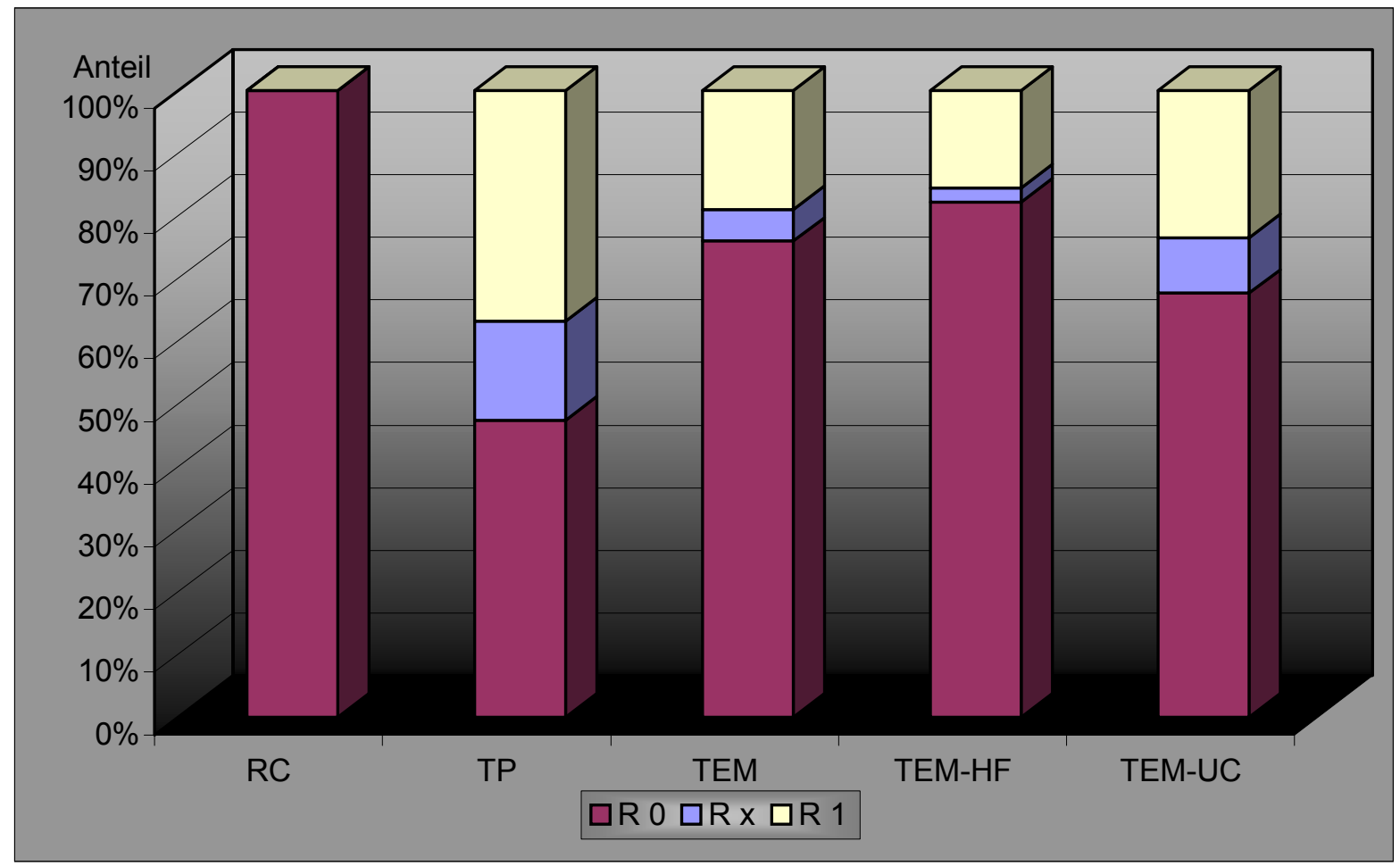


Seit Juni 2000 wurden alle TEM-Präparate direkt nach der Entnahme, noch vor der Fixierung, auf Korkplatten aufgespannt. Von diesem Zeitpunkt an wurde in keinem der 18 Präparate ein Rx-Status diagnostiziert. Es fanden sich jedoch, trotz eines angestrebten Sicherheitsabstandes von $1 \mathrm{~cm}$ in vivo, $3(16,7 \%)$ Resektate, in deren Randbereichen Tumorgewebe nachgewiesen werden konnte. Im Gegensatz zu den nicht aufgespannten Präparaten verminderte sich in dieser Zeit der Anteil Rx und R1 resezierter Tumore von $26,2 \%$ auf $17,7 \%$.

\subsubsection{Nachsorgezeitraum}

Für alle Patienten konnten Nachsorgedaten erhoben werden, jedoch lagen nur für insgesamt $138(85,7 \%)$ der 161 behandelten Patienten den zuvor festgelegten Kriterien (siehe 2.5 Nachuntersuchung und Follow up) entsprechende Daten vor.

Für die Gruppe RC wurde ein Nachsorgezeitraum von 35,1 \pm 30,5 [1 - 135] Monaten berechnet. Der Nachsorgezeitraum der Gruppe TP betrug 32,8 \pm 26,7 [1 - 130] Monate. Bei der Gruppe TEM wurde der Nachsorgezeitraum mit 23,2 \pm 17,5 [1 - 75] Monaten berechnet. Für die Untergruppe TEM-HF wurden 31,6 \pm 18,2 [1 - 75] Monate, für TEM-UC 12,1 \pm 7,4 [1 - 24] Monate berechnet.

Der Vergleich der Hauptgruppen mit dem Wilcoxon-Test zeigte bei TEM vs. TP einen signifikanten Unterschied ( $p$-Wert $=0,025$ ) mit längerem Nachsorgezeitraum für TP. Bei einem p-Wert von 0,098 war ein signifikanter Unterschied zwischen TEM und RC nicht nachzuweisen. Im Vergleich der Untergruppen TEM-HF vs. TEM-UC zeigte der Wilcoxon-Test mit einem p-Wert von 0,001 einen signifikant längeren Nachsorgezeitraum der Untergruppe TEM-HF.

Die Abbildung 12 gibt den prozentualen Anteil der sich je Monat noch in der Nachsorge befindlichen Patienten nach Operationsverfahren gegliedert wieder. 


\section{Abbildung 12: Nachsorgezeitraum}

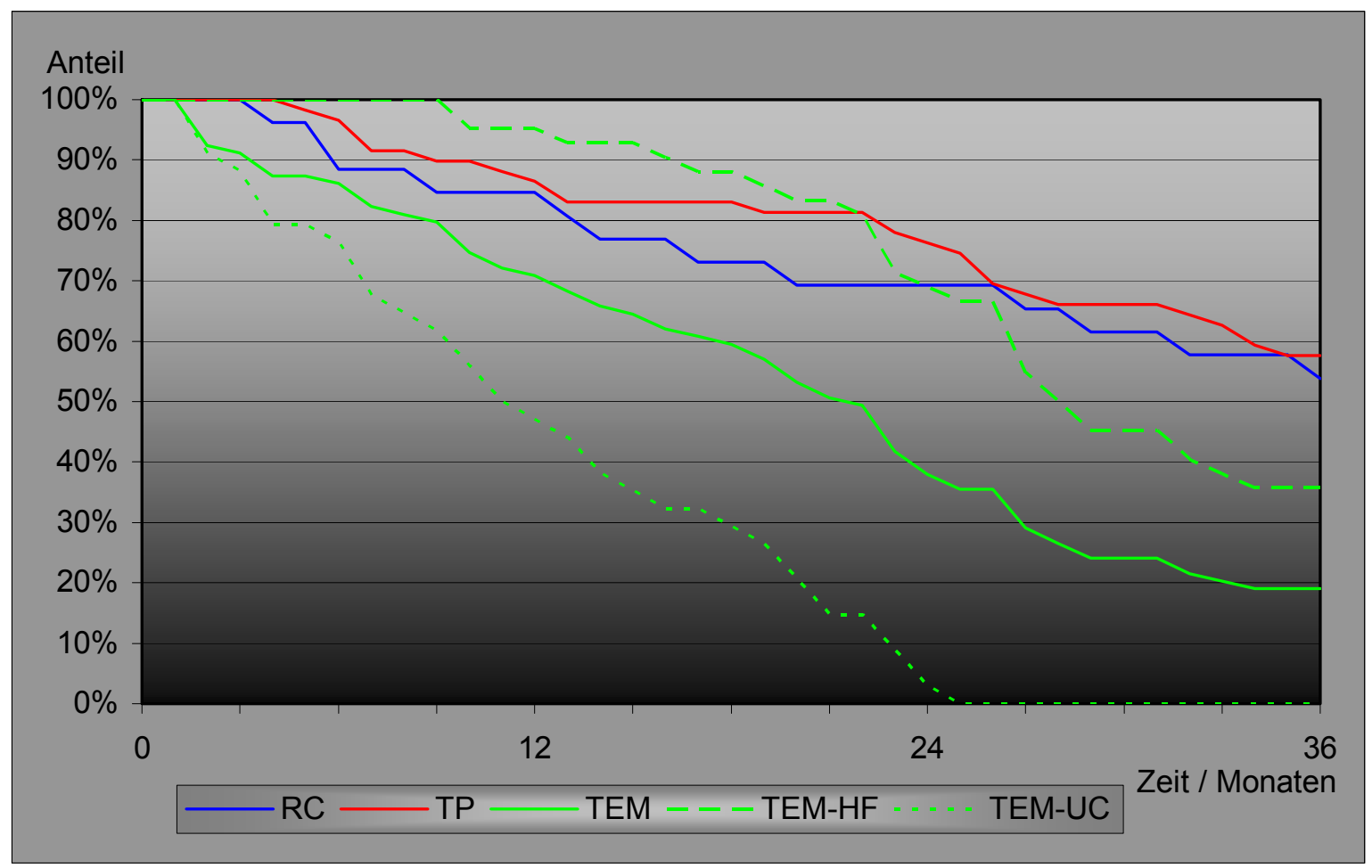

\subsubsection{Zusammenfassung der Vergleichskriterien}

Zusammenfassend zeigt die Untersuchung der Vergleichskriterien einen Unterschied in der Geschlechterverteilung zwischen TEM und TP mit mehr Frauen innerhalb der Gruppe TP. Für das Alter der Patienten zum Zeitpunkt der Operation konnten keine Unterschiede nachgewiesen werden. Die Lokalisation und Größe der resezierten Tumore betreffend konnten für die Gruppe RC insgesamt höher gelegene sowie für die Gruppe TP kleinere Tumore als für TEM nachgewiesen werden. In der Histologie zeigten sich Unterschiede zwischen RC und TEM mit mehr Karzinomen innerhalb der Gruppe RC. Es wurden insgesamt mehr lokale Rezidive in der Gruppe TP reseziert als in der Gruppe TEM. Den Residualstatus betreffend zeigte die Gruppe TEM mehr fraglich vollständige und unvollständige Resektionen als die Gruppe RC, jedoch weniger als die Gruppe TP. Für den Nachsorgezeitraum konnte nur für die Gruppe TP ein längeres Follow up als für die Gruppe TEM nachgewiesen werden. Beim Vergleich der Untergruppen TEM-HF und TEM-UC konnte ein signifikanter Unterschied mit insgesamt längerem Follow up innerhalb der Gruppe TEM-HF nachgewiesen werden. 
Zur besseren Übersicht der Vergleichskriterien sind in der Tabelle 13 alle erhobenen Werte mit Ausnahme der Tumorlokalisation synoptisch dargestellt. Unterschiede zu den im Text beschriebenen Ergebnissen ergeben sich aus der kleineren Rundungsgenauigkeit, die zur Darstellung der Zahlen in einer Tabelle nötig war. Die 3 rechtsseitigen Spalten geben alle Ergebnisse der vergleichenden statistischen Untersuchung an. Zusätzlich sind hierbei die im Text nicht beschriebenen, oberhalb der Signifikanzgrenze liegenden p-Werte angegeben. Signifikante Unterschiede sind in rot hervorgehoben.

Tabelle 13: Zusammenfassung der Vergleichskriterien

\begin{tabular}{|c|c|c|c|c|c|c|c|c|}
\hline & \multirow[b]{2}{*}{ RC } & \multirow[b]{2}{*}{ TP } & \multirow[b]{2}{*}{ TEM } & \multirow[b]{2}{*}{ TEM-HF } & \multicolumn{4}{|c|}{ TEM vs. } \\
\hline & & & & & TEM-UC & $\mathrm{RC}$ & TP & HF vs. UC \\
\hline \multicolumn{9}{|l|}{ Geschlecht } \\
\hline (männlich/weiblich) & $15 / 12$ & $27 / 49$ & $44 / 35$ & $28 / 17$ & $16 / 18$ & 1,000 & 0,016 & 0,253 \\
\hline \multicolumn{9}{|l|}{$\begin{array}{l}\text { Anzahl } \\
\text { Alter }\end{array}$} \\
\hline (Median/Mittelwert \pm Stabw) & $73 / 67 \pm 13$ & $67 / 65 \pm 17$ & $67 / 65 \pm 11$ & $69 / 67 \pm 10$ & $63 / 62 \pm 11$ & 0,332 & 0,308 & 0,057 \\
\hline \multicolumn{9}{|l|}{ in Jahren } \\
\hline $\begin{array}{l}\text { Lage im Rektum } \\
\text { (unteres/mittleres/oberes Rektum) }\end{array}$ & $5 / 15 / 7$ & $39 / 34 / 0$ & $31 / 45 / 2$ & $10 / 34 / 1$ & $21 / 11 / 1$ & 0,002 & 0,956 & 0,748 \\
\hline \multicolumn{9}{|l|}{ Anzahl } \\
\hline (Median/Mittelwert \pm Stabw) & $2,5 / 3,2 \pm 2,8$ & $2 / 2,2 \pm 1,5$ & $3,5 / 3,4 \pm 1,5$ & $3 / 3,4 \pm 1,5$ & $3,5 / 3,4 \pm 1,5$ & 0,262 & 0,001 & 0,922 \\
\hline \multicolumn{9}{|l|}{$\begin{array}{l}\text { in } \mathrm{cm} \\
\text { Art der Tumore }\end{array}$} \\
\hline (Adenom/Karzinom/sonstige) & $8 / 18 / 1$ & $53 / 19 / 4$ & $57 / 20 / 2$ & $31 / 13 / 1$ & $26 / 7 / 1$ & 0,001 & 0,856 & 0,445 \\
\hline \multicolumn{9}{|l|}{$\begin{array}{l}\text { Anzahl } \\
\text { Tumorentstehung }\end{array}$} \\
\hline (Primärtumor/Rezidiv) & $20 / 7$ & $49 / 27$ & $67 / 12$ & $35 / 9$ & $32 / 3$ & 0,248 & 0,005 & 0,216 \\
\hline \multicolumn{9}{|l|}{ Anzahl } \\
\hline $\begin{array}{l}\text { pT Staging } \\
\text { (keine Malignität/pT0(in situ)/pT1) }\end{array}$ & $9 / 3 / 15$ & $54 / 9 / 13$ & $59 / 8 / 12$ & $32 / 5 / 8$ & $27 / 3 / 4$ & 0,001 & 0,877 & 0,691 \\
\hline \multicolumn{9}{|l|}{$\begin{array}{l}\text { Anzahl } \\
\text { Residualstatus }\end{array}$} \\
\hline (Rx/R0/R1) & $0 / 27 / 0$ & $12 / 36 / 28$ & $4 / 60 / 15$ & $1 / 37 / 7$ & $3 / 23 / 8$ & 0,019 & 0,001 & 0,240 \\
\hline \multicolumn{9}{|l|}{$\begin{array}{l}\text { Anzahl } \\
\text { Nachsorgezeitraum }\end{array}$} \\
\hline (Median/Mittelwert \pm Stabw) & $33 / 35 \pm 03$ & $33 / 32 \pm 26$ & $21 / 23 \pm 17$ & $27 / 31 \pm 18$ & $11 / 12 \pm 7$ & 0,098 & 0,025 & 0,001 \\
\hline in Monaten & & & & & & & & \\
\hline
\end{tabular}




\subsection{Zielkriterien}

Im Folgenden werden die untersuchten Zielkriterien

3.3.1 Operationsdauer

3.3.2 Blutverlust

3.3.3 Beatmungszeit

3.3.4 Postoperativer stationärer Aufenthalt

3.3.5 Komplikationen

3.3.6 Rezidive

erläutert.

\subsubsection{Operationsdauer}

Für die Operationsdauer wurde dem Anästhesieprotokoll die Länge der Operation von Schnittsetzung bis Hautverschluss, beziehungsweise vom Einbringen bis Entfernen des Parksspreizers oder des TEM Instrumentariums aufgenommen.

Bezogen auf die 3 Hauptgruppen wurden die kürzesten Operationen mit 53,0 $\pm 29,0$ [10 - 157] min in der Gruppe TP verzeichnet. Mit 178,9 \pm 95,7 min [80 - 495] waren die in der Gruppe RC durchgeführten Operationen die zeitlich aufwendigsten. Die Operationszeiten der Gruppe TEM betrug $104 \pm 52$ [25 - 245], die der Untergruppen TEM-HF und TEM-UC wurden mit 121,4 \pm 55,9 [35 - 245] min beziehungsweise 81,4 $\pm 35,4$ [25 - 180] min berechnet.

Signifikante Unterschiede wurden beim Vergleich mit dem Wilcoxon-Test sowohl für TEM vs. RC ( $p$-Wert $=0,001)$ als auch für TEM vs. TP $(p-$ Wert $=0,001)$ beobachtet. Hierbei lag die durchschnittliche Operationszeit der Hauptgruppe TEM unterhalb der von RC und oberhalb der von TP. Auch der Vergleich der Untergruppen TEM-HF vs. TEM-UC zeigte mit einem p-Wert von 0,001 einen statistisch signifikanten Unterschied mit kürzeren Operationszeiten der Untergruppe TEM-UC.

Die Abbildung 13 gibt den prozentualen Anteil aller Operationen wieder, die bis zur angegebenen Zeit abgeschlossen waren. 


\section{Abbildung 13: Operationsdauer}

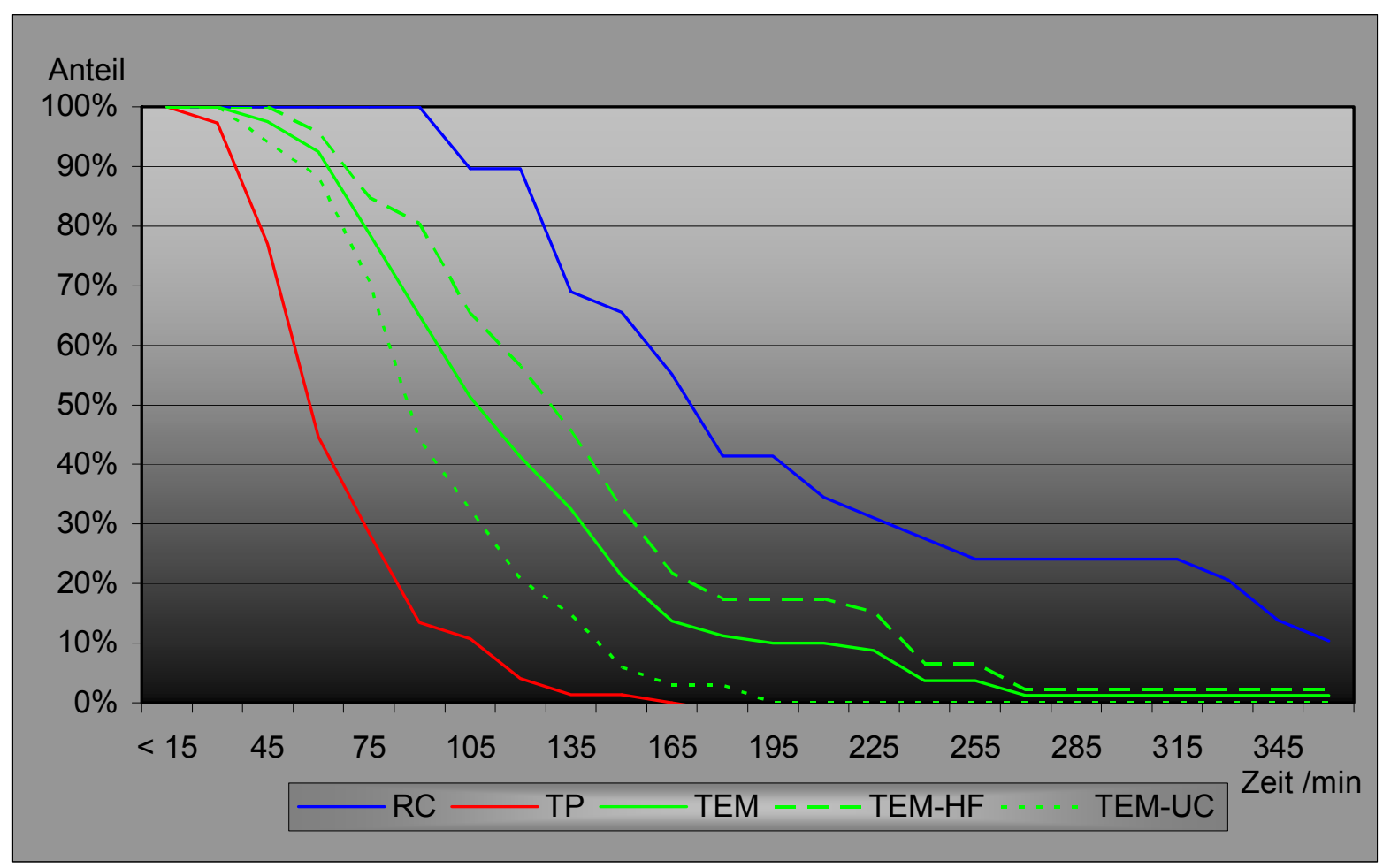

Für die Untersuchung der Operationszeiten der Untergruppen TEM-HF und TEM-UC wurden die Operationen beider Gruppen chronologisch geordnet und zu Gruppen von jeweils 10 zusammengefasst. Für jede dieser Gruppen von 10 Operationen wurde der Mittelwert bestimmt. Die Abbildung 14 gibt diese Mittelwerte grafisch wieder. Um die Anlernphase zur Handhabung des TEM-Instrumentariums zu berücksichtigen, sind hierbei die ersten 10 Operationen der Gruppe TEM linksseitig der Ordinate abgetragen. 


\section{Abbildung 14: Lernkurve TEM}

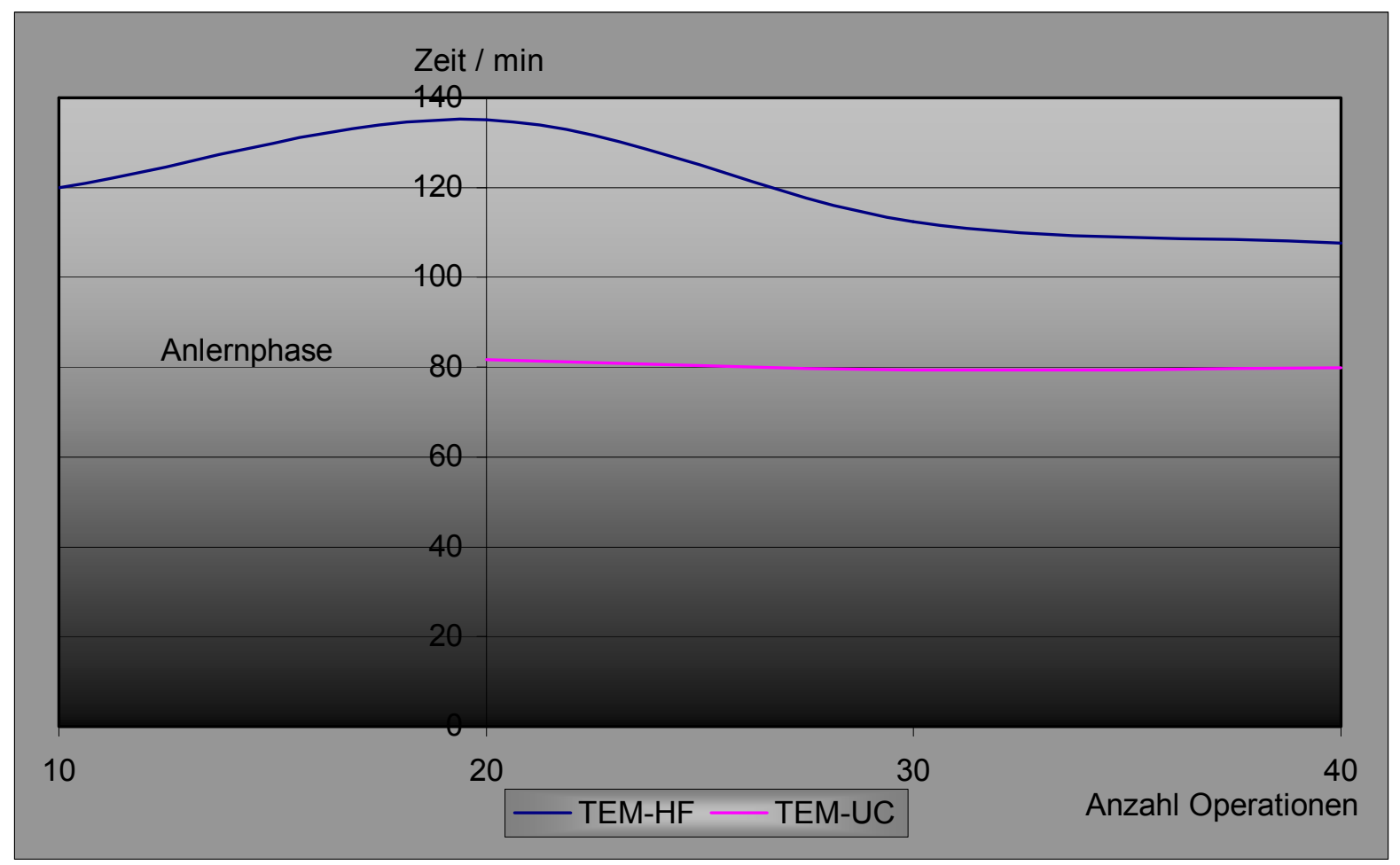

\subsubsection{Blutverlust}

Die Untersuchung des intra- und perioperativen Erythrozytenbedarfs der Operationen wurde den Anästhesieprotokollen sowie der Krankenakte entnommen. Die Tabelle 14 fasst die absolute Anzahl sowie den prozentualen Anteil der intra- und perioperativ substituierten Erythrozytenkonzentrate jedes Operationsverfahrens synoptisch zusammen.

\section{Tabelle 14: Bedarf an Erythrozytenkonzentraten}

\begin{tabular}{ccccc}
\hline \multicolumn{5}{c}{ Hauptgruppen } \\
\hline & $\mathbf{0}$ & $\mathbf{1}$ & $\mathbf{2}$ & $\mathbf{> 2}$ \\
\hline \multirow{2}{*}{ RC } & 16 & 1 & 5 & 5 \\
& $59,3 \%$ & $3,7 \%$ & $62,5 \%$ & $18,5 \%$ \\
TP & 72 & 1 & 3 & 0 \\
& $94,7 \%$ & $1,3 \%$ & $3,9 \%$ & $0 \%$ \\
\multirow{2}{*}{ TEM } & 72 & 7 & 0 & 0 \\
& $91,1 \%$ & 8,9 & $0 \%$ & $0 \%$ \\
\multirow{2}{*}{ Gesamt } & 160 & 9 & 8 & 5 \\
& $87,9 \%$ & $4,9 \%$ & $4,4 \%$ & $2,7 \%$ \\
\hline
\end{tabular}

\begin{tabular}{ccccc}
\hline \multicolumn{5}{c}{ Untergruppen } \\
\hline & $\mathbf{0}$ & $\mathbf{1}$ & $\mathbf{2}$ & $\mathbf{> 2}$ \\
\hline \multirow{2}{*}{ TEM-HF } & 40 & 5 & 0 & 0 \\
& $88,9 \%$ & $11,1 \%$ & $0 \%$ & $0 \%$ \\
TEM-UC & 32 & 2 & 0 & 0 \\
& $94,1 \%$ & $5,9 \%$ & $0 \%$ & $0 \%$ \\
Gesamt & 72 & 7 & 0 & 0 \\
& $91,1 \%$ & $8,9 \%$ & $0 \%$ & $0 \%$ \\
\hline
\end{tabular}

Der Vergleich der Hauptgruppen mit dem Wilcoxon-Test zeigte nur für TEM vs. RC mit einem p-Wert von 0,001 einen signifikanten Unterschied mit mehr 
Erythrozytenkonzentraten in der Gruppe RC. Die Vergleiche TEM vs. TP sowie TEMHF vs. TEM-UC zeigten keine signifikanten Unterschiede.

Die Abbildung 15 gibt den prozentualen Anteil aller Operationen jedes Verfahrens wieder, im Rahmen derer gleich viele oder mehr Erythrozytenkonzentrate substituiert werden mussten.

\section{Abbildung 15: Bedarf an Erythrozytenkonzentraten}

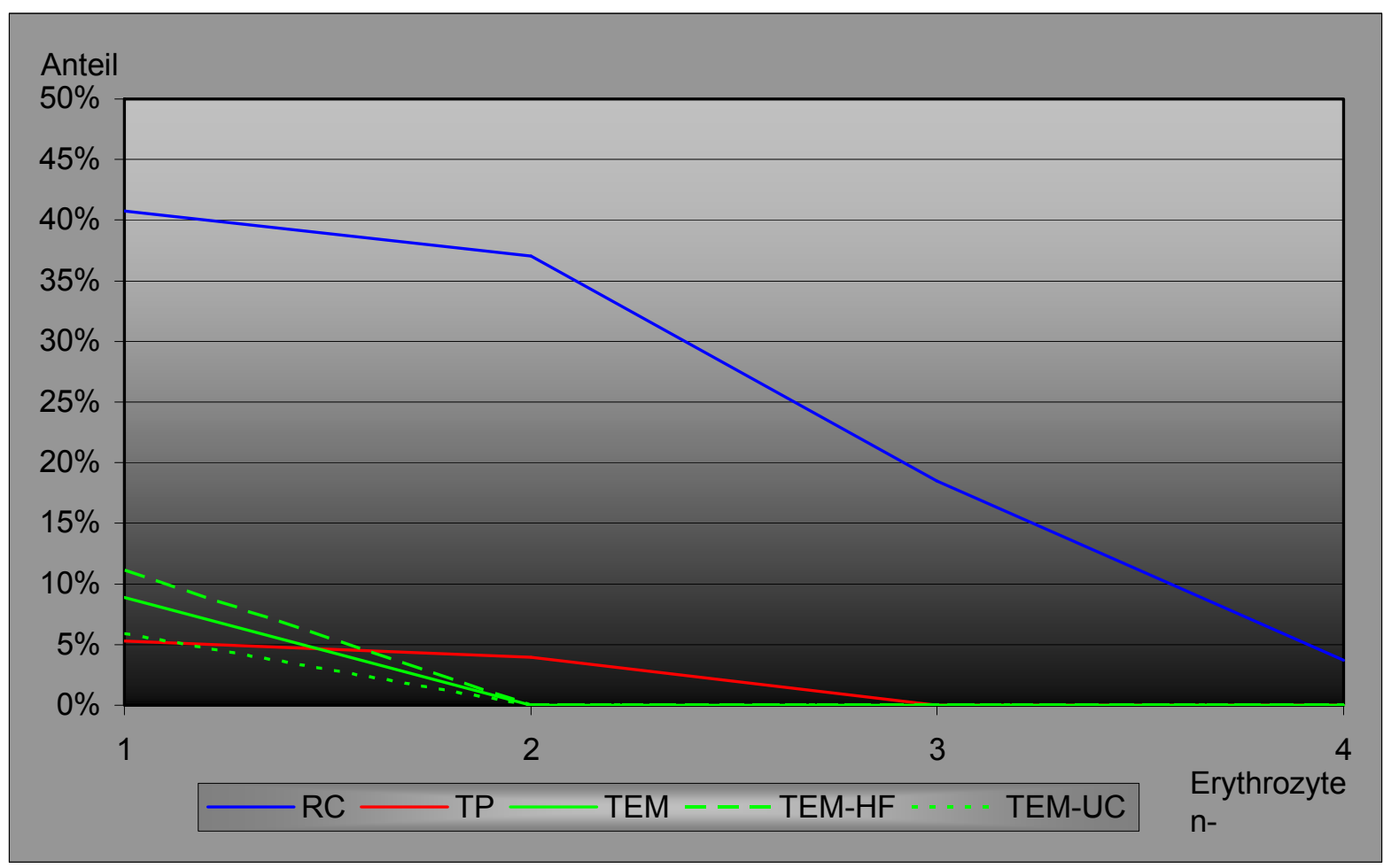

\subsubsection{Beatmungszeit}

Für die Untersuchung der Beatmungszeit wurde der Zeitraum vom Beginn der Beatmung bis zum Zeitpunkt der Extubation aus den Anästhesie- oder Intensivpflegeprotokollen erhoben. Hierbei wurde für die Hauptgruppe RC eine Beatmungszeit von 16,0 \pm 13,8 [2 - 63] Stunden bestimmt. Innerhalb der Hauptgruppe TP wurden Beatmungszeiten von 1,7 \pm 1,4 [1 - 11] Stunden errechnet. Die Beatmungszeiten für TEM wurden mit 3,5 \pm 3,0 [1 - 16] Stunden ermittelt. Die Untergruppen betreffend wurden Beatmungszeiten von 4,4 $\pm 3,6$ [1 - 16] für TEM-HF respektive 2,3 $\pm 1,0[1-5]$ Stunden für TEM-UC ermittelt.

Der Vergleich der Beatmungszeiten mit dem Wilcoxon-Test zeigte sowohl für TEM vs. RC als auch für TEM vs. TP mit p-Werten von jeweils 0,001 signifikante Unterschiede. Hierbei war die durchschnittliche Beatmungszeit von TEM kürzer als 
die von RC, jedoch länger als die von TP. Auch der Vergleich der Untergruppen TEM-HF und TEM-UC zeigte bei einem p-Wert von 0,001 einen signifikanten Unterschied, mit längerer Beatmung von Patienten der Gruppe TEM-HF.

Die Abbildung 16 zeigt den prozentualen Anteil aller Operationen, bei denen die Beatmung des Patienten bis zum angegebenen Zeitpunkt beendet wurde.

\section{Abbildung 16: Beatmungszeit}

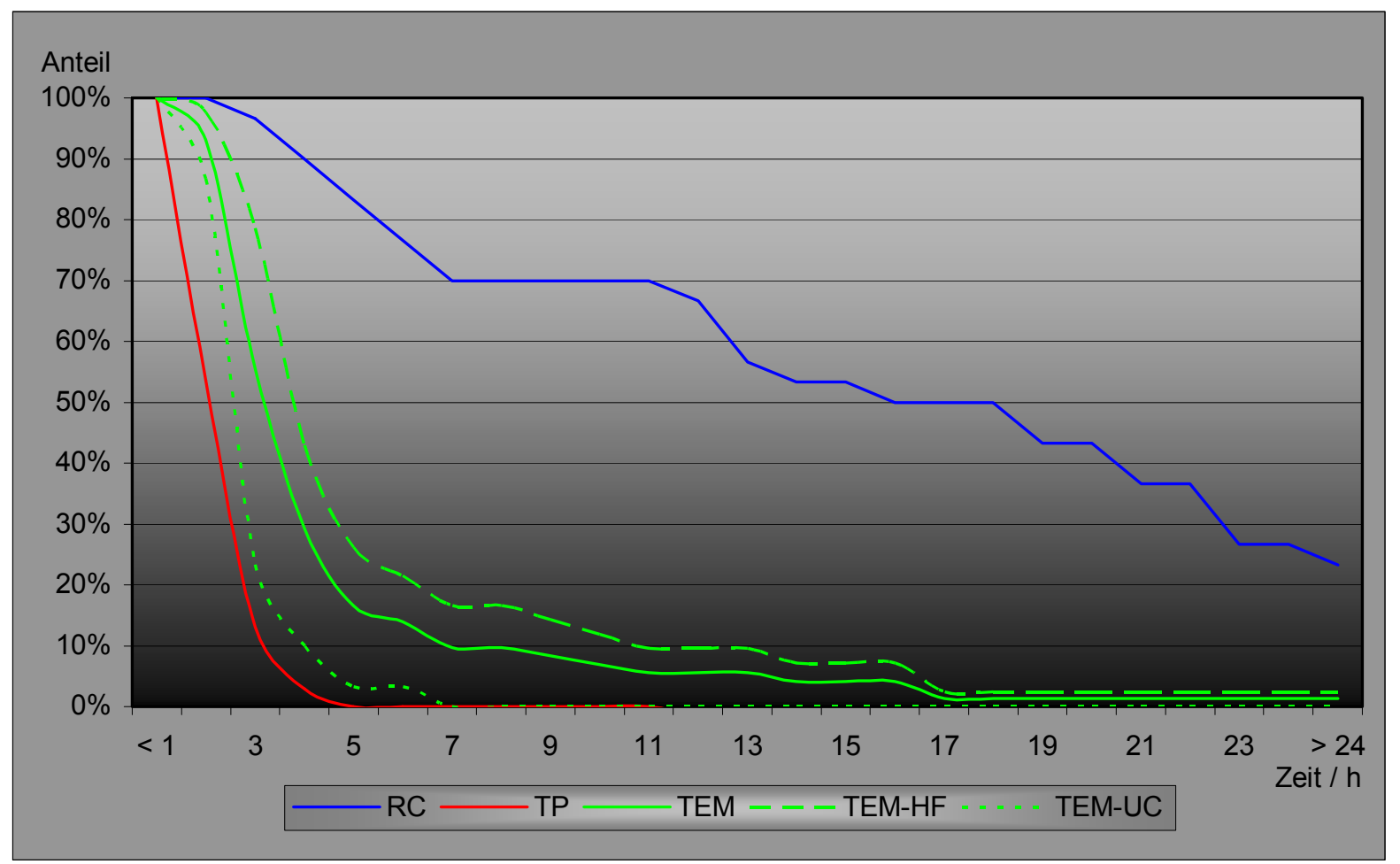

\subsubsection{Postoperativer stationärer Aufenthalt}

Als postoperativer stationärer Aufenthalt wurde der Zeitraum berechnet, der zwischen dem Datum der Operation und dem Tag der Entlassung lag.

Die Untersuchung der Hauptgruppen ergab als höchsten Wert bei der Gruppe RC einen postoperativen stationären Aufenthalt von 14,5 \pm 4,2 [7 - 23]) Tagen. Der kürzeste Aufenthalt wurde für die Gruppe TP mit Zeiten von 7,5 $\pm 3,4$ [1 - 17] Tagen ermittelt. Für die Gruppe TEM wurden Werte von 8,2 \pm 2,3 [3 - 16] Tagen berechnet. Die Untergliederung der TEM zeigte für TEM-HF 8,6 \pm 2,2 [5 - 15] Tage und 7,8 \pm 2,4 [3 - 16] Tage für TEM-UC.

Der Vergleich der Hauptgruppen mit dem Wilcoxon-Test ergab für TEM vs. RC einen signifikanten Unterschied $(p-$ Wert $=0,001)$. Innerhalb der Gruppe TEM konnten 
Patienten hierbei früher entlassen werden. Für die Vergleiche TEM vs. TP und TEMHF vs. TEM-UC konnten keine signifikanten Unterschiede nachgewiesen werden.

Die Abbildung 17 zeigt grafisch den Anteil aller Patienten, die sich bis zum angegebenen postoperativen Zeitpunkt noch in stationärer Behandlung befanden.

\section{Abbildung 17: Postoperativer stationärer Aufenthalt}

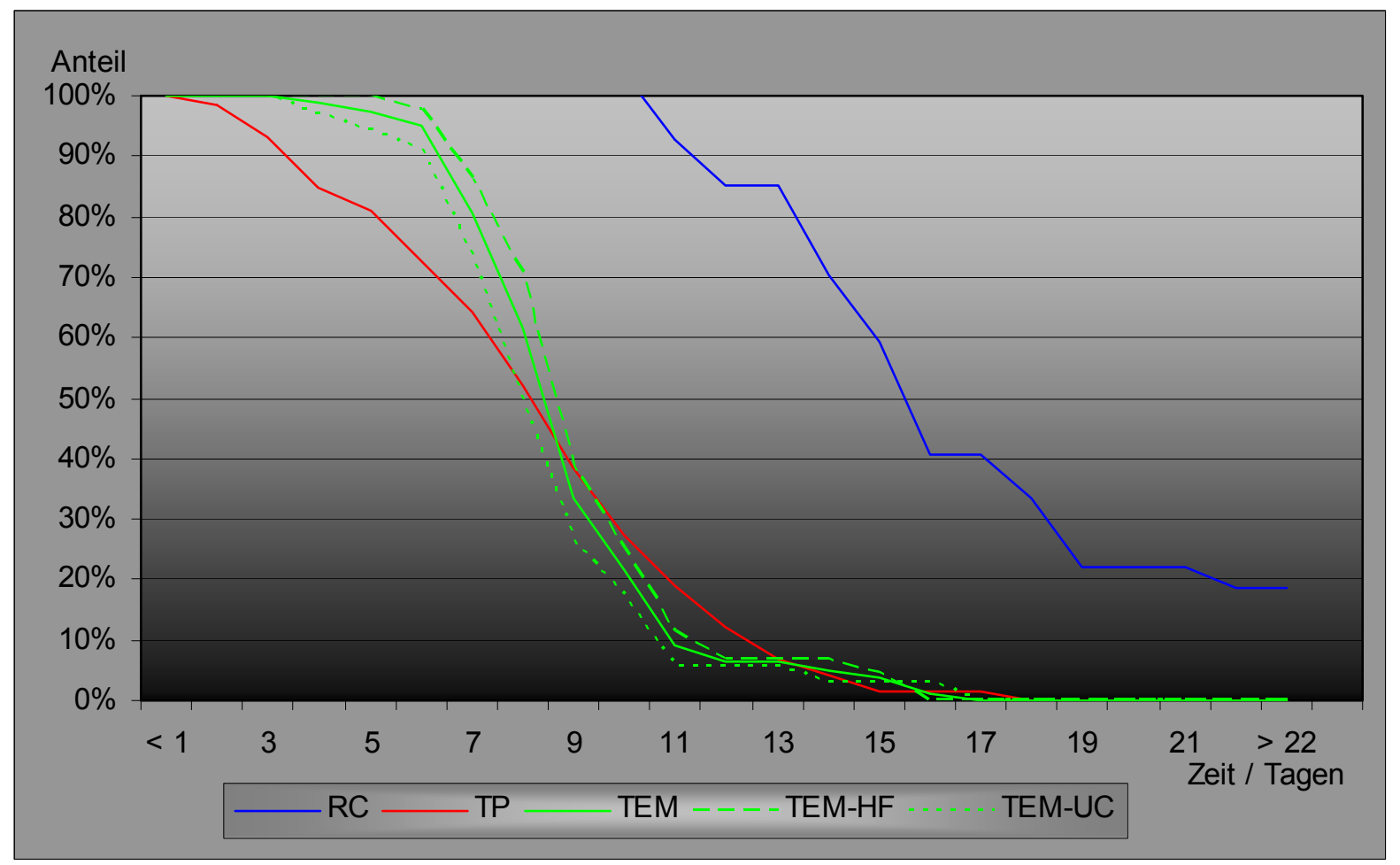

\subsubsection{Komplikationen}

Zur Beurteilung der Komplikationsrate wurden für jede Operation alle Komplikationen erfasst und in Abhängigkeit von der erforderlichen Therapie in konservativ, interventionell und operativ zu behandelnde Komplikationen kategorisiert. Die Ergebnisse dieser Untersuchung sind in Tabelle 15 zusammengefasst.

Tabelle 15: Komplikationen

\begin{tabular}{|c|c|c|c|c|}
\hline \multicolumn{5}{|c|}{ Hauptgruppen } \\
\hline & $\varnothing$ & Kons. & Inter. & OP \\
\hline$R^{*}$ & $\begin{array}{c}14 \\
51,9 \%\end{array}$ & $\begin{array}{c}10 \\
37,0 \%\end{array}$ & $\begin{array}{c}0 \\
0 \%\end{array}$ & $\begin{array}{c}3 \\
11,1 \%\end{array}$ \\
\hline TP & $\begin{array}{c}67 \\
88,2 \%\end{array}$ & $\begin{array}{c}9 \\
11,8 \%\end{array}$ & $\begin{array}{c}0 \\
0 \%\end{array}$ & $\begin{array}{c}0 \\
0 \%\end{array}$ \\
\hline TEM & $\begin{array}{c}73 \\
92,4 \%\end{array}$ & $\begin{array}{c}2 \\
2,5 \%\end{array}$ & $\begin{array}{c}2 \\
2,5 \%\end{array}$ & $\begin{array}{c}2 \\
2,5 \%\end{array}$ \\
\hline Gesamt & $\begin{array}{c}154 \\
84,6 \%\end{array}$ & $\begin{array}{c}21 \\
11,5 \%\end{array}$ & $\begin{array}{c}2 \\
1,1 \%\end{array}$ & $\begin{array}{c}5 \\
2,7 \%\end{array}$ \\
\hline
\end{tabular}

\begin{tabular}{|c|c|c|c|c|}
\hline \multicolumn{5}{|c|}{ Untergruppen } \\
\hline & $\varnothing$ & Kons. & Inter. & OP \\
\hline TEM-HF & $\begin{array}{c}42 \\
93,3 \%\end{array}$ & $\begin{array}{c}0 \\
0 \%\end{array}$ & $\begin{array}{c}1 \\
2,2 \%\end{array}$ & $\begin{array}{c}2 \\
4,4 \%\end{array}$ \\
\hline TEM-UC & $\begin{array}{c}31 \\
91,2 \%\end{array}$ & $\begin{array}{c}2 \\
5,9 \%\end{array}$ & $\begin{array}{c}1 \\
2,9 \%\end{array}$ & $\begin{array}{c}0 \\
0 \%\end{array}$ \\
\hline Gesamt & $\begin{array}{c}73 \\
92,4 \%\end{array}$ & $\begin{array}{c}2 \\
2,5 \%\end{array}$ & $\begin{array}{c}2 \\
2,5 \%\end{array}$ & $\begin{array}{c}2 \\
2,5 \%\end{array}$ \\
\hline
\end{tabular}

* Intra- und perioperative Mortalität 3,7\%

$\varnothing=$ keine Komplikation 
Die intra- und perioperative Letalität während des stationären Aufenthalts betrug bei einem Todesfall in der Gruppe RC 3,7 \%. In den Hauptgruppen TP und TEM trat kein Todesfall auf.

Unter den konservativ beherrschbaren Komplikationen dominierten Nachblutungen, Wundheilungsstörungen sowie Harninkontinenz respektive Harnverhalt mit jeweils 4 Ereignissen. Hierbei traten alle Wundheilungstörungen in der Gruppe RC auf. Darüber hinaus trat bei RC ein Subileus auf, der mit abführenden Maßnahmen behoben werden konnte. In der TEM-HF Gruppe trat in Folge unsachgemäßer Lagerung eine Läsion des N. peroneus auf, die mittels Peroneusschiene versorgt wurde. Darüber hinaus mussten innerhalb der Gruppen TEM-HF und TEM-UC jeweils 1 Nachblutung interventionell rektoskopisch gestillt werden.

Insgesamt wurden 5 revisionspflichtige Operationen beobachtet. Betrachtet man diese im Einzelnen, so zeigten sich in der Gruppe RC 3 revisionspflichtige Komplikationen. Ein Patient verstarb trotz Revision nach anteriorer Rektumresektion unter den Zeichen eines Multiorganversagens. Bei den beiden anderen Fällen handelte es sich um einen subkutanen Abszess, der operativ entlastet werden musste, sowie eine phlegmonöse Appendizitis, die eine Appendektomie erforderlich machte.

In der Gruppe TEM-HF fanden sich 2 revisionspflichtige Komplikationen. Eine hiervon war eine rektovaginale Fistel, die auf dem Boden ausgedehnter Elektrokoagulation zur Blutstillung im Rahmen einer Karzinomresektion auftrat. Bei der neun Monate später zum Fistelverschluss durchgeführten tiefen anterioren Rektumresektion zeigte sich zudem ein pT2, N0, G2 Rezidivtumor. Im zweiten Fall fand sich nach Resektion eines $7 \mathrm{~cm}$ großen Adenoms eine vollständige Nahtdehiszenz, die durch erneute Naht per TEM suffizient verschlossen werden konnte.

Der Vergleich der Hauptgruppen bezüglich der Gesamtkomplikationsrate mit dem exakten Fisher Test zeigte nur für TEM vs. RC einen signifikanten Unterschied mit mehr Komplikationen in der Gruppe RC (p-Wert = 0,001). Auch für TEM-HF vs. TEMUC konnte kein signifikanter Unterschied nachgewiesen werden.

Die Untersuchung der Schwere der Komplikationen wurde mit dem $\chi^{2}$-Test durchgeführt. Auch hier zeigte sich für den Vergleich TEM vs. RC mit einem p-Wert von 0,001 ein signifikanter Unterschied. Darüber hinaus konnte in diesem Test auch 
für TEM vs. TP ein signifikanter Unterschied nachgewiesen werden ( $p$-Wert = 0,034). Demnach wurden in der Gruppe TEM insgesamt weniger Komplikationen beobachtet, diese waren allerdings schwerwiegender und mussten damit revisionspflichtig. Der Vergleich der Untergruppen TEM-HF und TEM-UC zeigte keine Unterschiede.

Der Vergleich der Anzahl komplikationsloser Operationen und solcher mit schweren, operativ revisionspflichtigen Komplikationen zeigte in Fishers exakten Test für RC vs. TEM noch immer signifikante Unterschiede $(p$-Wert $=0,042)$. Im gleichen Design waren sowohl für TP vs. TEM als auch TEM-HF vs. TEM-UC keine Unterschiede mehr nachweisbar.

Zusätzlich zu den aufgetretenen Komplikationen bestand bei 3 Patienten $(11,1 \%)$ der Gruppe RC die Notwendigkeit der Anlage eines Colostomas nach Rektumexstirpation sowie bei 6 (22,2\%) weiteren die der Anlage eines protektiven lleostomas.

Die Abbildung 18 zeigt den prozentualen Anteil aller Operationen, die komplikationslos verliefen bzw. in deren Verlauf Komplikationen konservativ, interventionell oder operativ behandelt werden mussten.

\section{Abbildung 18: Komplikationen}

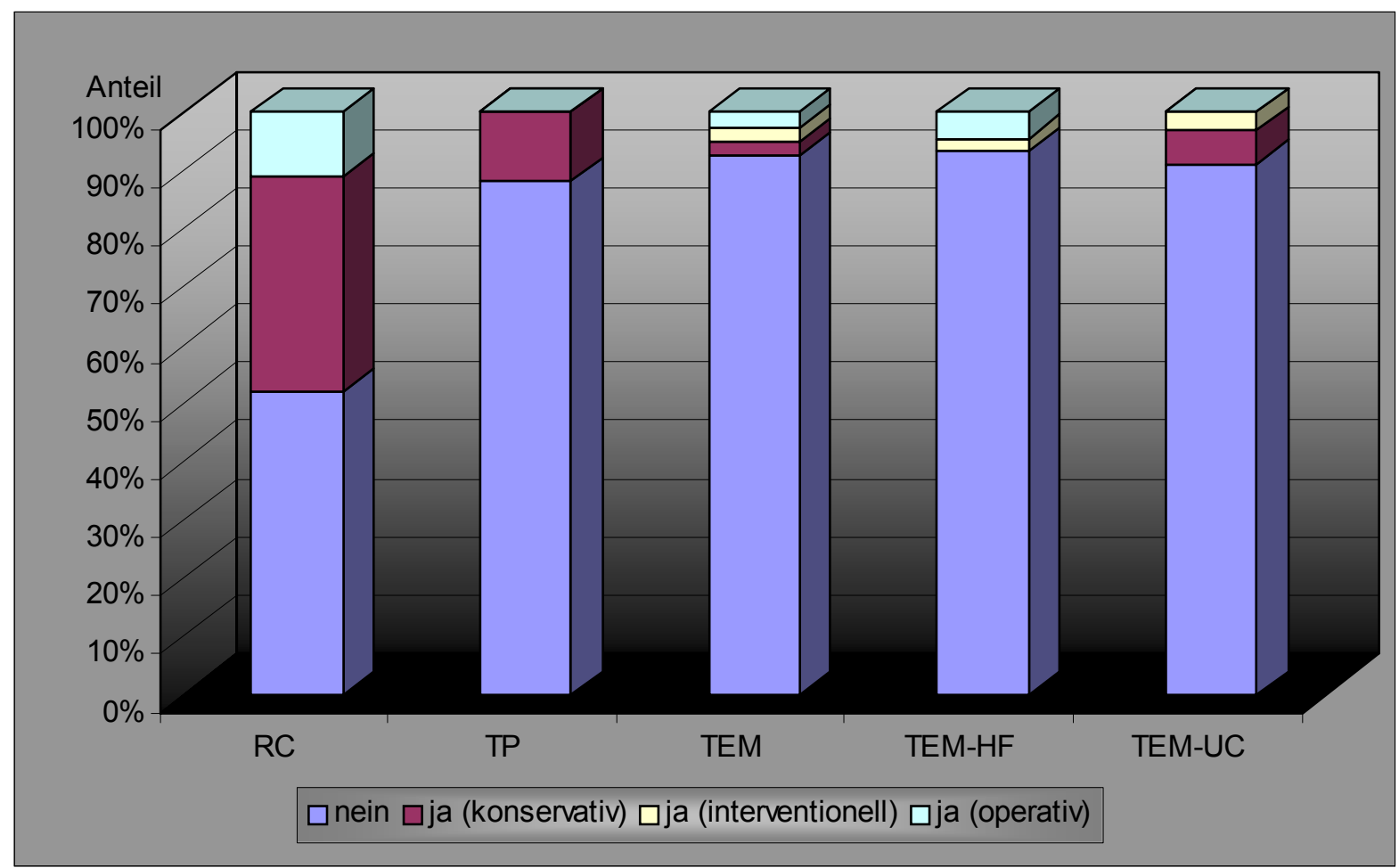




\subsubsection{Rezidive}

Für jede durchgeführte Operation wurde erhoben, ob postoperativ ein Rezidiv auftrat. Im gesamten Kollektiv wurde bis zum Ende des Untersuchungszeitraums kein Fernrezidiv nachgewiesen.

Die Gesamtrezidivquote und -anzahl aller Operationen kann der Tabelle 16 entnommen werden.

\section{Tabelle 16: Rezidive gesamt}

\begin{tabular}{ccc}
\hline \multicolumn{3}{c}{ Hauptgruppen } \\
\hline & $\varnothing$ Rezidiv & Rezidiv \\
\hline \multirow{2}{*}{ RC } & 26 & 1 \\
& $96,3 \%$ & $3,7 \%$ \\
TP & 55 & 20 \\
& $73,3 \%$ & $26,7 \%$ \\
TEM & 72 & 7 \\
& $91,1 \%$ & $8,9 \%$ \\
Gesamt & 153 & 28 \\
& $84,5 \%$ & $15,5 \%$ \\
\hline
\end{tabular}

\begin{tabular}{lcc}
\hline \multicolumn{3}{c}{ Untergruppen } \\
\hline & $\varnothing$ Rezidiv & Rezidiv \\
\hline \multirow{2}{*}{ TEM-HF } & 39 & 6 \\
& $86,7 \%$ & $13,3 \%$ \\
TEM-UC & 33 & 1 \\
& $97,1 \%$ & $2,9 \%$ \\
Gesamt & 72 & 7 \\
\hline
\end{tabular}

Der Vergleich der Hauptgruppen mit dem exakten Fisher Test zeigte nur für TEM vs. TP bei einem p-Wert von 0,042 einen signifikanten Unterschied mit mehr Rezidiven in der Gruppe TP. Wie für TEM vs. RC konnte auch für den Untergruppenvergleich TEM-HF vs. TEM-UC kein signifikanter Unterschied nachgewiesen werden.

Zusätzlich zur Gesamtrezidivquote wurde die Rezidivquote- und Anzahl getrennt nach Adenomen und Karzinomen, denen die Carcinomata in situ zugerechnet wurden, bestimmt. Diese können synoptisch den Tabellen 17 und 18 entnommen werden.

\section{Tabelle 17: Adenomrezidive}

\begin{tabular}{ccc}
\hline \multicolumn{3}{c}{ Hauptgruppen } \\
\hline & $\varnothing$ Rezidiv & Rezidiv \\
\hline \multirow{2}{*}{ RC } & 7 & 1 \\
& $87,5 \%$ & $12,5 \%$ \\
TP & 36 & 17 \\
& $67,9 \%$ & $32,1 \%$ \\
TEM & 52 & 5 \\
& $91,2 \%$ & $8,8 \%$ \\
Gesamt & 95 & 23 \\
& $80,5 \%$ & $19,5 \%$ \\
\hline
\end{tabular}

\begin{tabular}{lcc}
\hline & Untergruppen \\
\hline & $\varnothing$ Rezidiv & Rezidiv \\
\hline \multirow{2}{*}{ TEM-HF } & 27 & 4 \\
& $87,1 \%$ & $12,9 \%$ \\
TEM-UC & 25 & 1 \\
& $96,2 \%$ & $3,8 \%$ \\
Gesamt & 52 & 5 \\
& $91,2 \%$ & $8,8 \%$ \\
\hline
\end{tabular}


Tabelle 18: Karzinomrezidive

\begin{tabular}{ccc}
\hline \multicolumn{3}{c}{ Hauptgruppen } \\
\hline & $\varnothing$ Rezidiv & Rezidiv \\
\hline \multirow{2}{*}{ RC } & 18 & 0 \\
& $100 \%$ & $0 \%$ \\
TP & 16 & 3 \\
& $84,2 \%$ & $15,8 \%$ \\
TEM & 18 & 2 \\
& $90,0 \%$ & $10,0 \%$ \\
Gesamt & 52 & 5 \\
& $91,2 \%$ & $8,8 \%$ \\
\hline
\end{tabular}

\begin{tabular}{lcc}
\hline \multicolumn{3}{c}{ Untergruppen } \\
\hline & $\varnothing$ Rezidiv & Rezidiv \\
\hline TEM-HF & 11 & 2 \\
& $84,6 \%$ & $15,4 \%$ \\
TEM-UC & 7 & 0 \\
& $100 \%$ & $0 \%$ \\
Gesamt & 18 & 2 \\
& $90 \%$ & $10 \%$ \\
\hline
\end{tabular}

Der Vergleich der Untergruppen TEM-HF und TEM-UC zeigte sowohl für Adenome als auch Karzinome weniger Rezidive. Ein signifikanter Unterschied ( $p$-Wert $=0,002$ ) konnte jedoch nur für Adenome erhoben werden.

Die Abbildung 19 zeigt den prozentualen Anteil der postoperativ aufgetretenen Rezidive an der Gesamtheit aller Operationen jeder Gruppe.

\section{Abbildung 19: Rezidive}

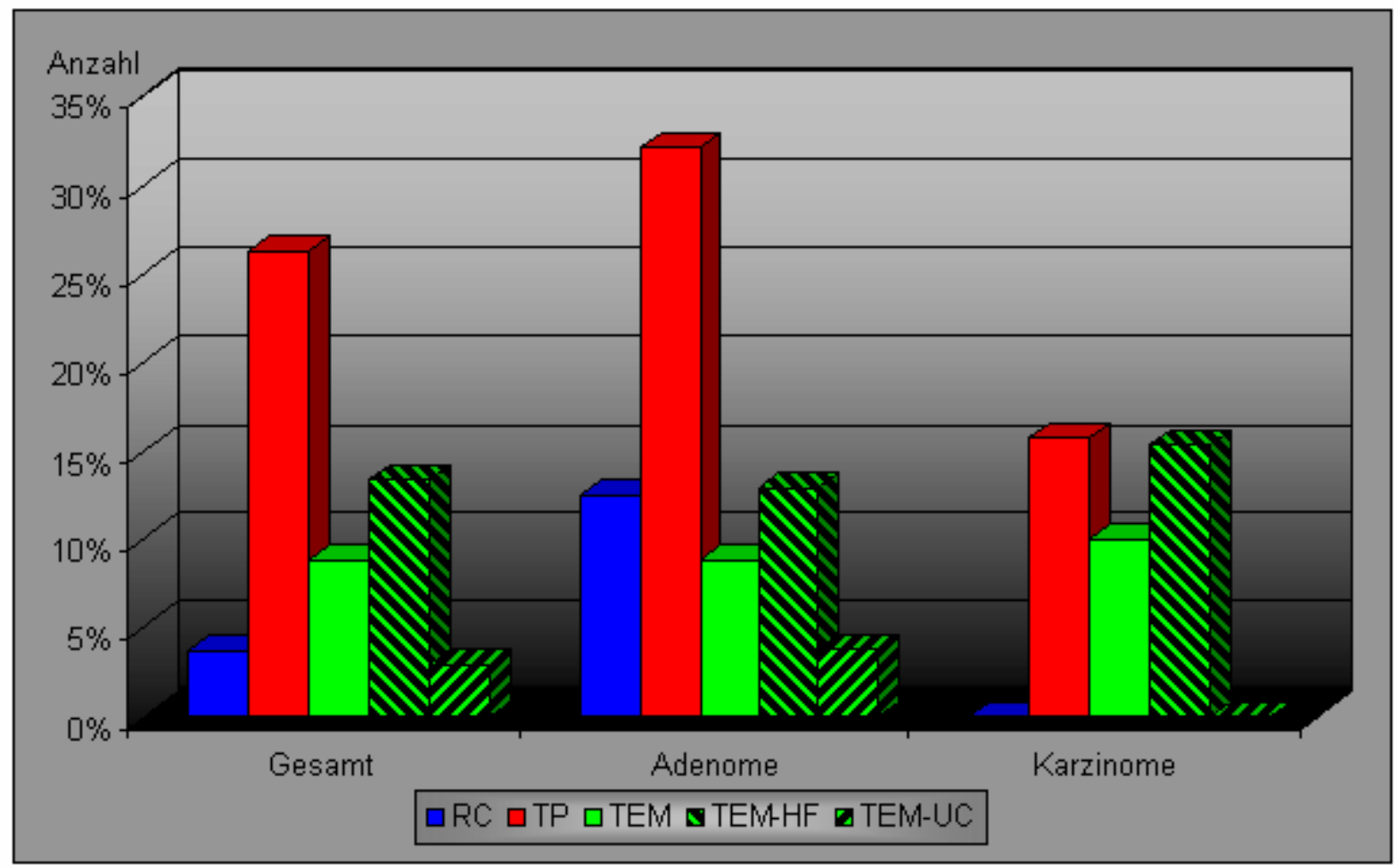

Für alle Rezidive wurde zusätzlich das Intervall zwischen dem Tag der Operation und dessen Auftreten in Tagen erhoben und auf Monate umgerechnet. Für die Hauptgruppe RC wurde eine Rezidivzeit von 17,4 \pm 0 [17,4 - 17,4] Monaten berechnet. Innerhalb der Hauptgruppe TP wurde eine Rezidivzeit von 14,7 $\pm 11,4$ 
[2,5 - 37,5] Monaten beobachtet. Für TEM wurde ein Rezidivzeitraum von 16,3 \pm 11,0 [6,7 - 37,2] Monaten verzeichnet. Die Betrachtung der Untergruppen zeigt für TEM-HF Werte von 16,5 \pm 12,0. [6,7 - 37,2]. In der Untergruppe TEM-UC lag die Rezidivzeit bei $15,1 \pm 0[15,1-15,1]$ Monaten.

Der Vergleich der Hauptgruppen mit dem Wilcoxon-Test zeigte keine signifikanten Unterschiede bezüglich des Zeitpunktes des Auftretens lokaler Rezidive. Auch der Vergleich der Untergruppen TEM-HF und TEM-UC zeigte keinen Unterschied.

Die Abbildung 20 gibt grafisch den prozentualen Anteil der bis zum angegebenen Zeitpunkt aufgetretenen Rezidive an der Gesamtheit aller Rezidive der einzelnen Operationsverfahren für die ersten 36 Monate nach der Operation wieder.

\section{Abbildung 20: Rezidivzeit}

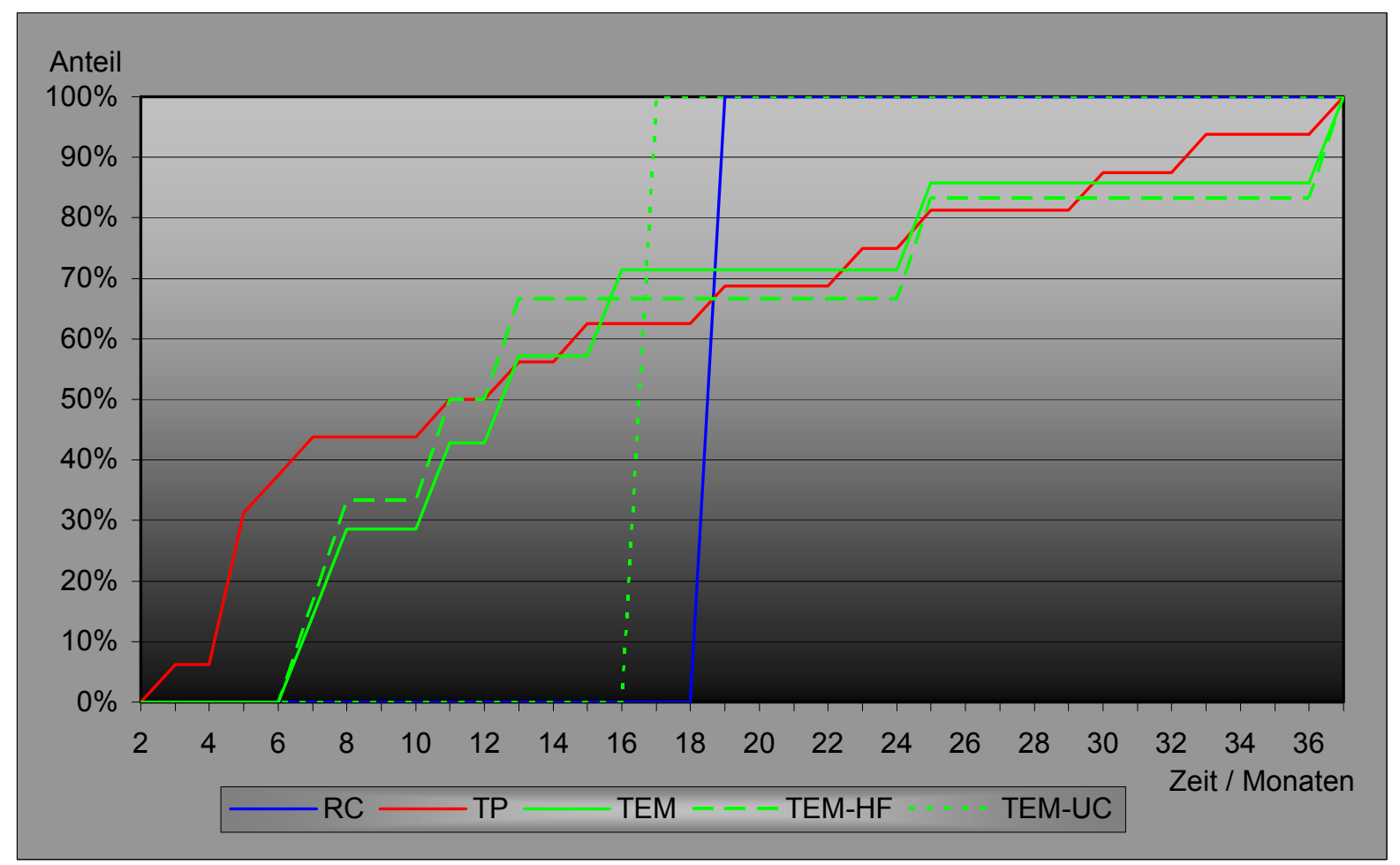

Die genaue Untersuchung der Rezidivfälle erbrachte für die Gruppe RC ein Rezidiv nach R0-Resektion, welches 17 Monate postoperativ mittels TP vollständig entfernt, und histopathologisch als Carcinom in situ beurteilt wurde.

Bei TP traten insgesamt 20 Rezidive, hiervon 17 nach Adenom- und 3 nach Karzinomentfernung auf. Von den 17 Adenomrezidiven lag bei 8 Patienten ein R0Status, bei $3 \mathrm{Rx}$ und bei 6 Fällen R1 vor. Demnach machten Rx- oder R1Resektionen 52,9\% aller Adenomrezidive aus. Insgesamt konnten 13 
Adenomrezidive lokal reseziert werden, die alle erneut Adenome waren. Bei einem unvollständig resezierten Adenom trat nach 6 Monaten erneut ein Adenom auf, welches durch tiefe anteriore Rektumresektion behandelt wurde. Die verbleibenden 3 Patienten wurden auswärts operiert. Weitere Daten zu den histologischen Untersuchungsergebnissen waren hierzu nicht verfügbar.

Von den 3 Rezidiven nach Karzinomresektion der Gruppe TP traten jeweils eines nach R0-, Rx- und R1-Status auf. Bei einem mulitmorbiden Patienten wurde 32 Monate nach R1-Resektion eines Carcinoma in situ ein Rezidiv festgestellt, welches aufgrund der Komorbidität nicht erneut operiert wurde. Nach Rx-Resektion ebenfalls eines Carcinoma in situ konnte 6 Monate später ein Adenomrezidiv vollständig entfernt werden. Über die Therapie des letzten Rezidivs nach R0-Resektion eines pT1-Karzinoms liegen keine weiteren Daten vor.

In der Gruppe TEM wurden insgesamt 7 Rezidive verzeichnet. Hiervon traten 5 nach jeweils vollständiger Entfernung von Adenomen auf, von denen 4 mittels TEM-HF und 1 mittels TEM-UC operiert worden waren. Lokal wurden hiervon 2 mit TEM und 1 mit TP nachoperiert. Histologisch zeigten sich hierbei 3 vollständig entfernte Adenome. Je ein Adenomrezidiv nach TEM-UC und TEM-HF wurden radikal nachoperiert. Das Adenomrezidiv nach TEM-UC trat bei einer Pat. auf, welche bereits 4 Jahre zuvor wegen eines Adenoms mittels anteriorer Rektumresektion operiert worden war. Das 17 Monate später aufgetretene Rerezidiv wurde durch tiefe anteriore Rektumresektion vollständig entfernt. Durch dasselbe Verfahren wurde das 7 Monate nach TEM-HF aufgetretene Adenomrezidiv behandelt. Beide Adenome wurden vollständig entfernt.

In der Gruppe TEM-HF traten nach Entfernung von 2 Karzinomen je 1 Rezidiv nach R0- und R1-Resektion auf. Beide wurden vollständig entfernt. Das Rezidiv nach R0Resektion wurde 8 Monate postoperativ durch abdominoperineale Rektumexstirpation behandelt und zeigte einen pT3-, pN0-, G3-, R0-Status. Das zweite Rezidiv trat nach Resektion eines pT1-Karzinoms auf. Histologisch zeigte sich das Karzinom vollständig entfernt, in einem seitlichen Anschnitt des Präparats konnten jedoch Anteile des präexistierenden Adenoms nachgewiesen werden, so dass der Tumor den eingangs definierten Kriterien nach als unvollständig reseziert gewertet wurde. Das 9 Monate postoperativ aufgetretene Rezidiv wurde mittels tiefer anteriorer Rektumresektion operiert und histopathologisch als vollständig entferntes 
pT2-, pN0-, G2-Karzinom beurteilt. Beide Patienten erhielten postoperativ eine kombinierte Radiochemotherapie.

Alle genannten Patienten blieben im weiteren Verlauf der Nachbeobachtung rezidivfrei.

\subsubsection{Zusammenfassung der Zielkriterien}

Die Operationen der Gruppe TEM wurden in kürzerer Zeit als die von RC, jedoch mit mehr Zeitaufwand als die von TP operiert. Innerhalb der Gruppe TEM konnten die Operationen bei TEM-UC in signifikant kürzerer Zeit durchgeführt werden als bei TEM-HF. In der Gruppe RC wurden mehr Erythrozytenkonzentrate verabreicht als in der TEM-Gruppe, die keinen Unterschied zu TP zeigte. TEM-HF und TEM-UC zeigten keinen Unterschied im Erythrozytenbedarf. Die Beatmungszeit lag bei RC höher als bei TEM, die wiederum höher war als die von TP. Wie für die Operationszeit konnten auch für den Vergleich der Beatmungszeit der Untergruppen kürzere Zeiten für TEM-UC nachgewiesen werden. Bezüglich des postoperativen stationären Aufenthalts war für TEM vs. RC ein signifikanter Unterschied mit längerem Aufenthalt der Patienten der Gruppe RC nachweisbar. Sowohl für TEM vs. TP als auch für TEM-HF vs. TEM-UC konnte kein Unterschied nachgewiesen werden. Quantitativ traten nach RC weniger Rezidive auf als nach TEM, ein statistisch signifikanter Unterschied konnte jedoch nicht nachgewiesen werden. Beim Vergleich TEM vs. TP fanden sich signifikant weniger Rezidive in der Gruppe TEM. TEM-UC zeigte absolut weniger Rezidive als TEM-HF, eine statistische Signifikanz war jedoch auch hier nicht nachweisbar. Der Zeitraum bis zum Auftreten dieser Rezidive zeigte bei keinem der Vergleiche signifikante Unterschiede. Für TEM wurden weniger revisionspflichtige Komplikationen als für RC, für TEM vs. TP konnte dagegen kein Unterschied gezeigt werden. Im Vergleich der Untergruppen TEM-HF und TEM-UC konnte ebenfalls kein Unterschied nachgewiesen werden.

Die Ergebnisse der Untersuchung der Zielvariablen sind in Tabelle 19 synoptisch dargestellt. Die für die Zusammenfassung der Ergebnisse in der Tabelle 19 nötige niedrigere Rundungsgenauigkeit kann zu Abweichungen der Zahlen von denen im Text führen. Die 3 rechtsseitigen Spalten stellen alle Ergebnisse der Inferrenzstatistik dar. Statistisch signifikante $p$-Werte sind hierbei in rot hervorgehoben. 
Tabelle 19: Zusammenfassung der Zielkriterien

\begin{tabular}{|c|c|c|c|c|c|c|c|c|}
\hline & \multirow[b]{2}{*}{ RC } & \multirow[b]{2}{*}{ TP } & \multirow[b]{2}{*}{ TEM } & \multirow[b]{2}{*}{ TEM-HF } & \multicolumn{4}{|c|}{ TEM vs. } \\
\hline & & & & & TEM-UC & RC & TP & HF vs. UC \\
\hline \multicolumn{9}{|l|}{ Operationsdauer } \\
\hline$($ Median/Mittelwert \pm Stabw) & $152 / 178 \pm 95$ & $45 / 53 \pm 29$ & $100 / 104 \pm 52$ & $120 / 121 \pm 56$ & $75 / 81 \pm 35$ & 0,001 & 0,001 & 0,001 \\
\hline \multicolumn{9}{|l|}{$\begin{array}{l}\text { in Minuten } \\
\text { Erythrozytenbedarf }\end{array}$} \\
\hline$(0 / 1 / 2 / 3 / 4)$ & $16 / 1 / 5 / 4 / 1$ & $72 / 1 / 3 / 0 / 0$ & $72 / 7 / 0 / 0 / 0$ & $41 / 5 / 0 / 0 / 0$ & $32 / 2 / 0 / 0 / 0$ & 0,001 & 0,436 & 0,429 \\
\hline \multicolumn{9}{|l|}{ Anzahl } \\
\hline \multicolumn{9}{|l|}{ Beatmungszeit } \\
\hline (Median/Mittelwert \pm Stabw) & $12,5 / 16 \pm 14$ & $1,5 / 1,7 \pm 1,4$ & $3 / 4 \pm 3$ & $3 / 4 \pm 4$ & $2 / 2 \pm 1$ & 0,001 & 0,001 & 0,001 \\
\hline \multirow{2}{*}{\multicolumn{9}{|c|}{$\begin{array}{l}\text { in Stunden } \\
\text { Stationärer Aufenthalt postOP }\end{array}$}} \\
\hline & & & & & & & & \\
\hline (Median/Mittelwert \pm Stabw) & $14 / 14 \pm 4$ & $8 / 7 \pm 3$ & $8 / 8 \pm 2$ & $8 / 9 \pm 2$ & $8 / 8 \pm 2$ & 0,001 & 0,199 & 0,073 \\
\hline \multicolumn{9}{|l|}{ in Tagen } \\
\hline \multicolumn{9}{|l|}{ Entstehung lokaler Rezidive } \\
\hline (kein Rezidiv/Rezidiv) & $26 / 1$ & $56 / 20$ & $72 / 7$ & $39 / 6$ & $33 / 1$ & 0,677 & 0,006 & 0,229 \\
\hline \multirow{2}{*}{\multicolumn{9}{|c|}{$\begin{array}{l}\text { Anzahl } \\
\text { Lokale Rezidive (Adenome) }\end{array}$}} \\
\hline & & & & & & & & \\
\hline (kein Rezidiv/Rezidiv) & $1 / 7$ & $17 / 36$ & $5 / 52$ & $4 / 27$ & $1 / 25$ & 0,406 & 0,002 & 0,195 \\
\hline \multicolumn{9}{|l|}{ Anzahl } \\
\hline \multicolumn{9}{|l|}{ Lokale Rezidive (Karzinome) } \\
\hline (kein Rezidiv/Rezidiv) & $0 / 19$ & $3 / 16$ & $2 / 18$ & $2 / 11$ & $0 / 7$ & 0,256 & 0,320 & 0,411 \\
\hline \multirow{2}{*}{\multicolumn{9}{|c|}{$\begin{array}{l}\text { Anzahl } \\
\text { Zeit bis Rezidivauftreten }\end{array}$}} \\
\hline & & & & & & & & \\
\hline (Median/Mittelwert \pm Stabw) & $17 / 17 \pm 0$ & $11 / 15 \pm 11$ & $13 / 16 \pm 11$ & $11 / 17 \pm 12$ & $15 / 15 \pm 0$ & 0,411 & 0,450 & 0,811 \\
\hline \multicolumn{9}{|l|}{ in Monaten } \\
\hline Komplikationen & & & & & & & & \\
\hline $\begin{array}{l}\text { (keine/konventionell/interventionell/ } \\
\text { operativ) }\end{array}$ & $14 / 10 / 0 / 3$ & $67 / 9 / 0 / 0$ & $73 / 2 / 2 / 2$ & $42 / 0 / 1 / 2$ & $31 / 2 / 1 / 0$ & 0,001 & 0,424 & 1,000 \\
\hline
\end{tabular}

\subsection{Korrelationsanalyse}

\subsubsection{Einflussfaktoren auf die Komplikationsrate}

Zur Untersuchung der Faktoren, die auf die Komplikationsrate Einfluss nehmen, wurden die Operationsdauer, das Geschlecht, die Beatmungszeit, der Erythrozytenbedarf, der Tumordurchmesser und die Höhenlokalisation für die unifaktorielle Untersuchung herangezogen. Hier zeigte nur der Erythrozytenbedarf mit einem p-Wert von 0,007 einen signifikanten Zusammenhang mit der Komplikationsrate. Trotz eines $p$-Wertes von 0,052 wurde die Beatmungszeit in die anschließende multifaktorielle Untersuchung miteinbezogen. In der multifaktoriellen Untersuchung sowohl ohne, als auch mit Wechselwirkung der Faktoren aufeinander, zeigte nur der Erythrozytenbedarf einen signifikanten Zusammenhang mit der Komplikationsrate ( $p$-Wert $=0,030$ und $p$-Wert $=0,009)$. 
In der Tabelle 20 sind alle Ergebnisse der Korrelationsanalyse zusammengefasst. Wechselwirkungen zwischen Faktoren sind für die multifaktorielle Untersuchung durch Sternchen dargestellt.

Tabelle 20: Einflussfaktoren auf die Komplikationsrate

\begin{tabular}{lc}
\hline Komplikationen & $\mathrm{p}=$ \\
\hline unifaktoriell & \\
Operationsdauer & 0,225 \\
Geschlecht & 0,179 \\
Beatmungszeit & 0,052 \\
Erythrozytenbedarf & 0,007 \\
Tumordurchmesser & 0,762 \\
Höhe & 0,365 \\
\hline multifaktoriell ohne Wechselwirkung & \\
Beatmungszeit & 0,190 \\
Erythrozytenbedarf & 0,030 \\
\hline mulitfaktoriell mit Wechselwirkung & \\
Beatmungszeit & 0,066 \\
Erythrozytenbedarf & 0,009 \\
Beatmungszeit * Erythrozytenbedarf & 0,126 \\
\hline
\end{tabular}

\subsubsection{Einflussfaktoren auf den Residualstatus}

Wie für die Einflussfaktoren auf die Entstehung eines Rezidivs wurde für das Resektionsergebnis eine unifaktorielle Untersuchung durchgeführt. Hierfür wurden die Faktoren Tumorentstehung, pT Status, Tumordurchmesser und Höhenlokalisation verwendet. Keiner dieser Faktoren zeigte in der unifaktoriellen Untersuchung einen signifikanten Einfluss auf das Resektionsergebnis.

Die Tabelle 21 fasst die Ergebnisse der unifaktoriellen Untersuchung dieser Faktoren zusammen.

Tabelle 21: Einflussfaktoren auf den Residualstatus

\begin{tabular}{lc}
\hline Residualstatus & $\mathrm{p}=$ \\
\hline Unifaktoriell & \\
Tumorentstehung & 0,761 \\
Grading & 0,182 \\
pT Staging & 0,093 \\
Tumordurchmesser & 0,140 \\
Höhe & 0,138 \\
\hline
\end{tabular}




\subsubsection{Einflussfaktoren auf die Rezidivrate}

Zur Überprüfung eines Zusammenhangs zwischen der Entstehung von Rezidiven und den Faktoren Tumorentstehung, pT Staging, Residualstatus, Tumordurchmesser und Höhenlokalisation des Tumors wurde zunächst eine unifaktorielle Untersuchung durchgeführt.

Hierbei zeigten nur die Tumorentstehung ( $p$-Wert $=0,023)$ und der Tumordurchmesser $(p-$ Wert $=0,024)$ eine Korrelation, der nach die Rezidivquote bei großen und bereits rezidivierten Tumoren ansteigt. Ein Grenzwert für einen sprunghaften Anstieg der Rezidivquote konnte bezüglich der Tumorgröße nicht nachgewiesen werden. In die anschließend durchgeführte multifaktorielle Untersuchung wurde der Residualstatus, trotz eines p-Wert von 0,071 in der unifaktoriellen Untersuchung, einbezogen. Hierbei konnten sowohl bei der Untersuchung ohne als auch mit Wechselwirkung der Faktoren keine signifikanten Zusammenhänge mit der Entstehung eines Rezidivs nachgewiesen werden.

Die Ergebnisse der unifaktoriellen Untersuchung sowie der multifaktoriellen Untersuchung mit und ohne Wechselwirkung der Faktoren sind in der Tabelle 22 synoptisch dargestellt.

Tabelle 22: Einflussfaktoren auf die Rezidivrate

\begin{tabular}{lc}
\hline Rezidiv & $\mathrm{p}=$ \\
\hline unifaktoriell & \\
\hline Tumorentstehung & 0,023 \\
Grading & 0,283 \\
pT Staging & 0,188 \\
Residualstatus & 0,071 \\
Tumordurchmesser & 0,024 \\
Höhe & 0,176 \\
\hline multifaktoriell ohne Wechselwirkung & \\
\hline Tumorentstehung & 0,067 \\
Residualstatus & 0,131 \\
\hline Tumordurchmesser & 0,118 \\
\hline mulitfaktoriell mit Wechselwirkung & \\
\hline Tumorentstehung & 0,632 \\
Residualstatus & 0,336 \\
Tumordurchmesser & 0,442 \\
\hline Tumorentstehung * Residualstatus & 0,671 \\
\hline Tumorentstehung * Tumordurchmesser & 0,969 \\
Residualtumor * Tumordurchmesser & 0,719 \\
\hline Tumorentstehung * Residualstatus * Tumordurchmesser 0,832 \\
\hline
\end{tabular}




\section{Diskussion}

Das Rektumkarzinom gehört, bei steigender Inzidenz, zu den häufigsten malignen Tumoren der westlichen Welt (Nöthinger et Birrer 1999). Aufgrund intensivierter Vorsorgeprogramme und zunehmender Akzeptanz derselben in der Bevölkerung ist auch zukünftig mit einer Zunahme insbesondere der frühen Tumorstadien zu rechnen (Mainprize et al. 1998, Mandel et al. 1999, Midgley et Kerr 1999). Zur chirurgischen Therapie des Rektumkarzinoms stehen verschiedene Operationstechniken zur Verfügung. Goldstandard sind hierbei radikale Verfahren wie die Rektumresektion oder die Rektumexstirpation (RC). Obwohl lokale Operationsverfahren zur Resektion von Rektumtumoren bereits vor mehr als 150 Jahren beschrieben wurden, hat das Therapieprinzip der lokalen Tumorexzision erst in jüngster Zeit einen allgemein akzeptierten Stellenwert im Gesamtkonzept der onkologischen Behandlung lokal begrenzter Rektumkarzinome (pT1) erhalten (Ambacher et al. 1999, Bleday 1997, Schmiegel et al. 2004, Sengupta et Tjandra 2001). Die unterschiedlichen operativen Therapieoptionen bewegen sich dabei in einem Spannungsfeld zwischen kurativem Anspruch mit größtmöglicher onkologischer Radikalität einerseits und dem Ziel eines möglichst guten funktionellen Ergebnisses unter Erhalt der Lebensqualität des Patienten andererseits.

Vor diesem Hintergrund wurde in der vorliegenden Studie dient ein Vergleich des Goldstandard (RC) der Rektumchirurgie mit den zur Zeit gebräuchlichen lokalen Resektionsverfahren, namentlich der konventionellen transanalen Resektion (TP) (Parks 1970) sowie der transanalen endoskopischen Mikrochirurgie (TEM) (Buess et al. 1988a) angestellt. Über die in anderen Publikationen bearbeiteten Fragestellungen hinaus (Buess et Kayser 1996, Chakravarti et al. 1999, Demartines et al. 2001, Winde et al. 1996b), umfasst diese Studie zusätzlich einen Vergleich der Hochfrequenzstromanwendung (HF) mit der Ultraschalldissektionstechnologie (UC) im Rahmen der TEM. Zum Vergleich der genannten Verfahren wurden eine Reihe operationsspezifischer und -unspezifischer Einflußfaktoren auf die Ergebnisqualität hinanalysiert.

Unter den lokalen Operationsverfahren kommt der TEM ein besonderer Stellenwert zu, da mit dieser Technik, ebenso wie mit der RC, nicht nur Tumore des unteren 
Rektumdrittels (0-5 cm ab Anokutanlinie), sondern auch Tumore des mittleren und oberen Rektums (6-16 cm) operiert werden können, die TP technisch nicht zugänglich sind. So beträgt in der vorliegenden Arbeit die durchschnittliche Höhenlokalisation der Tumore ab Anokutanlinie, in Übereinstimmung mit der Literatur, bei TP 5,7 cm (1 cm - $15 \mathrm{~cm}$ ) (Blair et Ellenhorn 2000, Lamont et al. 2000); im Gegensatz zu TEM (Demartines et al. 2001, Palma et al. 2004, Platell et al. 2004) und RC (Mellgren et al. 2000) mit durchschnittlich $7 \mathrm{~cm}$ bzw. 9,5 cm (jeweils $4 \mathrm{~cm}-$ $20 \mathrm{~cm})$.

Darüber hinaus werden mit TEM (Azimuddin et al. 2000, Steele et al. 1996) und der RC (Heintz et al. 1998, Mellgren et al. 2000) erfahrungsgemäß größere Tumore reseziert als mit der TP (Chorost et al. 2001, Taylor et al. 1998). Dementsprechend lagen die Durchschnittswerte des maximalen Tumordurchmessers im ausgewerteten Kollektiv für die TP bei 2,2 cm, gegenüber der TEM und der RC mit 3,4 cm bzw. 3,2 $\mathrm{cm}$.

Der wesentliche Vorteil der lokalen Resektionsverfahren, unter der Voraussetzung gleichwertiger onkologischer Radikalität, liegt jedoch in der geringen Rate an Komplikationen und Nebenwirkungen unter Erhalt der Lebensqualität der Patienten. Während für die konventionelle Rektumchirurgie perioperative Letalitätsraten von 1,0 $\%-4,3 \%$ (Matzel et al. 2004, Sauer et al. 2004), eine Morbidität von ca. $30 \%$ (Sauer et al. 2004) und eine Rate an Stomaanlagen von bis zu 75 \% (Heald et al. 1998) beschrieben sind, betrug in einer deutschen Sammelstatistik von über 1900 TEM-Operationen die Letalität 0,2 \% und die Morbidität 6,3 \%. Stomaanlagen waren in 0,7 \% erforderlich (Salm et al. 1994). Postoperative Einschränkungen der Kontinenzleistung sind nach Rektumresektion bei bis zu 50 \% der Patienten zu erwarten (Renner et al. 1999). Bis zu 40 \% der Patienten klagen über Störungen der Sexual- bzw. Blasenfunktion (Banerjee et al. 1996, Havenga et al. 1996, Junginger 2004). Eine generelle postoperative Einschränkung ihrer Lebensqualität geben bis zu $50 \%$ aller Patienten an (Renner et al. 1999). Demgegenüber konnten prospektiv durchgeführte Funktionsuntersuchungen im Zusammenhang mit TEM-Operationen keine relevanten diesbezüglichen postoperativen Störungen nachweisen (Banerjee et al. 1996).

In Übereinstimmung mit dieser Datenlage wurden in der vorliegenden Studie als Folge des höheren Schweregrades und der größeren Komplexität der RC in dieser 
Gruppe signifikant höhere Komplikationsraten von 48 \%, im Gegensatz zu 12 \% für TP und 8 \% für die TEM verzeichnet. Neben der Häufigkeit der Komplikationen unterschied sich auch der Schweregrad derselben eindeutig: in der RC-Gruppe mussten immerhin 11,1 \% der Patienten einer Revisionsoperation zugeführt werden, während dies nach TEM nur in 2,5 \% der Fälle und nach TP kein Mal erforderlich war. Entsprechend unterschiedlich war auch die perioperative Letalität, die in der RC-Gruppe 3,7 \% betrug, bei TP und TEM dagegen bei $0 \%$ lag.

Einflußgrößen auf die Komplikationsrate, die das wesentlich größere Operationstrauma bei der RC dokumentieren, waren in Übereinstimmung mit anderen Autoren (Neary et al. 2003, Steele et al. 1996, Winde et al. 1996a, Winde et al. 1996b) zum einen erheblich längere Operationszeiten für die RC (179 min vs. 101 min für TEM und 53 min für TP). Zum anderen waren in der RC-Gruppe signifkant häufiger Blutübertragungen von mehr als 2 Erythrozytenkonzentraten erforderlich (RC 18,5 \% vs. TEM und TP mit je 0 \%). Ebenso deutlich waren die Unterschiede hinsichtlich der postoperativ erforderlichen Nachbeatmungszeit auf einer Intensivstation von durchschnittlich 16 Stunden nach RC und 1,7 Stunden nach TP bzw 3,5 Stunden nach TEM. Gerade der Faktor intraoperative Blutung/Erythrozytensubstitutionsbedarf erwies sich in der vorliegenden Arbeit sowohl in der uni- wie der multifaktoriellen Korrelationsanalyse als signifikanter Einflussfaktor auf die Gesamtkomplikationsrate.

Grundlage des Konzeptes der lokalen Operabilität von Rektumtumoren sind Daten vorwiegend retrospektiver Studien, die beim pT1-Rektumkarzinom bezüglich der 5Jahresüberlebensrate keine Unterschiede zwischen lokalen Resektionen, insbesondere der TEM und RC nachweisen konnten (Heintz et al. 1998, Junginger 2004, Matzel et al. 2003). Kritisch anzumerken ist allerdings, dass in diesem Zusammenhang bislang nur wenige prospektiv-randomisierte Studie mit kleiner Fallzahl und begrenztem follow-up vorliegen (Farmer et al. 2002, Meng et al. 2004, Winde 1996 et al. 1996a). In der Studie von Winde et al. wurden vergleichbare Lokalrezidivraten von 4,1 \% bzw. 3,8 \% sowohl nach TEM als auch nach RC von pT1-low-risk- Rektumkarzinomen beschrieben. Die Mehrzahl der Untersuchungsergebnisse anderer Autoren weisen nach TEM Rezidivquoten von ca. $5-10 \%$ auf (Katti 2004, Langer et al. 2001b, Llyod et al. 2002, Maeda et al. 2004, 
Schäfer et al. 2004, Stipa $F$ et al. 2004). Für TP hingegen werden überwiegend hohe Rezidivraten von bis zu 18 \% beschrieben (Mellgren et al. 2000, Paty et al. 2002). Hiermit korrespondierend wurde in der vorliegenden Arbeit eine signifikant niedrigere Rezidivrate von 8,9 \% nach TEM im Vergleich zu 26, 7 \% nach TP gefunden. Nach $\mathrm{RC}$ war die Gesamtrezidivrate mit 3,7 \%, verglichen mit der TEM, am niedrigsten, wenngleich nicht signifikant verschieden. Während in der Gruppe der resezierten Adenome die TEM mit 8,8 \% Rezidivrate im Gegensatz zu 32, 1 \% nach TP und im Vergleich 12, 5 \% in der RC-Gruppe sogar günstiger lag, bleibt kritisch festzustellen, dass in der Gruppe der Karzinome TEM mit immerhin 10 \% Rezidiven zwar deutlich besser abschnitt als TP mit 15,8 \%, aber im Vergleich zu RC mit $0 \%$ doch eine relevante Rate an Rezidiven zu verzeichnen hatte.

Für die Entstehung dieser lokalen Rezidive disponieren laut Schrifttum eine Reihe von Faktoren. Als dominierender Faktor gilt sowohl in klinischen Studien (Bouvet et al. 1999, McDermott et al. 1985, Steele et al. 1996) als auch in Versuchen im Tiermodell (Garcia-Aguilar 2001, Kunihiro et al. 2000) der Residualstatus des Tumors. Dass dieser Zusammenhang hier, wie auch anderorts (Chorost et al. 2001, Manfredi et al. 2001) nicht nachgewiesen wurde, kann neben der niedrigen Fallzahl und damit einer mangelnden Power der statistischen Verfahren auch in der Kürze des Nachbeobachtungszeitraumes begründet liegen. Obwohl der für onkologische Studien geforderte Nachbeobachtungszeitraum von 5 Jahren (Junginger 2004) in der vorliegenden Studie nicht erreicht wurde, konnte die in der Literatur dokumentierte mediane Rezidivzeit von etwa 15 Monaten (RC; Kraemer et al. 2001, Visser et al. 2001, TP; Bouvet et al. 1999, Kim et al. 2001, TEM; Demartines et al. 2001) deutlich überschritten werden, so dass der überwiegende Anteil der Rezidive mutmaßlich bereits erfasst ist. Als weitere Ursache für die Diskrepanz zwischen Residualstatus und Rezidiventwicklung wird diskutiert, dass selbst bei Nachweis von randständigem Tumorgewebe im Resektat, in situ verbliebene Tumoranteile durch thermische oder mechanische Wirkung zerstört worden sein können (Morson 1985). Diese Theorie wird durch eine Studie untermauert, bei der sich in Rektumresektaten, nach vermeintlich unvollständiger lokaler Resektion, in 75\% aller Fälle kein Tumorgewebe mehr nachweisen ließ (Mentges et al. 1997).

Neben dem Residualstatus des Tumors sind die Faktoren Tumorgröße (Jørgensen et al. 1993) und Art des operierten Tumors im Sinne von Rezidiv oder Primärtumor 
(Winde et al. 1996b) hinsichtlich des Rezidivrisikos relevant. Beide Prognoseparameter konnten in der hier durchgeführten Korrelationsanalyse ebenfalls bestätigt werden.

Bezüglich der Ökonomie, einem weitereren Aspekt, der beim Vergleich unterschiedlicher Operationsverfahren aktuell und zukünftig vermehrt zur Diskussion stehen wird, ist die postoperative stationäre Verweildauer von besonderem Interesse. Hier zeigt sich, wie bereits von anderen Autoren beschrieben (Arribas del Amo et al. 2000, Benoist et al. 2001, Buess et al. 1988b, Saclarides 2004, Winde et al. 1996b) ein eindeutiger Vorteil für die lokalen Verfahren mit durchschnittlich je 8 Tagen für TEM und TP gegenüber RC mit 14,5 Tagen. Einer amerikanischen Untersuchung folgend ergibt sich aus einer solchen Verkürzung der stationären Verweildauer durch lokale Operationsverfahren eine Reduktion der Therapiekosten um fast $75 \%$ im Vergleich zur RC (Cocilovo et al. 2003).

Ein weiterer Aspekt der vorliegenden Arbeit betraf den Vergleich der Ergebnisse der TEM durch technische Modifikation, nämlich den Ersatz der Hochfrequenzstromtechnik durch die Ultraschalldissektionstechnologie.

Als ein Kritikpunkt an der TEM-Technik gilt neben hohen Anschaffungskosten der hohe Anspruch, der an die technischen Fertigkeiten des Operateurs gestellt wird. Dieser erfordert umfangreiche Erfahrungen mit der Handhabung des Instrumentariums und ist mit einer entsprechend flachen Lernkurve vergesellschaftet (Richter et al. 1996). In der vorliegenden Studie konnten in der TEM-HF-Gruppe mit zunehmender Erfahrung des Operateurs eine Reduktion der durchschnittlichen Operationszeit um 11\% dokumentiert werden. Dieser Lerneffekt sinkt ab der zwanzigsten Operation auf unter $3 \%$ und kann von dort an als ausgeschöpft betrachtet werden. Der Vergleich der Operationszeiten von TEM-HF und TEM-UC zeigt jedoch, dass selbst ein mit mehr als 20 durchgeführten Eingriffen als erfahren geltender TEM-Chirurg mit der Hochfrequenzstromtechnik im Mittel $35 \%$ mehr Zeit benötigt, als mit der Ultraschalltechnik. Diese signifikant kürzeren Operationszeiten für TEM-UC von durchschnittlich 81, 4 min im Vergleich zu TEM-HF mit 121,4 min, die durch Daten anderer Arbeitsgruppen bestätigt werden (Lirici et al. 2003), erklären sich durch eine optimierte Übersichtlichkeit im Operationsgebiet (Kanaya et al. 1998, Langer et al. 2001b). Ursächlich dafür ist die Vermeidung von intraoperativen Blutungen durch die UC-Technik, wodurch Koagulationen vermieden werden. Diese 
zwingen nämlich ihrerseits durch störende Rauchentwicklung zum zeitaufwendigen Absaugen des Rauches mit häufiger Notwendigkeit einer Neujustierung des Instrumentariums und beeinträchtigen darüber hinaus die, für eine subtile Operationstechnik erforderliche, beständig optimale Sicht des Operateurs.

$\mathrm{Ob}$, und wie weit durch Einsatz der Ultraschalldissektionstechnik auch die Komplikationsrate und Rezidivquote im Rahmen der TEM gesenkt werden kann, war in der vorliegenden Studie nicht abschließend beurteilbar. So fanden sich für TEMUC und TEM-HF mit 8,8 \% bzw. 6,7 \% zwar insgesamt vergleichbare Komplikationsraten, allerdings traten nur in der TEM-HF-Gruppe in $4 \%$ der Fälle operativ revisionspflichtige Komplikationen auf. Signifikante Unterschiede konnten hierbei jedoch nicht nachgewiesen werden. Ähnlich verhält es sich mit der Rezidivquote. Auch hier konnten keine signifikanten Unterschiede nachgewiesen werden, wenngleich die Rezidivrate für HF im Vergleich zu UC höher lag: 13, 3 \% vs. $2,9 \%$ insgesamt, $12,9 \%$ vs. $3,8 \%$ für die Adenome und $15,4 \%$ vs. $0 \%$ für die Karzinome. Unter dem Eindruck des relativ kleinen Kollektivs und noch kurzen Nachbeobachtungszeiträumen sind verlässliche Aussagen derzeit jedoch noch nicht möglich.

Insbesondere in den USA besteht derzeit ein Trend, die Ergebnisse nach lokaler Operation von Rektumkarzinomen mit Hilfe adjuvanter Radio/Chemotherapiekonzepte zu verbessern. Einzelne Arbeitsgruppen berichten nach lokaler Tumorexzision von pT1-Tumoren und adjuvanter Radio-/Chemotherapie über eine deutliche Reduktion der lokalen Rezidivrate (von $24 \%$ auf 0 \%) oder eine Steigerung der 5-Jahresüberlebensrate (von $37 \%$ auf $85 \%$ ) (Lamont et al. 2000, Chakravarti et al. 1999). Allerdings sind diese Angaben aufgrund geringer Fallzahlen und unzureichender Studiendesigns bislang wenig überzeugend. Auch in der größten publizierten Übersichtsarbeit, die Daten von 14 Studien der englischsprachigen Literatur zu diesem Thema einschließt, ergaben sich für das pT1-Karzinom keine Unterschiede bezüglich der Rezidivquote nach vollständiger lokaler Exzision ohne $(9,7 \%)$ bzw. mit $(9,5 \%)$ adjuvanter Radio-/Chemotherapie (Sengupta et Tjandra 2001).

Unterdessen haben erste Arbeitsgruppen die Indikation zu kombinierten Therapiekonzepten (lokale Exzision + Radio-/Chemotherapie) sogar auf höhere 
Diskussion

Tumorstadien (pT1-high-risk, pT2 und pT3) ausgedehnt (Chakravarti et al. 1999, Lezoche et al. 2002, Ruo et al. 2002, Steele et al. 1996). Obwohl die Datenlage begrenzt ist und wiederum keine kontrollierten Studien vorliegen, sind die ersten Ergebnisse bezüglich adjuvanter Radio-/Chemotherapie nach kurativer lokaler Exzision von pT2- undpT3-Tumoren, mit Rezidivraten von bis zu 33 \% ernüchternd (Ruo et al. 2002, Steele et al. 1996).

Vielversprechender sind erste Ergebnisse bei pT2- und pT3-Rektumkarzinomen innerhalb neoadjuvanter Radio-/Chemotherapiekonzepte. Zwar ist auch hier die Datenlage spärlich, doch wurde in einer Studie an vorbehandelten pT2- und pT3Tumoren, die sich unter der Radio-/Chemotherapie komplett zurückgebildet hatten (Complete Response) und nachfolgend lokal reseziert wurden, nach einem follow-up von 24 Monaten kein Rezidiv nachgewiesen (Kim et al. 2001). In einer größeren Vergleichsstudie an 431 pT3-Rektumkarzinomen erfolgte nach neoadjuvanter Radio/Chemotherapie bei 26 Patienten eine lokale Exzision, 405 Patienten wurden konventionell radikal operiert. Nach einer durchschnittlichen Nachbeobachtung von 46 Monaten lagen die Rezidiv- und die 5-Jahresüberlebensrate bei $6 \%$ bzw. $86 \%$ in der lokal-chirurgischen Gruppe und bei $8 \%$ bzw. $81 \%$ in der radikal-chirurgischen (Bonnen et al. 2004).

Aufgrund der aktuellen Datenlage kann aus onkologischer Sicht eine Ausweitung der strengen Indikationskriterien für eine lokale chirurgische Therapie von Adenokarzinomen des Rektums derzeit nicht empfohlen werden (Schmiegel et al. 2004). Diese umfassen in kurativer Intention, neben Adenomen, ausschließlich Carcinomata in situ (pTis) und pT1-,low-risk"-Karzinome (G1-2, L0, R0, $\leq 3 \mathrm{~cm}$ ). Vor jeder Einführung adjuvanter oder neoadjuvanter Therapiekonzepte bzw. der Einbeziehung höherer Tumorstadien oder "high-risk"-Karzinomen bedarf es der Überprüfung dieser Konzepte in kontrollierten Studien. Am ehesten dürften hier von einem neoadjuvanten Therapiekonzept Fortschritte zu erwarten sein.

Besteht eine Indikation zur lokal-chirurgischen Therapie, stellt die TEM die Methode der Wahl dar. Aufgrund der hier vorliegenden Daten scheint die Ultraschalldissektionstechnik die Ergebnisse TEM weiter verbessern zu können. Zur abschließenden Beurteilung ist es jedoch wünschenswert, die hier erhobenen 
Befunde im Rahmen einer prospektiv-randomisierten und, in Anbetracht der geringen Inzidenz der in Frage kommenden Tumorentität, multizentrisch geplanten Studie zu überprüfen. 


\section{$5 \quad$ Zusammenfassung}

Die Ergebnisse dieser Studie zeigen, dass der Einsatz der transanalen endoskopischen Mikrochirurgie eine sinnvolle Alternative zur radikalchirurgischen Therapie gutartiger Tumore und früher so genannter pT1-low-risk-Karzinome darstellen kann. Bei vergleichbarer onkologischer Radikalität mit vergleichbaren Rezidivquoten zeichnet sich dieses Verfahren durch eine niedrigere Morbidität und Letalität sowie höhere Lebensqualität der Patienten aus. Darüber hinaus zeigt TEM zugleich kürzere Operationszeiten, postoperative stationäre Aufenthalte und damit Therapiekosten.

Für den erfolgreichen Einsatz dieses Operationsverfahrens ist nach aktuellem Wissenstand eine strenge präoperative Patientenselektion und enge Indikationsstellung unabdingbar. Nach derzeitiger Datenlage sind dies ausschließlich benigne Tumore wie Adenome sowie die Carcinomata in situ und low-risk-pT1Karzinome. Die hier dargelegten Frühergebnisse für TEM deuten zudem auf Vorteile durch den Einsatz der Ultraschalldissektionstechnologie im Vergleich zur konventionellen Hochfrequenzstromdissektion hin.

Aufgrund des noch kurzen Nachbeobachtungszeitraumes und der begrenzten Fallzahl sind abschließende Aussagen allerdings noch nicht möglich. Hierfür sind Langzeitergebnisse im Rahmen prospektiver randomisierter Studien erforderlich, die aufgrund der niedrigen Inzidenz dieser seltenen Präkanzerosen und Frühkarzinome des Rektums übergreifend an Multicenterstudien erhoben werden müssen. 


\section{$6 \quad$ Literatur}

Amaral JF (1994):

Ultrasonic dissection

End Surg, 2, 181-185

Ambacher T, Kasperk R, Schumpelick V (1999):

Einfluß der transanalen Exzision auf die Rezidivrate beim Stadium-I-Rectumcarcinom im Vergleich zu radikal resezierenden Verfahren

Chirurg, $\underline{70}, 1469-1474$

\section{Arminski CT, McLeand DW (1964):}

Incidence and distribution of ad. Polyp of the colorectum. 1000 autopsy examinations Dis Colon Rectum, $\underline{7}, 249$

Arribas del Amo D, Ramirez Rodriguez JM, Aguilella Diago V, Elia Guedea M, Palacios Fanlo MJ, Martinez Diez M (2000):

Transanal endoscopic surgery for rectal tumors

Rev Esp Enferm Dig, 82, 526-535

Azimuddin K, Riether RD, Stasik JJ, Rosen L, Khubchandani IT, Reed JF (2000):

Transanal endoscopic microsurgery for excision of rectal lesions: technique and intial results

Surg Laparosc Endosc Percutan Tech, 10, 382-378

Banerjee AK, Jehle EC, Kreis ME, Schott UG, Claussen CD, Becker HD, Starlinger M, Buess GF (1996):

Prospective study of the proctographic and functional consequences of transanal endoscopic microsurgery

Br J Surg, $\underline{83}, 211-213$ 


\section{Batignani G, Monaci I, Ficari F, Tonelli F (1991):}

What effects contingence after anterior resection of the rectum?

Dis Colon Rectum, 34, 329-335

Benoist S, Taffinder N, Gould S, Ziprin P, Chang A, Darzi A (2001):

Transanal endoscopic microsurgery: a forgotten minimally invasive technique Gastroenterol Clin Biol, 25, 369-374

Beynon J, Mortensen NJ McC, Foy JL, Virjee J, Goddard P (1986):

Endorectal sonography: laboratory and clinical experience in Bristol Int J Colorect Dis, 1, 212-215

Blair S, Ellenhorn JD (2000):

Transanal excision for low rectal cancers is curative in early-stage disease with favourable histology

Am Surg, $\underline{66}, 817-820$

Bleday R (1997):

Local excision of rectal cancer

World J Surg, 21, 706-714

Blumberg D, Paty PB, Guilem JG, Picon Al, Minsky BD, Wong WD, Cohen AM (1999):

All patients with small intramural rectal cancers are at risk for lymph node metastasis

Dis Colon Rectum, $\underline{42}, 881-885$

Boland CR (1993):

The biology of colorectal cancer

Cancer, $\underline{71}$, 4180-4186 
Bonnen M, Crane C, Vauthey JN, Skibber J, Delclos ME, Rodrigues-Bigas M, Hoff PM, Lin E, Eng C, Wong A, Janjan NA, Feig BW (2004):

Long-term results using local excision after preoperative chempradiation among selected $\mathrm{T} 3$ rectal cancer patients

Int J Radiat Oncol Biol Phys, $\underline{60}, 1098-1105$

Bouvet M, Mlias M, Giacco GG et al. (1999):

Predictors of recurrence after local excision and postoperative chemoradiation therapy of adenocarcinma of the rectum

Ann Surg Oncol, $\underline{6}, 26-32$

Brodsky JT, Richard GK, Cohen AM, Minsky BD (1992):

Variables correlated with the risk of lymph node metastasis in early rectal cancer

Cancer, $\underline{69}, 322-326$

Buess G, Kayser J (1996):

Technik und Indikation zur sphinktererhaltenden transanalen Resektion ebim Rektumcarzinom

Chirurg, $\underline{67}, 121-128$

Buess G, Theiss R, Hutterer F, Pichlmaier H, Pelz C, Holfeld T, Said S, Isselhard W (1983):

Transanal endoscopic surgery of the rectum - testing a new method in animal experiments

Leber Magen Darm, $\underline{13}, 73-77$

Buess G, Kipfmuller K, Hack D, Grüßner R, Heintz A, Junginger T (1988a):

Technique of transanal endoscopic microsurgery

Surg Endosc, 2 2, 71-75 
Buess G, Kipfmüller K, Ibald R, Heintz A, Hack D, Braunstein S, Gabbert H, Junginger T (1988b):

Clinical results of transanal endoscopic microsurgery

Surg Endosc, 2, 245-250

Carmona JA, Oritz H, Preez-Cabanas I (1991):

Alterations in the anorectal function after anterior resection for cancer of the rectum Int J Colorectal Dis, $\underline{6}, 108-110$

Chakravarti A, Compton CC, Shellito PC, Wood WC, Landry J, Machuta SR, Kaufman D, Ancukiewicz M, Willett CG (1999):

Long-term follow-up of patients with rectal cancer managed by local excision with and without adjuvant irradiation

Ann Surg, 230, 49-54

Chorost MI, Petrelli NJ, McKenna M, Kraybill WG, Rodriguez-Bigas MA (2001):

Local excision of rectal cancer

Am Surg, $\underline{67}, 774-779$

Cocilovo C, Smith LE, Stahl T, Douglas J (2003):

Transanal endoscopic excision of rectal adenomas

Surg Endosc, 17, 1461-1463

Czerny V (1880):

Zur Darmresektion

Berl Klin Wochenschr, 17, 637

Demartines N, von Flue MO, Harder FH (2001):

Transanal endoscopic microsurgical excision of rectal tumors: indications and results World J Surg, 25, 870-875 


\section{Dixon CF (1958):}

Anterior resection for malignant tumours of the upper part of the rectum and lower part of the sigmoid

Trans Am Surg Assoc, $\underline{66}$, 175-180

Farmer KC, Wale R, Winnett J, Cunningham I, Grossberg P, Polglase A (2002):

Transanal endoscopic microsurgery: the first 50 cases

ANZ J Surg, $\underline{72}$, 854-856

Garcia-Aguilar J (2001):

Colorectal cancer experiment meodels

Rev Esp Enferm Dig, 93, 398-401

Havenga K, Huang Y, Enker WE, Welvaart K, De Roy van Zuidewijn DB, Cohen AM (1996):

Aggressive versus conventional strategies in the treatment of rectal adenocarcinoma Surg Oncol, $\underline{5}, 183-188$

Heald RJ, Moran BJ, Ryall RD, Sexton R, MacFarlane JK (1998):

Rectal cancer: the Basingstoke experience of total mesorectal excision Arch Surg, 133, 894-899

Heintz A, Mörschel M, Junginger T (1997):

Transanale endoskopische Operationen bei benignen Rektumpolypen

Coloproctology, $\underline{19}, 175-178$

Heintz A, Mörschel M, Junginger T (1998):

Comparison of results after transanal endoscopic microsurgery and radical resection for T1 carcinoma of the rectum

Surg Endosc, 12, 1145-1148 
Hermanek P, Gall FP (1986):

Early (microinvasive) colorectal carcinoma

Int J Colorect Dis, 1, 79-84

Hermanek P, Marzoli GP:

Lokale Therapie des Rektumskarzinoms. Verfahren in kurativer Intention

Springer, Berlin 1994

Hermanek P, Hutter RVP, Sobin LH, Wagner G, Wittekind C:

TNM Atlas. Illustrated guide to the TNM / pTNM Classification of malignant tumours 4th edn, 2nd rev, Springer, Berlin Heidelberg New York 1999

Hildebrandt U, Feifel G (1985):

Prooperative staging of rectal cancer by intrarectal ultrasound

Dis Colon Rectum, 28, 42-46

Hildebrandt U, Schuder G, Feifel G (1994):

Prooperative staging of rectal and colonic cancer

Endoscopy, 26, $810-812$

Hochenegg J (1900):

Meine Operationserfolge bei Rectumcarcinom

Wien Klin Wochenschr, 13, 399

Jørgensen OD, Kronborg, Fenger C (1993):

The funen abdomen follow-up study. Characteristics of patients and initial adenomas in relation to severe dysplasia

Scand J Gastroenterol, 28, 239-243

\section{Junginger T:}

Rektumkarzinom

Deutsche Krebsgesellschaft: Kurzgefasste Interdisziplinäre Leitlinien 2004, 3. Auflage, Zuckschwerdt Verlag, München Bern Wien New York 2004 
Kanaya S, Komori J, Sarumaru S, Matsushita T, Isoba H, Katayama T, Wada Y, Otoshi M (1998):

Use of hamonic scalpel for trananal endoscopic microsurgery

Surg Endosc, 12, 711

Kasperk R, Willis S, Riesener KP, Schumpelick V (2001):

Evidence-based surgery of rectal carcinoma

Zentralbl Chir, 126, 295-301

Katti G (2004):

An evaluation of transanal endoscopic surgery for rectal adenoma and caricnoma JSLS, 8, 123-126

Kessler H, Hermanek P Jr, Wiebelt H (1993):

Operative mortality in carcinoma of the rectum. Resuults of the german multicentre study

Int J Colorect Dis, $\underline{8}, 158-166$

Kim CJ, Yeatman TJ, Coppola D, Trotti A, Williams B, Barthel JS, Dinwoodie W, Karl RC, Marcet J (2001):

Local excision of T2 and T3 rectal cancer after downstaging chemoradiation Ann Surg, 234, 352-358

Kinn A-C, Öhman U (1986):

Bladder and sexual function after surery for rectal cancer

Dis Colon Rectum, 29, 43-48

Kraemer M, Wiratkapun S, Seow-Choen F, Ho YH, Eu KW, Nyam D (2001):

Stratifying risk factors for follow-up: a comparison of recurrent and nonrecurrent colorectal cancer

Dis Colon Rectum, 44, 815-821 
Kraemer M, Wiratkapun S, Seow-Choen F, Ho YH, Eu KW, Nyam D (2001):

Stratifying risk factors for follow-up: a comparison of recurrent and nonrecurrent colorectal cancer

Dis Colon Rectum, $\underline{44}, 815-821$

\section{Kraske P (1887):}

Die sacrale Methode der Exstirpation von Mastadarmkrebsen und die Resectio recti Berl Klin Wochenschr, 24

Kunihiro M, Tanaka S, Haruma K, Kitadai Y, Yoshihara M, Sumii K, Kajiyama G, Nishiyama M (2000):

Electrocautery snare resection stimulates cellular proliferation of residual colorectal tumor

Dis Colon Rectum, $\underline{43}, 1107-1115$

Lamont JP, McCarty TM, Digan RD, Jacobson R, Tulanon P, Lichliter WE (2000):

Should locally excised T1 rectal cancer receive adjuvant chemoradiation?

Am J Surg, $\underline{180}$, 402-405

\section{Langer C, Liersch T, Wüstner M, Muller D, Kilian D, Fuzesi L, Becker H (2001a):}

Endosonographie bei epithelialen Rektumtumoren - Stellenwert in einem differenzierten Therapiekonzept -

Chirurg, $\underline{72}, 266-271$

Langer C, Markus P, Liersch T, Fuzesi L, Becker H (2001b):

UltraCision or high-frequency knife in transanal endoscopic microsurgery (TEM)? Advantages of a new procedure

Surg Endosc, 15, 513-517 


\section{Lezoche, Guerrieri M, Paganini AM, Feliciotti F (2002):}

Long-term results of patients with pT2 rectal cancer treated with radiotherapy and transanal endoscopic microsurgical excision

World J Surg, 26, 1170-1174

\section{Lirici MM, Di Paola M, Ponzano C, Huscher CG (2003):}

Combining ultrasonic dissection and the Storz operation rectoscope

Surg Endos, 17, 1292-1297

\section{Lisfranc P (1826):}

Mémoire sur l'excision de la partie inférieure du rectum devenue carcinomateuse

Rev Med Franc, 2, 380

Lloyd GM, Sutton CD, Marshall LJ, Baragwanath P, Jameson JS, Scott AD (2002):

Transanal endoscopic microsurgery - lesson form a single UK centre series

Colorectal Dis, $\underline{6}, 467-472$

Maeda K, Maruta M, Dato H, Hanai T, Masumori K, Matumoto M, Koide Y, Matuoka H, Katuno H (2004):

Outcomes of novel transanal operation for selected tumors of the rectum

J Am Coll Surg, 199, 353-360

Mainprize KS, Mortensen NJ, Warren BF (1998):

Early colorectal cancer: recognition, classification and treatment

Br J Surg, $\underline{85}, 469-476$

Mandel JS, Church TR, Ederer F, Bond JH (1999):

Colorectal cancer mortality: effectiveness of biennial screening for fecal occult blood J Natl Cancer Inst, $\underline{91}$, 434-437 
Manfredi S, Benhamiche AM, Meny B, Cheynel N, Rat P, Faivre J (2001):

Population-based study of factors influencing occurence and prognosis of local recurrence after surgery for rectal cancer

Br J Surg, 요, 1221-1227

\section{Mason AY (1976):}

Rectal cancer: the spectrum of selective surgery

Proc R Soc Med, $\underline{69}, 237$

\section{Matzel KE, Merkel S, Hohenberg W (2003):}

Lokale Therapieprinzipien beim Rektumkarzinom

Chirurg, $\underline{74}$, 897-904

Matzel KE, Kamm MA, Stosser M, Baeten CG, Christiansen J, Madoff R, Mellgren A, Nicholls RJ, Rius J, Rosen H (2004):

Sacral spinal nerve stimulation for faecal incontinence: multicentre study Lancet $\underline{363}, 1270-1276$,

McDermott FT, Huges ESR, Pihl E, Johnson WR, Price AB (1985):

Local recurrence after potentially curative resection for retal cancer in a series of 1008 patients

Br J Surg, $\underline{72}, 34-37$

Mellgren A, Sirivongs P, Rothenberger DA, Madoff RD, García-Aguilar J (2000):

Is local excision adequate therapy for early rectal cancer?

Dis Colon Rectum, 느, 1064-1071

\section{Meng WC, Lau PY, Yip AW (2004):}

Treatment of early rectal tumours by transanal endoscopic microsurgery in Hong Kong: prospective study

Hong Kong Med J, 10, 239-243 
Mentges B, Buess G, Effinger G, Manncke K, Becker HD (1997):

Indications and results of local treatment of rectal cancer

Br J Surg, $\underline{84}$, 348-351

Midgley R, Kerr D (1999):

Colorectal cancer

Lancet, $\underline{353}$, 391-399

Miles WE (1908):

A method to performing abdominoperineal excision for carcinoma of the rectum and of the terminal portion of the pelvic colon

Lancet, 1908, 2, 1812-1813

Mörschel M, Heintz A, Bußmann M, Junginger T (1998):

Follow-up after transanal endoscopic microsurgery or transanal excision of large benign rectal polyps

Langenbeck's Arch Surg, $\underline{383}, 320-324$

Mörschel M, Heintz A, Junginger T (1999):

Das Entartungsrisiko als benigne eingestufter breitbasig aufsitzender Rektumpolypen

Zentralbl Chir, 124, 226-229

Morson BC (1985):

Histological criteria for local excision

Br J Surg, Suppl. September 1985, 53-58

Muto T, Bussey HJ, Morson BC (1975):

The evolution of cancer of the colon and rectum

Cancer, $\underline{36}, 2251-2270$

Nascimbeni R, Burgart LJ, Nivatvongs S, Larson DR (2002):

Risk of lymph node metastasis in T1 carcinoma of the colon and rectum

Dis Colon Rectum, 45, 200-206 
Neary P, Makin GB, White TJ, White E, Hartley J, MacDonald A, Lee PW, Monson JR (2003):

Transanal endoscopic microsurgery: a viable operative alternative in selected patients with rectal lesions

Ann Surg Oncol, 10, 1106-1111

\section{Nöthinger F, Birrer S:}

Chirurgie

In: Berchtold R, Hamelmann H, Peiper H-J, Trentz O (Eds.), Urban und Fischer, München 1999, 553-569

O`Brien MJ, Winawer SJ, Zauber AG (1990):

The national polyp study: patient and polyp characteristics associated with highgrade dyplasia in colorectal carcinoma

Gastroenterology, $\underline{98}, 371-379$

Palma P, Freudenberg S, Samel S, Post S (2004):

Transanal endoscopic microsurgery: indications and results after 100 cases

Colorectal Dis, $\underline{6}, 350-355$

Parks AG (1970):

A technique for the removal of large villous tumours in the rectum

Proc R Soc Med, 63 Suppl, 89-91

Paty PB, Nash GM, Baron P, Zakowski M, Minsky BD, Blumberg D, Nathanson DR, Guillem JG, Enker WE, Cohen AM, Wong WD (2002):

Long-term results of local excision for rectal cancer

Ann Surg, 236, 522-529

Platell C, Denholm E, Makin G (2004):

Efficacy of transanal endoscopic microsurgery in the management of rectl polyps J Gastroenterol Hepatol, 19, 767-772 


\section{Renner K, Rosen HR, Novi G, Holbing N, Schiessel R (1999):}

Quality of life after surgery for rectal cancer: do we still need a permanent colostomy?

Dis Colon Rectum, 42, 1160-1167

\section{Reybeard JF (1843):}

Mémoire sur une tumeur cancéreuse affectant l'iliaqeu de côlon; ablation de la tumeur et de l'intestin; réunion directe et immédiate des deux bouts de cet organe Bull Acad de Med, $\underline{9}, 1031$

Richter M, Herzog U, Lang C, Schuppisser JP, Ackermann C, Tondelli P (1996):

Die Behandlung des Rektumadenoms. Retrospektive Untersuchung stationär behandelter Patienten

Schweiz Med Wochenschr Suppl, 79, 80S-84S

\section{Robert-Koch-Institut:}

Krebs in Deutschland. Häufigkeiten und Trends

2. Auflage, Arbeitsgemeinschaft bevölkerungsbezogener Krebsregister in Deutschland, Robert-Koch-Institut (Eds.), Saarbrücken 1999

\section{Romeis B:}

Mikroskopische Technik

In: Böck P (ed) 17. Auflage, Urban-Schwarzenberg, München Wien Baltimore 1989, 219-263

Ruo L, Tickoo S, Klimstra DS, Minsky BD, Saltz L, Mezumdar M, Paty PB, Wong WD, Larson SM, Cohen AM, Guillem JG (2002):

Long-term prognostic significance of rectal cancer response to preoperative radiation and chemotherapy

Ann Surg, 236, 75-81

\section{Saclarides TJ (1997):}

Transanal endoscopic microsurgery

Surg Clin North Am, $\underline{77}, 229-239$ 
Scalarides TJ (2004):

Transanal endoscopic microsurgery

Semin Laparosc Surg, 11, 45-51

Sakamoto GD, MacKeigan JM, Senagore AJ (1991):

Transanal excision of large rectal villous adenomas

Dis Colon Rectum, $\underline{34}$, 880-885

Salm R, Lampe H, Bustos A, Matern U (1994):

Experience with TEM in Germany

Endosc Surg Allied Technol, 2, 251-254

Sauer R, Becker H, Hohenberger W, Rodel C, Wittekind C, Fietkau R, Martus P, Tschmelitsch J, Hager E, Hess CF, Karstens JH, Liersch T, Schmidberger H, Raab R (2004):

Preoperative versus postoperative chemoradiotherapy for rectal cancer

N Eng J Med, $\underline{351}, 1731-1740$

Schäfer H, Baldus SE, Gasper F, Hölscher AH (2004):

Transanale endoskopische Mikrochirurgie beim pT1-Low-risk-Rektumkarzinom

Zusammenhang zwischen Submukosainfiltrationstiefe und Lokalrezidivrate

Chirurg, Epub, Online First, DOI 10.1007/s00104-004-0929-2

Schmiegel W, Pox C, Adler G, Fleig W, Folsch UR, Fruhmorgen P, Graeven U, Hohenberger W, Holstege A, Junginger T, Kuhlbacher T, Porschen R, Propping P, Riemann JF, Sauer R, Sauerbruch T, Schmoll HJ, Zeitz M, Selbmann HK (2004):

Kolorektales Karzinom: Prävention und Früherkennung in der asymptomatischen Bevölkerung - Vorsorge bei Risikogruppen - Endoskopische Diagnostik und Therapie von Polyen und Karzinomen

Z Gastroenterol, $\underline{42}, 1129-1177$ 
Schön D, Bertz J, Görsch B, Haberland J, Ziegler H, Stegmaier C, Eisinger B, Stabenow R:

Gesundheitsberichterstattung für Deutschland Schwerpunktbericht Entwicklung der Überlebensraten von Krebspatienten in Deutschland

Verlag Robert Koch-Institut, Berlin 1999

Sengupta S, Tjandra JJ (2001):

Local excision of rectal cancer:What is the evidence?

Dis Colon Rectum, $\underline{44}, 1345-1361$

Steele RJC, Hershman MJ, Mortensen NJ, Armitage NCM, Scholefield JH (1996):

Transanal endoscopic microsurgery - initial experience from three centres in the United Kingdom

Br J Surg, 주, 207-210

Stipa F, Lucandri G, Ferri M, Casula G, Ziparo V (2004):

Local excision of rectal cancer with transanal endoscopic microsurgery (TEM)

Anticancer Res, 24, 1167-1172

Stipa S, Stipa F, Lucandri G, Ziparo V (2000):

Surgery of rectal tumors

Przegl Lek, 57, 66-68

Taylor RH, Hay JH, Larsson SN (1998):

Transanal local excision of selected low rectal tumors

Am J Surg, 175, 360-363

Visser BC, Varma MG, Welton ML (2001):

Local therapy of rectal cancer

Surg Oncol, 10, 61-69 
Vogelstein B, Kinzler KW:

The Genetic Basis of Human Cancer

2. Auflage, McGraw-Hill-Health Professions Division, Maidenhead, United Kindom 2002

Weir RT (1901):

An improved method of treating high seated cancer of the rectum

JAMA, $\underline{37}, 801$

Winde G, Nottberg H, Keller R, Schmid KW, Bünte H (1996a):

Surgical care for early rectal carcinomas (T1). Transanal endoscopic microsurgery vs. anterior resection

Dis Colon Rectum, $\underline{39}$, 969-976

Winde G, Schmid KW, Reers B, Bünte H (1996b):

Mikrochirurgie (TEM) im prospektiven Vergleich zu konventioneller transanaler Abtragung oder anteriorer Rektumresektion bei Adenomen und oberflächlichen Karzinomen

Langenbecks Arch Chir Suppl Kongressbd, 113, 265-268 


\section{Danksagung}

Mein besonderer Dank gilt PD Dr. C. Langer, unter dessen freundschaftlicher und kompetenter Betreuung die Arbeit überhaupt nur möglich wurde.

Für die Überlassung dieses überaus interessanten Themas möchte ich mich herzlich bei Prof. Dr. H. Becker bedanken.

Auch Dr. A. Schaper gebührt meine Dankbarkeit, da er mich zur Durchführung dieser Arbeit ermutigte.

Darüber hinaus möchte ich mich für die gute Zusammenarbeit mit der Abteilung Medizinische Statistik und der Abteilung Genetische Epidemiologie, hier insbesondere Frau B. Geiling und Dr. A. Siemer, bedanken, deren Beratung zum Gelingen dieser Arbeit maßgeblich beigetragen hat.

Zu guter Letzt möchte ich mich bei meinen Eltern bedanken, ohne deren ständige Bereitschaft zur Diskussion der Inhalte meiner Arbeit diese Dissertation wahrscheinlich nicht wäre beendet worden. 


\section{Lebenslauf}

Ich wurde am 17.01.1975 als dritter Sohn von Anke und Torsten Süß in Oldenburg geboren. Im Zeitraum zwischen 1981 und 1985 besuchte ich die Grundschule Ohmstede (Oldenburg). Die weiterführende Ausbildung erfolgte in den Jahren zwischen 1985 und 1987 an der Orientierungsstufe Flötenteich (Oldenburg). Nach Besuch des Gymnasiums Cäcilienschule (Oldenburg) in den Jahren 1987 bis 1994 erlangte ich am 14.6.1994 die allgemeine Hochschulreife.

Im Zeitraum zwischen 1994 und 1995 leistete ich den Wehrdienst bei der Luftlandesanitätskompanie 270 (Varel) ab.

Ab Oktober 1995 betrieb ich das Studium der Mathematik und der Sportwissenschaften für Lehramt an Gymnasien an der Georg-August-Universität zu Göttingen. Im Oktober des Jahres 1997 erfolgte eine Umstellung der Studienfächer auf den Studiengang Sportwissenschaften unter Hinzunahme des Studiums der Humanmedizin. Das Grundstudium der Humanmedizin beendete ich am 22.2.1999. Am 13.4.2000 legte ich die erste Staatsprüfung, am 18.9.2002 die zweite Staatsprüfung und am 19.11.2003 die dritte Staatsprüfung der Humanmedizin jeweils an der Georg-August-Universität zu Göttingen ab.

Seit dem 1.1.2004 war ich als Arzt im Praktikum in der chirurgischen Abteilung, und fortlaufend ab dem 1.10.2004 als Assistenzarzt in Weiterbildung in der unfallchirurgischen Abteilung der Mittelweser Kliniken $\mathrm{GmbH}$ beschäftigt. 